\title{
Non-perturbative definition of the QCD energy-momentum tensor on the lattice
}

\author{
Mattia Dalla Brida, ${ }^{a, b}$ Leonardo Giusti ${ }^{a, b}$ and Michele Pepe ${ }^{b}$ \\ ${ }^{a}$ Dipartimento di Fisica, Università di Milano-Bicocca, \\ Piazza della Scienza 3, I-20126 Milano, Italy \\ ${ }^{b}$ INFN, Sezione di Milano-Bicocca, \\ Piazza della Scienza 3, I-20126 Milano, Italy \\ E-mail: mattia.dallabrida@unimib.it, leonardo.giusti@mib.infn.it, \\ pepe@mib.infn.it
}

ABSTRACT: We present a strategy to define non-perturbatively the energy-momentum tensor in Quantum Chromodynamics (QCD) which satisfies the appropriate Ward identities and has the right trace anomaly. The tensor is defined by regularizing the theory on a lattice, and by fixing its renormalization constants non-perturbatively by suitable Ward identities associated to the Poincare invariance of the continuum theory. The latter are derived in thermal QCD with a non-zero imaginary chemical potential formulated in a moving reference frame. A renormalization group analysis leads to simple renormalizationgroup-invariant definitions of the gluonic and fermionic contributions to either the singlet or the non-singlet components of the tensor, and therefore of their form factors among physical states. The lattice discussion focuses on the Wilson discretization of quark fields but the strategy is general. Specific to that case, we also carry out the analysis for the on-shell $\mathrm{O}(a)$-improvement of the energy-momentum tensor. The renormalization and improvement programs profit from the fact that, as shown here, the thermal theory enjoys de-facto automatic $\mathrm{O}(a)$-improvement at finite temperature. The validity of the proposal is scrutinized analytically by a study to 1-loop order in lattice perturbation theory with shifted and twisted (for quarks only) boundary conditions. The latter provides also additional useful insight for a precise non-perturbative calculation of the renormalization constants. The strategy proposed here is accessible to Monte Carlo computations, and in this sense it provides a practical way to define non-perturbatively the energy-momentum tensor in QCD.

KEYworDS: Lattice Quantum Field Theory, Nonperturbative Effects

ARXIV EPRINT: 2002.06897 


\section{Contents}

1 Introduction 1

2 The energy-momentum tensor in the continuum 3

2.1 Thermal QCD in the presence of shift and imaginary chemical potential 3

2.2 Finiteness of $T_{\mu \nu}$ and trace anomaly 5

2.2.1 The non-singlet 5

2.2.2 The singlet $\quad 7$

$\begin{array}{llr}2.3 & \text { Finite volume } & 9\end{array}$

3 The energy-momentum tensor on the lattice 10

$\begin{array}{lll}3.1 & \text { Non-perturbative renormalization conditions } & 12\end{array}$

$\begin{array}{lll}4 & \mathrm{O}(a) \text {-improvement } & 13\end{array}$

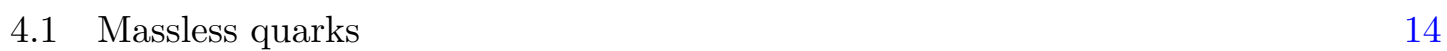

$\begin{array}{lll}4.2 & \text { Mass-degenerate quarks } & 14\end{array}$

$\begin{array}{lll}4.3 \text { Mass non-degenerate quarks } & 15\end{array}$

$\begin{array}{lll}4.4 & \text { Improvement conditions } & 15\end{array}$

5 Perturbative analysis $\quad 16$

$\begin{array}{lll}5.1 & \text { Free-energy density } & 17\end{array}$

$\begin{array}{ll}5.2 \text { Energy-momentum tensor } & 17\end{array}$

$\begin{array}{lll}\text { 5.2.1 Renormalization constants of the sextet } & 19\end{array}$

5.2.2 Renormalization constants of the triplet 20

$\begin{array}{lll}5.2 .3 & \text { Improvement coefficients } & 21\end{array}$

6 Conclusions 23

$\begin{array}{ll}\text { A Conventions and useful identities } & 24\end{array}$

B Continuum theory $\quad 25$

B.1 Dimensional regularization 26

$\begin{array}{ll}\text { C Lattice theory } & 27\end{array}$

$\begin{array}{ll}\text { D Automatic } \mathrm{O}(a) \text {-improvement with massless quarks } & 28\end{array}$

$\begin{array}{ll}\text { E Propagators and vertices for perturbation theory } & 29\end{array}$

E.1 Gluon propagator $\quad 30$

E.2 Ghost propagator 31

E.3 Fermion propagator 31

E.4 Gluonic interaction 31

E.5 Ghost-gluon interaction 32 
F The free energy density 33

F.1 $\mathrm{O}(a)$-improved action 34

G Expectation value of the vector current $\quad 35$

G.1 $\mathrm{O}(a)$-improved action 36

H Expectation values of sextet components of $\boldsymbol{T}_{\mu \nu} \quad 37$

H.1 $\mathrm{O}(a)$-improved action $\quad 40$

I Expectation values of triplet component of $T_{\mu \nu} \quad 42$

$\begin{array}{lll}\text { I.1 } & \mathrm{O}(a) \text { improvement } & 42\end{array}$

$\begin{array}{lll}\text { J Integrals } & 43\end{array}$

\section{Introduction}

The energy-momentum tensor, $T_{\mu \nu}$, is a central quantity in a quantum field theory since it groups together the currents associated to the invariance of the theory under spacetime translations, from which also those associated to the larger Poincaré group and scale invariance can be built. Apart from the theoretical one, the great interest in the energymomentum tensor is manifold. For instance, in general relativity it enters the Einstein field equations acting as the source of space-time curvature generated by the fields. In thermal field theories its expectation values provide the equation of state of the theory, while its twopoint correlation functions allow one to measure the transport coefficients of the plasma.

The only known non-perturbative regularization of QCD is the lattice where, however, the Poincaré group is explicitly broken into discrete subgroups, and the full symmetry is recovered in the continuum limit. As a consequence, a given definition of the energymomentum tensor on the lattice needs to be properly renormalized to guarantee that the associated charges are the generators of the Poincaré group in the continuum limit, and that the trace anomaly is correctly reproduced.

In order to construct the renormalized energy-momentum tensor, the way to proceed is to impose suitable Ward Identities (WIs) at fixed lattice spacing that hold up to cutoff effects which vanish in the continuum limit. Indeed that problem was addressed in refs. $[1$, 2] for the Yang-Mills theory and QCD, where it was shown that on the lattice the 10dimensional symmetric tensor $T_{\mu \nu}$ breaks into the sum of a sextet, a triplet and a singlet representation of the hypercubic group. Each one of those three parts picks up finite renormalization constants which were computed to 1-loop order in perturbation theory [35], see also [6-8]. However, it was not clear how to define the renormalization constants so that they could be computed non-perturbatively. 
An important step forward was made a few years ago by noticing that useful WIs to fix the renormalization constants are obtained by considering the theory in a finite box, where the Euclidean Lorentz symmetry is also softly broken by its shape [9-11]. In particular, if the length in one (temporal) direction $L_{0}$ is chosen to be finite (thermal theory), interesting WIs follows. They become particularly simple and of practical use if the periodicity axes are tilted with respect to the lattice grid (moving reference frame), i.e. if the hard breaking of the Poincaré symmetry due to the lattice discretization and the soft one due to the finite temporal direction are not aligned. This set-up has a natural implementation in the Euclidean path-integral formulation in terms of shifted boundary conditions [9-12]. These ideas led for the first time to a non-perturbative definition of $T_{\mu \nu}$ in the SU(3) Yang-Mills theory [13].

The purpose of this paper is to present the WIs for defining non-perturbatively the energy-momentum tensor in QCD. By generalizing what has been done in the SU(3) YangMills theory, we work in the framework of shifted boundary conditions supplemented by the presence of an imaginary chemical potential. It is the latter that gives us the handle to solve the problem of the mixing between the gluonic and fermionic parts of the tensor since the chemical potential couples differently to quark and gluons. Most interestingly, the derived relations can be used in practice to carry out the non-perturbative numerical computation of the renormalization constants of $T_{\mu \nu}[14]$.

Although the strategy is general, for definiteness we consider the Wilson formulation of quarks on the lattice with and without the Sheikholeslami-Wohlert improving term [15]. In the presence of the latter, we also carry out the analysis for the on-shell $\mathrm{O}(a)$-improvement of the energy-momentum tensor field. The implementation of the renormalization and of the improvement program turn out to be greatly simplified because the thermal theory enjoys de-facto automatic $\mathrm{O}(a)$-improvement at finite temperature.

The validity of the proposal is scrutinized analytically to 1-loop order in lattice perturbation theory with shifted and twisted (for quarks only) boundary conditions. The results obtained this way serve also to give a 1-loop improved definition of the non-perturbative renormalization constants with the aim of reducing discretization effects. For completeness, we notice that over the last few years alternative methods, based on the Yang-Mills gradient flow [16], have also been explored for renormalizing non-perturbatively the energymomentum tensor [17-22].

The paper is organized as follows. In section 2 we derive the basic identities which are later enforced on the lattice to fix the renormalization constants of the energy-momentum tensor, and we construct the renormalization-group-invariant (RGI) definitions of its gluonic and fermionic components. In the following section we show how these relations define non-perturbatively the energy-momentum tensor on the lattice, while in section 4 we discuss the $\mathrm{O}(a)$ improvement. In the following section we investigate the renormalization conditions in perturbation theory, and we compute the renormalization constants and the improvement coefficients of the gluonic and fermionic parts of the energy-momentum tensor to 1-loop order. Section 6 contains our conclusions. Notations, conventions, and technical details are reported in several appendices. 


\section{The energy-momentum tensor in the continuum}

In this section we consider QCD in the continuum, for definitions and conventions see appendices $\mathrm{A}$ and $\mathrm{B}$. We are interested in correlation functions of the energy-momentum tensor $T_{\mu \nu}$ with gauge-invariant operators inserted at a physical distance. As reviewed in [4, 13], it is appropriate in those cases to consider the symmetric gauge-invariant definition of the energy-momentum tensor given by

$$
T_{\mu \nu}=T_{\mu \nu}^{G}+T_{\mu \nu}^{F}
$$

where the first term is the gluonic component ${ }^{1}$

$$
T_{\mu \nu}^{G}=\frac{1}{g_{0}^{2}}\left\{F_{\mu \alpha}^{a} F_{\nu \alpha}^{a}-\frac{1}{4} \delta_{\mu \nu} F_{\alpha \beta}^{a} F_{\alpha \beta}^{a}\right\},
$$

while the second is the fermionic one given by

$$
T_{\mu \nu}^{F}=\frac{1}{4}\left\{\bar{\psi} \gamma_{\mu} \overleftrightarrow{D}_{\nu} \psi+\bar{\psi} \gamma_{\nu} \overleftrightarrow{D}_{\mu} \psi\right\}-\frac{1}{4} \delta_{\mu \nu} \bar{\psi}\left\{\frac{1}{2} \gamma_{\alpha} \overleftrightarrow{D}_{\alpha}+M_{0}\right\} \psi
$$

where $\overleftrightarrow{D}_{\mu}$ is defined in eq. (B.10).

\subsection{Thermal QCD in the presence of shift and imaginary chemical potential}

For our strategy it is instrumental to consider the thermal theory with a non-zero imaginary chemical potential formulated in a moving reference frame. Its grand canonical partition function reads

$$
\mathcal{Z}\left(L_{0}, \boldsymbol{\xi}, \mu_{\mathcal{I}}\right)=\operatorname{Tr}\left\{e^{-L_{0}\left(\widehat{H}-i \boldsymbol{\xi} \cdot \widehat{\boldsymbol{P}}-i \mu_{\mathcal{I}} \widehat{N}\right)}\right\},
$$

where the trace is over all the states of the Hilbert space, $L_{0}$ is the finite length of the temporal direction, and $\widehat{N}$ is the quark number operator (three times the baryon number). The Hamiltonian $\widehat{H}$, the total momentum operator $\widehat{\boldsymbol{P}}$, and the imaginary chemical potential $\mu_{\mathcal{I}}$ are expressed in a reference frame where the system is moving at a velocity $\boldsymbol{v}$ which, by analytic continuation, we fix to the imaginary value $\boldsymbol{v}=i \boldsymbol{\xi}$.

In the Euclidean path-integral formalism the partition function (2.4) is given by

$$
\mathcal{Z}\left(L_{0}, \boldsymbol{\xi}, \mu_{\mathcal{I}}\right)=\left.Z\left(L_{0}, \boldsymbol{\xi}, \theta_{0}\right)\right|_{\theta_{0}=-L_{0} \mu_{\mathcal{I}}},
$$

where $Z\left(L_{0}, \boldsymbol{\xi}, \theta_{0}\right)$ is the ordinary QCD path integral as defined in eq. (B.1) with fields which, in the time direction, satisfy periodic boundary conditions up to a shift $L_{0} \boldsymbol{\xi}[9-11]$ and a twist of the fermion fields [23]. Specifically, the gauge field $A_{\mu}$ obeys

$$
A_{\mu}\left(x_{0}+L_{0}, \boldsymbol{x}\right)=A_{\mu}\left(x_{0}, \boldsymbol{x}-L_{0} \boldsymbol{\xi}\right),
$$

while the fermion fields, on top of the usual minus sign, pick up also a non-trivial twist phase at the boundaries so that

$$
\begin{aligned}
& \psi\left(x_{0}+L_{0}, \boldsymbol{x}\right)=-e^{i \theta_{0}} \psi\left(x_{0}, \boldsymbol{x}-L_{0} \boldsymbol{\xi}\right), \\
& \bar{\psi}\left(x_{0}+L_{0}, \boldsymbol{x}\right)=-e^{-i \theta_{0}} \bar{\psi}\left(x_{0}, \boldsymbol{x}-L_{0} \boldsymbol{\xi}\right) .
\end{aligned}
$$

\footnotetext{
${ }^{1}$ Repeated indices are summed over unless explicitly specified.
} 
The free-energy density is given by

$$
f\left(L_{0}, \boldsymbol{\xi}, \theta_{0}\right)=-\frac{1}{L_{0} V} \ln Z\left(L_{0}, \boldsymbol{\xi}, \theta_{0}\right),
$$

where $V=L_{1} L_{2} L_{3}$ is the spatial volume of the box. In the thermodynamic limit, which is always assumed in this section, the invariance of the dynamics under the $\mathrm{SO}(4)$ group implies [11]

$$
f\left(L_{0}, \boldsymbol{\xi}, \theta_{0}\right)=f\left(L_{0} \sqrt{1+\boldsymbol{\xi}^{2}}, \mathbf{0}, \theta_{0}\right),
$$

where the parameter $\theta_{0}$ remains the same on the two sides of the equation since the conserved quark number charge is a relativistic invariant. The effect of the shift is therefore to lower the physical temperature and the chemical potential of the system of the same factor, i.e. from $T=L_{0}^{-1}$ to $T=\left(L_{0} \sqrt{1+\xi^{2}}\right)^{-1}$ and from $\mu_{\mathcal{I}}$ to $\mu_{\mathcal{I}} / \sqrt{1+\xi^{2}}$ respectively, with respect to the system with the same values of $L_{0}$ and $\mu_{\mathcal{I}}$ but no shift. The entropy density reads

$$
\frac{s}{T^{3}}=-L_{0}^{4}\left(1+\boldsymbol{\xi}^{2}\right)^{2}\left\{\frac{\left(1+\boldsymbol{\xi}^{2}\right)}{\xi_{k}}\left\langle T_{0 k}\right\rangle_{\boldsymbol{\xi}, \theta_{0}}+i \mu_{\mathcal{I}}\left\langle V_{0}\right\rangle_{\boldsymbol{\xi}, \theta_{0}}\right\}
$$

where $V_{0}=\bar{\psi} \gamma_{0} \psi$. This formula generalizes the one in ref. [11] to the case of a non-zero chemical potential. The expectation values of the space-time components of the energymomentum tensor dictate the dependence on $\boldsymbol{\xi}$ of the free-energy density to be [9-11]

$$
\left\langle T_{0 k}\right\rangle_{\boldsymbol{\xi}, \theta_{0}}=-\frac{\partial}{\partial \xi_{k}} f\left(L_{0}, \boldsymbol{\xi}, \theta_{0}\right),
$$

while its $\theta_{0}$-dependence is determined by the average value of the temporal component of the vector current so to satisfy

$$
\left\langle V_{0}\right\rangle_{\boldsymbol{\xi}, \theta_{0}}=-i L_{0} \frac{\partial}{\partial \theta_{0}} f\left(L_{0}, \boldsymbol{\xi}, \theta_{0}\right) .
$$

As a result, the significant dependence of $\left\langle T_{0 k}\right\rangle_{\boldsymbol{\xi}, \theta_{0}}$ on $\theta_{0}$ can be written as

$$
\left\langle T_{0 k}\right\rangle_{\boldsymbol{\xi}, \theta_{0}^{A}}-\left\langle T_{0 k}\right\rangle_{\boldsymbol{\xi}, \theta_{0}^{B}}=\frac{i}{L_{0}} \int_{\theta_{0}^{A}}^{\theta_{0}^{B}} d \theta_{0} \frac{\partial}{\partial \xi_{k}}\left\langle V_{0}\right\rangle_{\boldsymbol{\xi}, \theta_{0}}
$$

a relation which turns out to be useful on the lattice.

The expectation values of the traceless diagonal components of the energy-momentum tensor are related to those of the space-time components via the Lorentz transformation discussed in sections 2 and 4 of ref. [11], e.g. (no summation over repeated indices)

$$
\begin{aligned}
\left\langle T_{0 k}\right\rangle_{\boldsymbol{\xi}, \theta_{0}} & =\frac{\xi_{k}}{1-\xi_{k}^{2}}\left\{\left\langle T_{00}\right\rangle_{\boldsymbol{\xi}, \theta_{0}}-\left\langle T_{k k}\right\rangle_{\boldsymbol{\xi}, \theta_{0}}\right\}, \\
\left\langle T_{0 k}\right\rangle_{\boldsymbol{\xi}, \theta_{0}} & =\xi_{k}\left\{\left\langle T_{00}\right\rangle_{\boldsymbol{\xi}, \theta_{0}}-\left\langle T_{j j}\right\rangle_{\boldsymbol{\xi}, \theta_{0}}\right\} \quad\left(j \neq k, \xi_{j}=0\right) .
\end{aligned}
$$

Such relations can be imposed on the lattice to fix their relative normalization. Finally for the trace it holds [13]

$$
\frac{\partial}{\partial \xi_{k}}\left\langle T_{\mu \mu}\right\rangle_{\boldsymbol{\xi}, \theta_{0}}=\frac{1}{\left(1+\boldsymbol{\xi}^{2}\right)^{2}} \frac{\partial}{\partial \xi_{k}}\left[\frac{\left(1+\boldsymbol{\xi}^{2}\right)^{3}}{\xi_{k}}\left\langle T_{0 k}\right\rangle_{\boldsymbol{\xi}, \theta_{0}}\right]
$$


By combining eq. (2.13) with eqs. (2.14)-(2.16), the $\theta_{0}$-dependence of the expectation values of the diagonal components of $T_{\mu \nu}$ can be related to the one of the vector current as well.

\subsection{Finiteness of $T_{\mu \nu}$ and trace anomaly}

In dimensional regularization the energy-momentum tensor (2.1) is decomposed as

$$
T_{\mu \nu}=\tau_{\mu \nu}+\frac{1}{4} \delta_{\mu \nu} \bar{\tau}
$$

with

$$
\tau_{\mu \nu}=\tau_{\mu \nu}^{G}+\tau_{\mu \nu}^{F}
$$

where

$$
\begin{aligned}
\tau_{\mu \nu}^{G} & =\frac{1}{g_{0}^{2}}\left\{F_{\mu \alpha}^{a} F_{\nu \alpha}^{a}-\frac{1}{D} \delta_{\mu \nu} F_{\alpha \beta}^{a} F_{\alpha \beta}^{a}\right\} \\
\tau_{\mu \nu}^{F} & =\frac{1}{4}\left\{\bar{\psi} \gamma_{\mu} \overleftrightarrow{D}_{\nu} \psi+\bar{\psi} \gamma_{\nu} \overleftrightarrow{D}_{\mu} \psi\right\}-\frac{1}{D} \delta_{\mu \nu} \bar{\psi}\left\{\frac{1}{2} \gamma_{\alpha} \overleftrightarrow{D}_{\alpha}\right\} \psi
\end{aligned}
$$

The singlet operator is

$$
\bar{\tau}=\bar{\tau}^{G}+\bar{\tau}^{F}
$$

where

$$
\bar{\tau}^{G}=\frac{2 \epsilon}{D g_{0}^{2}} F_{\alpha \beta}^{a} F_{\alpha \beta}^{a},
$$

and up to terms that vanish by the equation of motion of the fermion fields

$$
\bar{\tau}^{F}=-\frac{4}{D} \bar{\psi} M_{0} \psi
$$

with $D=4-2 \epsilon$.

\subsubsection{The non-singlet}

The dimension-four gauge-invariant fields $\tau_{\mu \nu}^{G}$ and $\tau_{\mu \nu}^{F}$ are parity and charge conjugation invariant, and transform as a two-index traceless symmetric irreducible representation of the $\mathrm{SO}(D)$ group. No other gauge-invariant field with the same quantum numbers and dimension $\leq 4$ can be constructed. Since the derivative of the free-energy density with respect to the shift is finite once the bare parameters of the theory have been renormalized [9-11], i.e. eq. (2.11), we may choose the renormalization pattern to be

$$
\left(\begin{array}{c}
\tau_{\mu \nu}^{G} \\
\tau_{\mu \nu}^{F}
\end{array}\right)=\mathcal{Z}_{\tau}\left(\begin{array}{c}
\tau_{\mu \nu}^{G, R} \\
\tau_{\mu \nu}^{F, R}
\end{array}\right), \quad \mathcal{Z}_{\tau}=\left(\begin{array}{cc}
1+z_{g} & -z_{f} \\
-z_{g} & 1+z_{f}
\end{array}\right),
$$

where the superscript $R$ labels renormalized quantities in the desired scheme. The anomalous-dimension matrix takes the form

$$
\gamma_{\tau}(g)=-\frac{1}{\mathcal{Z}_{\tau}} \mu \frac{d}{d \mu} \mathcal{Z}_{\tau}=\left(\begin{array}{cc}
a & c \\
-a & -c
\end{array}\right)=g^{2} \sum_{k=0}^{\infty}\left(\gamma_{\tau}\right)_{k} g^{2 k}
$$


where at leading order in $g^{2}$ the coefficient matrix is

$$
\left(\gamma_{\tau}\right)_{0}=\left(\begin{array}{cc}
a_{0} & c_{0} \\
-a_{0} & -c_{0}
\end{array}\right)=R_{0}^{-1}\left(\begin{array}{cc}
\gamma_{0} & 0 \\
0 & 0
\end{array}\right) R_{0}, \quad R_{0}=\left(\begin{array}{cc}
\frac{2 a_{0}}{\gamma_{0}} & \frac{2 c_{0}}{\gamma_{0}} \\
1 & 1
\end{array}\right),
$$

with $[6,24]$

$$
a_{0}=-\frac{1}{(4 \pi)^{2}} \frac{4 N_{f}}{3}, \quad c_{0}=\frac{1}{(4 \pi)^{2}} \frac{8}{3} \frac{N_{c}^{2}-1}{N_{c}}, \quad \gamma_{0}=\left(a_{0}-c_{0}\right) .
$$

It is then straightforward to verify that

$$
\gamma_{\tau}=R_{0}^{-1}\left(\begin{array}{ll}
\gamma & \alpha \\
0 & 0
\end{array}\right) R_{0}
$$

where

$$
\alpha=2 \frac{c a_{0}-a c_{0}}{\gamma_{0}}=g^{4} \sum_{k=0}^{\infty} \alpha_{k+1} g^{2 k}, \quad \gamma=a-c=g^{2} \sum_{k=0}^{\infty} \gamma_{k} g^{2 k}
$$

By following refs. [25-27], we can define the RGI gluonic and fermionic components of the energy-momentum tensor as

$$
\left(\begin{array}{c}
\tau_{\mu \nu}^{G, \mathrm{RGI}} \\
\tau_{\mu \nu}^{F, \mathrm{RGI}}
\end{array}\right)=\Theta_{\tau}(g)\left(\begin{array}{c}
\tau_{\mu \nu}^{G, R} \\
\tau_{\mu \nu}^{F, R}
\end{array}\right)
$$

where $\Theta_{\tau}(g)$ satisfies the differential equation

$$
\beta(g) \frac{\partial}{\partial g} \Theta_{\tau}(g)+\Theta_{\tau}(g) \gamma_{\tau}(g)=0
$$

with the initial condition ${ }^{2}$

$$
\lim _{g \rightarrow 0}\left\{\Theta_{\tau}(g)\left(2 b_{0} g^{2}\right)^{-\frac{\left(\gamma_{\tau}\right)_{0}}{2 b_{0}}}-\frac{\alpha_{1} g^{2}}{2\left(2 b_{0}+\gamma_{0}\right)}\left(2 b_{0} g^{2}\right)^{\frac{\gamma_{0}}{2 b_{0}}}\left(\begin{array}{cc}
1 & 1 \\
-1 & -1
\end{array}\right)\right\}=\mathbb{1},
$$

and with $b_{0}$ being the first coefficient of the $\beta$-function defined in eq. (B.16). The solution has the same structure as $\mathcal{Z}_{\tau}$, and it can be written as

$$
\Theta_{\tau}(g)=R_{0}^{-1}\left(\begin{array}{cc}
\theta_{1} & \theta_{2} \\
0 & 1
\end{array}\right) R_{0}
$$

where

$$
\begin{aligned}
& \theta_{1}(g)=\left(2 b_{0} g^{2}\right)^{\frac{\gamma_{0}}{2 b_{0}}} \exp \left\{-\int_{0}^{g}\left[\frac{\gamma(\bar{g})}{\beta(\bar{g})}+\frac{\gamma_{0}}{b_{0} \bar{g}}\right] d \bar{g}\right\} \\
& \theta_{2}(g)=\frac{\alpha_{1} g^{2}}{2 b_{0}+\gamma_{0}}\left(2 b_{0} g^{2}\right)^{\frac{\gamma_{0}}{2 b_{0}}}-\int_{0}^{g}\left[\frac{\alpha(\bar{g})}{\beta(\bar{g})} \theta_{1}(\bar{g})+\frac{\alpha_{1} \bar{g}}{b_{0}}\left(2 b_{0} \bar{g}^{2}\right)^{\frac{\gamma_{0}}{2 b_{0}}}\right] d \bar{g}
\end{aligned}
$$

\footnotetext{
${ }^{2}$ For $\gamma_{0} / b_{0}>-2$, e.g. $N_{c}=3$ and $N_{f} \leq 5$, the subtraction proportional to $\alpha_{1}$ in eq. (2.32) is harmless. If $\gamma_{0} / b_{0} \leq-4$, e.g. $N_{c}=3$ and $N_{f}>9$, further subtractions are required.
} 
and the $\beta$-function $\beta(g)$ is defined in eq. (B.15). From these equations it follows that

$$
\begin{aligned}
\tau_{\mu \nu} & =\tau_{\mu \nu}^{G, \mathrm{RGI}}+\tau_{\mu \nu}^{F, \mathrm{RGI}}, \\
t_{\mu \nu} & =\tau_{\mu \nu}^{G, \mathrm{RGI}}-\tau_{\mu \nu}^{F, \mathrm{RGI}}
\end{aligned}
$$

are two independent RGI fields. Once defined, they allow for an unambiguous scale- and scheme-independent separation of the energy-momentum tensor in two parts, and therefore of its form factors among physical states, which tend to the free gluonic and fermionic contributions when $g \rightarrow 0$. At finite temperature, for instance, this allows for an unambiguous split of the entropy density in two parts which tend, in the infinite temperature limit, to the Stefan-Boltzmann values for gluons and fermions respectively.

\subsubsection{The singlet}

The gluonic and fermionic components of $\bar{\tau}$ are singlets under $\mathrm{SO}(D)$, and therefore they mix with the identity. A natural prescription for subtracting this contribution leads to the renormalization pattern

$$
\left(\begin{array}{c}
\frac{1}{g_{0}^{2}} F_{\alpha \beta}^{a} F_{\alpha \beta}^{a}-\left\langle\frac{1}{g_{0}^{2}} F_{\alpha \beta}^{a} F_{\alpha \beta}^{a}\right\rangle_{0} \\
\bar{\psi} M_{0} \psi-\left\langle\bar{\psi} M_{0} \psi\right\rangle_{0}
\end{array}\right)=\mathcal{Z}_{\bar{\tau}}\left(\begin{array}{c}
\left\{\frac{1}{g_{0}^{2}} F_{\alpha \beta}^{a} F_{\alpha \beta}^{a}\right\}^{R} \\
\left\{\bar{\psi} M_{0} \psi\right\}^{R}
\end{array}\right),
$$

where $\langle\ldots\rangle_{0}$ indicates the expectation value for $L_{0} \rightarrow \infty$ (zero temperature), and

$$
\mathcal{Z}_{\bar{\tau}}=\left(\begin{array}{cc}
1+\bar{z}_{g} & \bar{z}_{f} \\
0 & 1
\end{array}\right) \text {. }
$$

Also for the singlet, we can define the RGI gluonic and fermionic operators as

$$
\left(\begin{array}{c}
\left\{\frac{1}{g_{0}^{2}} F_{\alpha \beta}^{a} F_{\alpha \beta}^{a}\right\}^{\mathrm{RGI}} \\
\left\{\bar{\psi} M_{0} \psi\right\}^{\mathrm{RGI}}
\end{array}\right)=\Theta_{\bar{\tau}(g)}\left(\begin{array}{c}
\left\{\frac{1}{g_{0}^{2}} F_{\alpha \beta}^{a} F_{\alpha \beta}^{a}\right\}^{R} \\
\left\{\bar{\psi} M_{0} \psi\right\}^{R}
\end{array}\right),
$$

where the $2 \times 2$ matrix $\Theta_{\bar{\tau}}(g)$ satisfies the differential equation

$$
\beta(g) \frac{\partial}{\partial g} \Theta_{\bar{\tau}}(g)+\Theta_{\bar{\tau}}(g) \gamma_{\bar{\tau}}(g)=0
$$

with the initial condition

$$
\lim _{g \rightarrow 0}\left\{\Theta_{\bar{\tau}}(g)\left(2 b_{0} g^{2}\right)^{\frac{-\left(\gamma_{\bar{\tau}}\right)_{0}}{2 b_{0}}}\right\}=\mathbb{1} .
$$

The anomalous-dimension matrix is given by

$$
\gamma_{\bar{\tau}}(g)=-\frac{1}{\mathcal{Z}_{\bar{\tau}}} \mu \frac{d}{d \mu} \mathcal{Z}_{\bar{\tau}}=\left(\begin{array}{cc}
\bar{\gamma} & \bar{\alpha} \\
0 & 0
\end{array}\right)=g^{2} \sum_{k=0}^{\infty}\left(\gamma_{\bar{\tau}}\right)_{k} g^{2 k},
$$

where at leading order in $g^{2}$, see below, the coefficient matrix is

$$
\left(\gamma_{\bar{\tau}}\right)_{0}=\left(\begin{array}{cc}
2 b_{0} & -4 d_{0} \\
0 & 0
\end{array}\right)
$$


The solution of eqs. (2.41) and (2.42), analogous to the one in eqs. (2.33)-(2.35), reads

$$
\Theta_{\bar{\tau}}(g)=\left(\begin{array}{cc}
\bar{\theta}_{1} & \bar{\theta}_{2} \\
0 & 1
\end{array}\right)
$$

where

$$
\begin{aligned}
& \bar{\theta}_{1}(g)=\left(2 b_{0} g^{2}\right) \exp \left\{-\int_{0}^{g}\left[\frac{\bar{\gamma}(\bar{g})}{\beta(\bar{g})}+\frac{2}{\bar{g}}\right] d \bar{g}\right\}, \\
& \bar{\theta}_{2}(g)=\frac{2 d_{0}}{b_{0}}-\int_{0}^{g} \frac{\bar{\alpha}(\bar{g})}{\beta(\bar{g})} \bar{\theta}_{1}(\bar{g}) d \bar{g} .
\end{aligned}
$$

In dimensional regularization, by deriving eq. (2.11) with respect to the renormalized coupling at fixed renormalized quark mass $M$, see eqs. (2.8), (B.11) and (B.12), we obtain

$$
2 g \frac{\partial}{\partial g}\left\langle T_{0 k}\right\rangle_{\boldsymbol{\xi}, \theta_{0}}=-\frac{\epsilon g}{\beta(\epsilon, g)} \frac{\partial}{\partial \xi_{k}}\left\langle\frac{1}{g_{0}^{2}} F_{\alpha \beta}^{a} F_{\alpha \beta}^{a}\right\rangle_{\boldsymbol{\xi}, \theta_{0}}+\frac{2 g \gamma_{m}}{\beta(\epsilon, g)} \frac{\partial}{\partial \xi_{k}}\left\langle\bar{\psi} M_{0} \psi\right\rangle_{\boldsymbol{\xi}, \theta_{0}}
$$

where $\gamma_{m}$ is the anomalous dimension of the quark mass defined in eq. (B.17). Since the l.h.s. of (2.48) is finite, the r.h.s. defines the renormalized gluonic operator in eq. (2.38) with the choice

$$
\bar{z}_{g}=-\frac{\beta(g)}{\epsilon g}, \quad \bar{z}_{f}=\frac{2 \gamma_{m}}{\epsilon} .
$$

Analogously by deriving with respect to the renormalized quark masses, it follows that we can take $\left(\mathcal{Z}_{\bar{\tau}}\right)_{22}=1$. These values of the renormalization constants correspond exactly to the MS prescription, and therefore the superscript $R$ in eq. (2.38) labels renormalized fields defined in the MS scheme in this case. The anomalous dimension matrix reads

$$
\gamma_{\bar{\tau}}(g)=-\beta(\epsilon, g) \frac{1}{\mathcal{Z}_{\bar{\tau}}} \frac{\partial}{\partial g} \mathcal{Z}_{\bar{\tau}}=g \frac{\partial}{\partial g}\left(\begin{array}{cc}
-\frac{\beta(g)}{g} 2 \gamma_{m} \\
0 & 0
\end{array}\right) .
$$

By inserting these findings in eq. (2.21), and taking the limit $\epsilon \rightarrow 0$, one obtains the result $[28-30]$

$$
\bar{\tau}-\langle\bar{\tau}\rangle_{0}=-\frac{\beta(g)}{2 g}\left\{\frac{1}{g_{0}^{2}} F_{\alpha \beta}^{a} F_{\alpha \beta}^{a}\right\}^{R}-\left(1-\gamma_{m}\right)\left\{\bar{\psi} M_{0} \psi\right\}^{R},
$$

which is valid to all orders in perturbation theory. By noticing that the solution of eqs. (2.41) and (2.42) is

$$
\Theta_{\bar{\tau}}(g)=\left(\begin{array}{cc}
-\frac{2 \beta(g)}{g} & {\left[\frac{2 d_{0}}{b_{0}}+4 \gamma_{m}\right]} \\
0 & 1
\end{array}\right)
$$

we can finally rewrite eq. (2.51) for the trace anomaly in terms of RGI operators as

$$
\bar{\tau}-\langle\bar{\tau}\rangle_{0}=\frac{1}{4}\left\{\frac{1}{g_{0}^{2}} F_{\alpha \beta}^{a} F_{\alpha \beta}^{a}\right\}^{\mathrm{RGI}}-\left(1+\frac{d_{0}}{2 b_{0}}\right)\left\{\bar{\psi} M_{0} \psi\right\}^{\mathrm{RGI}} .
$$


Analogously to the non-singlet case, we can write

$$
\bar{\tau}-\langle\bar{\tau}\rangle_{0}=\bar{\tau}^{G, \mathrm{RGI}}+\bar{\tau}^{F, \mathrm{RGI}},
$$

where

$$
\begin{aligned}
\bar{\tau}^{G, \mathrm{RGI}} & =\frac{1}{4}\left\{\frac{1}{g_{0}^{2}} F_{\alpha \beta}^{a} F_{\alpha \beta}^{a}\right\}^{\mathrm{RGI}}-\frac{d_{0}}{2 b_{0}}\left\{\bar{\psi} M_{0} \psi\right\}^{\mathrm{RGI}} \\
\bar{\tau}^{F, \mathrm{RGI}} & =-\left\{\bar{\psi} M_{0} \psi\right\}^{\mathrm{RGI}} .
\end{aligned}
$$

Considerations analogous to those made for the non-singlet components of the energymomentum tensor apply also in this case.

\section{$2.3 \quad$ Finite volume}

In a finite volume the theory needs to be further specified by the boundary conditions imposed on the fields in the spatial directions. For the gauge field we supplement eq. (2.6) by standard periodic boundary conditions in the spatial directions,

$$
A_{\mu}\left(x_{0}, \boldsymbol{x}+\hat{k} L_{k}\right)=A_{\mu}\left(x_{0}, \boldsymbol{x}\right),
$$

where $\hat{k}$ is the unit vector in the direction $k$. Note that this implies $-L_{k} / 2 \leq L_{0} \xi_{k}<L_{k} / 2$. For quark and anti-quark fields the boundary conditions in eqs. (2.7) are supplemented by periodic boundary conditions in the spatial directions up to a non-trivial twist phase $\theta_{k}$ [31]

$$
\begin{aligned}
\psi\left(x_{0}, \boldsymbol{x}+\hat{k} L_{k}\right) & =e^{i \theta_{k}} \psi\left(x_{0}, \boldsymbol{x}\right), \\
\bar{\psi}\left(x_{0}, \boldsymbol{x}+\hat{k} L_{k}\right) & =e^{-i \theta_{k}} \bar{\psi}\left(x_{0}, \boldsymbol{x}\right) .
\end{aligned}
$$

The finite length of the spatial directions and the angles $\theta_{k}$ softly break the $\mathrm{SO}(4)$ group, and $\theta_{k}$ breaks charge conjugation as well. As a consequence eqs. (2.14)-(2.16) are modified in a finite volume.

For instance, eq. (2.14) needs to be replaced by eq. (4.25) of ref. [11]. In the latter there is an extra term proportional to the expectation value of $T_{0 k}$ computed in the reference frame where the velocity in direction $k$ is null while the other velocity components are unchanged. For this extra term to be null, the space-time geometry must satisfy the condition [11]

$$
\frac{L_{k} \xi_{k}}{L_{0}\left(1+\xi_{k}^{2}\right)}=q \in \mathbb{Z}
$$

together with

$$
b_{0}=\boldsymbol{\xi} \cdot \boldsymbol{b}-\xi_{k} b_{k} \quad \text { or } \quad b_{0}=\boldsymbol{\xi} \cdot \boldsymbol{b}+\frac{b_{k}}{\xi_{k}},
$$

where $b_{\mu}=\theta_{\mu} / L_{\mu}$. Similarly eq. (2.15) picks up two extra terms: one proportional to the expectation value of $T_{0 k}$ as before, and a second one proportional to the expectation value of $\left(T_{k k}-T_{j j}\right)$. For both these terms to be null in the reference frame where the velocity 
in direction $k$ is null while the other components are unchanged, the condition (2.59) and $L_{k} / \sqrt{1+\xi_{k}^{2}}=L_{j}$ have to be met together with

$$
\left\{\begin{array} { l } 
{ b _ { 0 } = \boldsymbol { \xi } \cdot \boldsymbol { b } - \xi _ { k } b _ { k } } \\
{ b _ { k } = \frac { L _ { j } } { L _ { k } } b _ { j } }
\end{array} \quad \text { or } \quad \left\{\begin{array}{l}
b_{0}=\boldsymbol{\xi} \cdot \boldsymbol{b}+\frac{b_{k}}{\xi_{k}} \\
b_{j}=0
\end{array} .\right.\right.
$$

In the simplified case in which the only non-null component of the shift is $\xi_{k}$, the conditions in eqs. (2.59)-(2.61) can be summarized as

$$
\frac{L_{k} \xi_{k}}{L_{0}\left(1+\xi_{k}^{2}\right)}=q, \quad\left\{\begin{array} { l } 
{ \theta _ { 0 } = 0 } \\
{ \theta _ { k } = \theta _ { j } }
\end{array} \quad \text { or } \quad \left\{\begin{array}{l}
\theta_{k}=q \theta_{0} \\
\theta_{j}=0
\end{array} .\right.\right.
$$

It must be said, however, that in the presence of a mass gap the extra terms acquired by eqs. (2.14)-(2.16) vanish exponentially with the length of the spatial directions, and therefore they are expected to be negligible in large enough volumes.

\section{The energy-momentum tensor on the lattice}

A non-perturbative definition of the theory presented in section 2 is achieved by introducing a four-dimensional Euclidean hypercubic lattice of spacing $a$ which acts as an ultraviolet regulator. The gauge potential $A_{\mu}$ is replaced as usual by the $\mathrm{SU}\left(N_{c}\right)$-valued gauge fields $U_{\mu}$ residing on the links of the lattice, while the quark and anti-quark fields $\psi, \bar{\psi}$ are defined on the sites. ${ }^{3}$ Although the non-perturbative renormalization strategy presented in this paper is general, for definiteness we consider the Wilson formulation for gluons and quarks, see appendix $\mathrm{C}$ for the details.

A discretization of the energy-momentum tensor is obtained by replacing the fields and the derivatives appearing in the continuum expressions (2.1)-(2.3) with their lattice counterparts. In particular we define [1-4]

$$
T_{\mu \nu}=T_{\mu \nu}^{G}+T_{\mu \nu}^{F},
$$

with the gluonic component given by

$$
T_{\mu \nu}^{G}=\frac{1}{g_{0}^{2}}\left\{F_{\mu \alpha}^{a} F_{\nu \alpha}^{a}-\frac{1}{4} \delta_{\mu \nu} F_{\alpha \beta}^{a} F_{\alpha \beta}^{a}\right\}
$$

where

$$
F_{\mu \nu}^{a}=2 \operatorname{Tr}\left\{\widehat{F}_{\mu \nu} T^{a}\right\}, \quad F_{\mu \nu}=F_{\mu \nu}^{a} T^{a},
$$

and with $\widehat{F}_{\mu \nu}$ being the clover discretization of the field strength tensor in eq. (C.9) which, at variance with $F_{\mu \nu}$, is not traceless. For the fermionic part we take

$$
\begin{aligned}
T_{\mu \nu}^{F}= & \frac{1}{8}\left\{\bar{\psi} \gamma_{\mu}\left[\overleftrightarrow{\nabla}_{\nu}^{*}+\overleftrightarrow{\nabla}_{\nu}\right] \psi+\bar{\psi} \gamma_{\nu}\left[\overleftrightarrow{\nabla}_{\mu}^{*}+\overleftrightarrow{\nabla}_{\mu}\right] \psi\right\} \\
& -\frac{1}{4} \delta_{\mu \nu} \bar{\psi}\left\{\frac{1}{4} \gamma_{\alpha}\left(\overleftrightarrow{\nabla}_{\alpha}^{*}+\overleftrightarrow{\nabla}_{\alpha}\right)+M_{0}\right\} \psi,
\end{aligned}
$$

\footnotetext{
${ }^{3}$ We use the same notation for lattice and continuum quantities, since any ambiguity is resolved from the context. As usual, the continuum limit value of a renormalized lattice quantity, identified with the superscript $R$, is the one to be identified with its continuum counterpart.
} 
where $\overleftrightarrow{\nabla}_{\mu}$ and $\overleftrightarrow{\nabla}_{\mu}^{*}$ are defined in eq. (C.12). In the naive continuum limit $a \rightarrow 0$, these expressions tend to the continuum ones in eqs. (2.2) and (2.3). As any other discretization of $T_{\mu \nu}$, however, they need to be properly renormalized to guarantee that their correlation functions satisfy the continuum Ward identities, up to discretization errors which vanish in the continuum limit.

The target energy-momentum tensor in the continuum is a gauge-invariant operator of dimension 4, which is a combination of a traceless two-index symmetric and a singlet irreducible representations of $\mathrm{SO}(4)$ even under parity and charge conjugation. Since on the lattice the $\mathrm{SO}(4)$ symmetry reduces to the hypercubic group $\mathrm{SW}_{4}$, the traceless twoindex symmetric representation splits into a sextet (non-diagonal components) and a triplet (diagonal traceless components). At finite lattice spacing, the energy-momentum tensor is thus a combination of gauge-invariant operators of dimension $d \leq 4$ which, under the hypercubic group, transform as one of those two representations and the singlet [1]. In QCD there are seven such operators plus the identity. We can take as a basis (no summation over the repeated indices $\mu$ and $\nu$ in eqs. (3.6) and (3.9))

$$
\begin{aligned}
T_{\mu \nu}^{G,\{6\}} & =\left(1-\delta_{\mu \nu}\right) \frac{1}{g_{0}^{2}}\left\{F_{\mu \alpha}^{a} F_{\nu \alpha}^{a}\right\}, \\
T_{\mu \nu}^{G,\{3\}} & =\frac{1}{g_{0}^{2}}\left\{F_{\mu \alpha}^{a} F_{\mu \alpha}^{a}-F_{\nu \alpha}^{a} F_{\nu \alpha}^{a}\right\}, \\
T_{\mu \nu}^{G,\{1\}} & =\delta_{\mu \nu} \frac{1}{4 g_{0}^{2}} F_{\alpha \beta}^{a} F_{\alpha \beta}^{a},
\end{aligned}
$$

for the purely gluonic fields and

$$
\begin{aligned}
T_{\mu \nu}^{F,\{6\}} & =\left(1-\delta_{\mu \nu}\right) \frac{1}{8}\left\{\bar{\psi} \gamma_{\mu}\left[\overleftrightarrow{\nabla}_{\nu}^{*}+\overleftrightarrow{\nabla}_{\nu}\right] \psi+\bar{\psi} \gamma_{\nu}\left[\overleftrightarrow{\nabla}_{\mu}^{*}+\overleftrightarrow{\nabla}_{\mu}\right] \psi\right\}, \\
T_{\mu \nu}^{F,\{3\}} & =\frac{1}{4}\left\{\bar{\psi} \gamma_{\mu}\left[\overleftrightarrow{\nabla}_{\mu}^{*}+\overleftrightarrow{\nabla}_{\mu}\right] \psi-\bar{\psi} \gamma_{\nu}\left[\overleftrightarrow{\nabla}_{\nu}^{*}+\overleftrightarrow{\nabla}_{\nu}\right] \psi\right\}, \\
T_{\mu \nu}^{F,\{1 a\}} & =\delta_{\mu \nu} \frac{1}{16} \bar{\psi}\left\{\gamma_{\alpha}\left(\overleftrightarrow{\nabla}_{\alpha}^{*}+\overleftrightarrow{\nabla}_{\alpha}\right)\right\} \psi, \\
T_{\mu \nu}^{F,\{1 b\}} & =\delta_{\mu \nu} \bar{\psi} \psi,
\end{aligned}
$$

for the fermionic ones. ${ }^{4}$ Since the hypercubic group is an exact symmetry of the lattice theory, a given field in eqs. (3.5)-(3.11) can mix under renormalization only with those in the same irreducible representation.

In this paper we focus on the definition of the sextet and triplet whose renormalization pattern is given by

$$
T_{\mu \nu}^{R,\{i\}}=Z_{G}^{\{i\}}\left(g_{0}^{2}\right) T_{\mu \nu}^{G,\{i\}}+Z_{F}^{\{i\}}\left(g_{0}^{2}\right) T_{\mu \nu}^{F,\{i\}}, \quad i=6,3,
$$

where the renormalization constants $Z_{G}^{\{i\}}$ and $Z_{F}^{\{i\}}$ are finite and depend on $g_{0}^{2}$ only since in the continuum the nonet component of the energy-momentum tensor has vanishing anomalous dimension. In a regularization which breaks chiral symmetry explicitly, the

\footnotetext{
${ }^{4}$ Notice that, at variance with the continuum, the operator $T_{\mu \nu}^{F,\{1 b\}}$ is not a chiral singlet, i.e. multiplied by the quark mass, since chiral symmetry is explicitly broken for Wilson fermions.
} 
renormalization pattern of the singlet component requires a rather different and more involved analysis which fills a different publication by itself. Departure from scale invariance can, however, be extracted directly from the Callan-Symanzik renormalization group equations or, for instance, from the r.h.s. of eq. (2.16). Finally we note that, as anticipated in section 2.2.1, the determination of the renormalized fields

$$
t_{\mu \nu}^{R,\{i\}}(\mu)=z_{G}^{\{i\}}\left(g_{0}^{2}, a \mu\right) T_{\mu \nu}^{G,\{i\}}-z_{F}^{\{i\}}\left(g_{0}^{2}, a \mu\right) T_{\mu \nu}^{F,\{i\}}, \quad i=6,3,
$$

and in particular of their RGI counterparts, gives access to the RGI gluonic and fermionic components of the energy-momentum tensor. While the renormalization of $T_{\mu \nu}^{\{i\}}$ is fixed by WIs, that of (3.13) can be obtained, for instance, by imposing suitable conditions on its expectation values in the presence of shifted and twisted boundary conditions. A detail investigation of this problem is in progress.

\subsection{Non-perturbative renormalization conditions}

The renormalization constants $Z_{G}^{\{i\}}$ and $Z_{F}^{\{i\}}$ can be determined non-perturbatively by enforcing on the lattice the relations (2.11) and (2.14) up to discretization effects which vanish in the continuum limit. To this aim, shifted boundary conditions for the links are

$$
U_{\mu}\left(x_{0}+L_{0}, \boldsymbol{x}\right)=U_{\mu}\left(x_{0}, \boldsymbol{x}-L_{0} \boldsymbol{\xi}\right), \quad U_{\mu}\left(x_{0}, \boldsymbol{x}+\hat{k} L_{k}\right)=U_{\mu}\left(x_{0}, \boldsymbol{x}\right),
$$

while eqs. (2.7) and (2.58) fix the boundary conditions for quark and anti-quark fields on the lattice too, where the components of $\boldsymbol{\xi}$ are discretized in integer units of $a / L_{0}$. As very commonly adopted in lattice QCD, we opt for a mass-independent renormalization scheme. The renormalized coupling $g_{R}$ and the renormalized quark mass $m_{R}$ for each given flavour are thus related to the bare parameters as

$$
g_{R}^{2}=Z_{g}\left(g_{0}^{2}, a \mu\right) g_{0}^{2}, \quad m_{R}=Z_{m}\left(g_{0}^{2}, a \mu\right) m_{q},
$$

where $\mu$ is a renormalization scale, $m_{q}=m_{0}-m_{c}\left(g_{0}^{2}\right)$ is the subtracted mass, and $m_{c}\left(g_{0}^{2}\right)$ is the critical mass.

The two renormalization constants of the sextet are fixed by requiring that, for two different set of values $\theta^{A}$ and $\theta^{B}$, where $\theta=\left(\theta_{0}, \boldsymbol{\theta}\right)$, it holds

$$
\left\langle T_{0 k}^{R,\{6\}}\right\rangle_{\boldsymbol{\xi}, \theta}=-\frac{\Delta f\left(L_{0}, \boldsymbol{\xi}, \theta\right)}{\Delta \xi_{k}},
$$

where

$$
\frac{\Delta f\left(L_{0}, \boldsymbol{\xi}, \theta\right)}{\Delta \xi_{k}}=\frac{L_{0}}{2 a}\left[f\left(L_{0}, \boldsymbol{\xi}+\frac{a}{L_{0}} \hat{k}, \theta\right)-f\left(L_{0}, \boldsymbol{\xi}-\frac{a}{L_{0}} \hat{k}, \theta\right)\right]
$$

is a symmetric discrete approximation of the derivative of the free energy with respect to the $k$-th component of the shift. From a practical point of view, it is useful to combine eq. (3.16) for one set of values of the angles, e.g. $\theta=\theta^{A}$, with the lattice realization of the identity (2.13)

$$
\left\langle T_{0 k}^{R,\{6\}}\right\rangle_{\boldsymbol{\xi}, \theta^{A}}-\left\langle T_{0 k}^{R,\{6\}}\right\rangle_{\boldsymbol{\xi}, \theta^{B}}=\frac{i}{L_{0}} \int_{\theta_{0}^{A}}^{\theta_{0}^{B}} d \theta_{0} \frac{\Delta\left\langle V_{0}^{R}\right\rangle_{\boldsymbol{\xi}, \theta}}{\Delta \xi_{k}}, \quad \boldsymbol{\theta}^{A}=\boldsymbol{\theta}^{B},
$$


since the r.h.s. can be computed very efficiently by Monte Carlo simulations. Moreover, if one takes the temporal component of the flavour-singlet conserved lattice vector current

$$
V_{\mu}^{c}(x)=\frac{1}{2}\left\{\bar{\psi}(x+a \hat{\mu}) U_{\mu}^{\dagger}(x)\left(\gamma_{\mu}+1\right) \psi(x)+\bar{\psi}(x) U_{\mu}(x)\left(\gamma_{\mu}-1\right) \psi(x+a \hat{\mu})\right\},
$$

then

$$
V_{\mu}^{R}(x)=V_{\mu}^{c}(x),
$$

and the expectation value of the renormalized current on the r.h.s. of eq. (3.18) corresponds to the bare one. ${ }^{5}$

Once the sextet renormalization constants have been fixed, those of the triplet can be determined by enforcing on the lattice the analogous of eqs. (2.14) or (2.15)

$$
\begin{aligned}
\left\langle T_{0 k}^{R,\{6\}}\right\rangle_{\boldsymbol{\xi}, \theta} & =\frac{\xi_{k}}{1-\xi_{k}^{2}}\left\langle T_{0 k}^{R,\{3\}}\right\rangle_{\boldsymbol{\xi}, \theta} \\
\left\langle T_{0 k}^{R,\{6\}}\right\rangle_{\boldsymbol{\xi}, \theta} & =\xi_{k}\left\langle T_{0 j}^{R,\{3\}}\right\rangle_{\boldsymbol{\xi}, \theta}, \quad\left(j \neq k, \xi_{j}=0\right),
\end{aligned}
$$

for two different set of values $\theta=\theta^{A}, \theta^{B}$ of the twist angles. In a finite volume these relations are satisfied only up to exponentially small finite-size effects, unless lattice sizes and twist phases according to the constraints given in eqs. (2.59)-(2.61) are considered. The above renormalization conditions are imposed at zero quark masses, i.e. all bare masses are set to the critical value $m_{0}=m_{c r}\left(g_{0}\right)$. In practice this is possible thanks to the presence of a spectral gap in the lattice Dirac operator at finite temperature.

Finally, it is important to emphasize that the above renormalization conditions are valid non-perturbatively, and are designed to be accessible to numerical Monte Carlo computations. The eq. (3.16) can be studied numerically with a strategy analogous to the one already successfully implemented for the Yang-Mills theory [13], while eqs. (3.18) and (3.21) require standard numerical techniques. In this sense these conditions provide a practical strategy to define non-perturbatively the energy-momentum tensor in QCD.

\section{$4 \mathrm{O}(a)$-improvement}

The Symanzik improvement programme has the purpose of accelerating the approach to the continuum limit of field correlators. It is achieved by adding suitable counterterms to the lattice action and to the fields multiplied by numerical coefficients which are properly adjusted so to cancel discretization errors order by order in the lattice spacing [32, 33]. In the following we discuss how to implement this programme to $\mathrm{O}(a)$-improve on-shell matrix elements of the sextet and triplet components of the energy-momentum tensor. For the clarity of the presentation, we discuss separately the massless, mass-degenerate, and mass non-degenerate cases.

\footnotetext{
${ }^{5}$ It is interesting to notice that the renormalization constant and the improvement coefficients of the flavour-singlet lattice vector currents can be determined by comparing $\left\langle V_{0}^{c}\right\rangle_{\boldsymbol{\xi}, \theta}$ with the analogous one for the local current and similarly for higher-point correlation functions.
} 


\subsection{Massless quarks}

The first step consists in identifying complete bases of dimension-5 gauge-invariant fields which, in the Symanzik effective continuum theory, are parity and charge conjugation invariant and transform as sextets and triplets under the $\mathrm{SO}(4)$ hypercubic subgroup. When all quarks are massless, i.e. $m_{0}=m_{c}\left(g_{0}\right)$, there are no operators made of the gauge field only. Complete bases of $\mathrm{O}(a)$-counterterms are built by projecting the fields

$$
\bar{\psi} \sigma_{\mu \rho} F_{\nu \rho} \psi, \quad \partial_{\rho}\left\{\bar{\psi} \sigma_{\mu \rho} \stackrel{\leftrightarrow}{D}_{\nu} \psi\right\}, \quad \partial_{\mu} \partial_{\nu}\{\bar{\psi} \psi\}
$$

on their sextet and triplet components. Terms proportional to the second and third fields in (4.1) do not contribute to matrix elements between initial and final states with the same four-momentum. Since we restrict our analysis to those cases, we can discard them. The improved sextet and triplet fields are then obtained by replacing

$$
T_{\mu \nu}^{F,\{i\}} \longrightarrow T_{\mu \nu}^{F,\{i\}}+a \delta T_{\mu \nu}^{F,\{i\}}, \quad i=6,3
$$

in eqs. (3.8) and (3.9) respectively, with (no summation over $\mu, \nu$ in (4.4))

$$
\begin{aligned}
& \delta T_{\mu \nu}^{F,\{6\}}=c_{F}^{\{6\}}\left(g_{0}^{2}\right) \frac{1}{8}\left(1-\delta_{\mu \nu}\right) \bar{\psi}\left[\sigma_{\mu \rho} F_{\nu \rho}+\sigma_{\nu \rho} F_{\mu \rho}\right] \psi, \\
& \delta T_{\mu \nu}^{F,\{3\}}=c_{F}^{\{3\}}\left(g_{0}^{2}\right) \frac{1}{4} \bar{\psi}\left[\sigma_{\mu \rho} F_{\mu \rho}-\sigma_{\nu \rho} F_{\nu \rho}\right] \psi .
\end{aligned}
$$

By performing a classical expansion of $T_{\mu \nu}^{F,\{i\}}$ in the lattice spacing [34], it turns out that the tree-level values of the coefficients $c_{F}^{\{i\}}$ are null as well as those multiplying the other counteterms in eq. (4.1).

\subsection{Mass-degenerate quarks}

When all quarks have the same non-vanishing mass, the renormalization of the coupling and of the mass can be obtained from eqs. (3.15) by replacing

$$
g_{0}^{2} \longrightarrow \widetilde{g}_{0}^{2}=g_{0}^{2}\left(1+b_{g}\left(g_{0}^{2}\right) a m_{q}\right), \quad m_{q} \longrightarrow \widetilde{m}_{q}=m_{q}\left(1+b_{m}\left(g_{0}^{2}\right) a m_{q}\right),
$$

where $m_{q}$ is the subtracted bare mass common to all flavours. The two improvement coefficients $b_{g}$ and $b_{m}$ must be chosen so to have a mass-independent renormalization scheme where the renormalized coupling and the mass are free from $\mathrm{O}(a)$-effects [31]. Defined this way, both coefficients do not depend on the renormalization conditions chosen to set $Z_{g}$ and $Z_{m}$. The perturbative expansion of $b_{g}$ starts at $\mathrm{O}\left(g_{0}^{2}\right)$ since it arises from sea-quark loop contributions to a gluonic quantity [35].

To improve the sextet and triplet parts of $T_{\mu \nu}$, two more $\mathrm{O}(a)$-counterterms, made of the original gluon and fermion components multiplied by the quark mass, have to be taken into account. As a result, the $\mathrm{O}(a)$-improved fields $\mathrm{read}^{6}$

$$
\begin{aligned}
T_{\mu \nu}^{R,\{i\}}= & Z_{G}^{\{i\}}\left(\widetilde{g}_{0}^{2}\right)\left(1+b_{G}^{\{i\}}\left(g_{0}^{2}\right) a m_{q}\right) T_{\mu \nu}^{G,\{i\}} \\
& +Z_{F}^{\{i\}}\left(\widetilde{g}_{0}^{2}\right)\left(1+b_{F}^{\{i\}}\left(g_{0}^{2}\right) a m_{q}\right)\left\{T_{\mu \nu}^{F,\{i\}}+a \delta T_{\mu \nu}^{F,\{i\}}\right\}, \quad i=6,3 .
\end{aligned}
$$

\footnotetext{
${ }^{6}$ We use the same symbol for the unimproved and the improved energy-momentum tensor field since any ambiguity is resolved from the context.
} 
Notice that the renormalization constants appearing in eq. (4.6) must be evaluated at the value $\widetilde{g}_{0}^{2}$. On the other hand, it is consistent to evaluate $c_{F}^{\{i\}}$ and the two extra coefficients $b_{G}^{\{i\}}$ and $b_{F}^{\{i\}}$ either at $\widetilde{g}_{0}^{2}$ or $g_{0}^{2}$. The term proportional to $m_{q} \delta T_{\mu \nu}^{F,\{i\}}$ in eq. (4.6) can be neglected since is $\mathrm{O}\left(a^{2}\right)$. The coefficients $b_{G}^{\{i\}}$ are null at tree-level because they arise from sea-quark loop contributions.

\subsection{Mass non-degenerate quarks}

When all quark masses are different, the pattern of improvement is further complicated. $\mathrm{An} \mathrm{O}(a)$-improved renormalized coupling can be defined from eqs. (3.15) by replacing [36]

$$
g_{0}^{2} \longrightarrow \widetilde{g}_{0}^{2}=g_{0}^{2}\left(1+\frac{b_{g}\left(g_{0}^{2}\right)}{N_{f}} \operatorname{tr}\left\{a M_{q}\right\}\right), \quad M_{q}=M_{0}-m_{c}\left(g_{0}^{2}\right) \mathbb{1}
$$

while the expressions for the renormalized improved quark masses are quite involved and, since they are not needed in the following, we refer the interested reader directly to eq. (26) of ref. [36]. The $\mathrm{O}(a)$-improved fields turn out to be

$$
\begin{aligned}
T_{\mu \nu}^{R,\{i\}}=Z_{G}^{\{i\}}\left(\widetilde{g}_{0}^{2}\right)(1 & \left.+\frac{b_{G}^{\{i\}}\left(g_{0}^{2}\right)}{N_{f}} \operatorname{tr}\left\{a M_{q}\right\}\right) T_{\mu \nu}^{G,\{i\}}+Z_{F}^{\{i\}}\left(\widetilde{g}_{0}^{2}\right)\left[\hat{b}_{F}^{\{i\}}\left(g_{0}^{2}\right) \sum_{f, g=1}^{N_{f}} a M_{q}^{f g} T_{\mu \nu ; g f}^{F,\{i\}}\right. \\
& \left.+\left(1+\frac{\bar{b}_{F}^{\{i\}}\left(g_{0}^{2}\right)}{N_{f}} \operatorname{tr}\left\{a M_{q}\right\}\right)\left\{T_{\mu \nu}^{F,\{i\}}+a \delta T_{\mu \nu}^{F,\{i\}}\right\}\right], \quad i=6,3, \quad
\end{aligned}
$$

where $T_{\mu \nu ; f g}^{F,\{i\}}$ indicates the flavour non-singlet analogous of $T_{\mu \nu}^{F,\{i\}}$, e.g. $\left(f, g=1, \ldots, N_{f}\right)$

$$
T_{\mu \nu ; f g}^{F,\{6\}}=\left(1-\delta_{\mu \nu}\right) \frac{1}{8}\left\{\bar{\psi}^{f} \gamma_{\mu}\left[\overleftrightarrow{\nabla}_{\nu}^{*}+\overleftrightarrow{\nabla}_{\nu}\right] \psi^{g}+\bar{\psi}^{f} \gamma_{\nu}\left[\overleftrightarrow{\nabla}_{\mu}^{*}+\overleftrightarrow{\nabla}_{\mu}\right] \psi^{g}\right\}
$$

and analogously for $T_{\mu \nu ; f g}^{F,\{3\}}$. In the mass-degenerate limit $M_{q}=m_{q} \mathbb{1}$, the expressions of the previous subsection are recovered, up to $\mathrm{O}\left(a^{2} m\right)$ effects, provided one sets $b_{F}^{\{i\}}=\hat{b}_{F}^{\{i\}}+\bar{b}_{F}^{\{i\}}$. In perturbation theory the coefficients $\bar{b}_{F}^{\{i\}}$ are null up to 1-loop order since they originate from sea-quark loop contributions to fermionic quantities.

\subsection{Improvement conditions}

As we have seen in the previous subsections, improving the energy-momentum tensor may require the tuning of several parameters. A decoupling of the equations that fix them, however, occurs naturally within our strategy. Indeed, in the massless limit chiral symmetry is expected to be either exact or effectively restored when the temperature $T$ is much larger than the typical scale of the strong interactions, a few hundred $\mathrm{MeV}$ or so. As a consequence, the expectation values of the chiral non-singlet counterterms in eq. (4.1) either vanish or become quickly negligible at high temperature. The origin of this result can be traced back to the more general fact that the thermal theory with massless quarks enjoys de-facto automatic $O(a)$-improvement at high temperature, see appendix $\mathrm{D}$ for a detailed discussion in the presence of a generic number of flavours. 
For the particular case we are concerned here, namely the expectation values of the energy-momentum tensor, stronger results can be proven. ${ }^{7}$ In the Symanzik effective continuum theory with two or more massless flavours, it holds

$$
\left\langle\delta T_{\mu \nu}^{F,\{i\}}\right\rangle_{\boldsymbol{\xi}, \theta_{0}}=-\frac{N_{f}}{N_{f}^{2}-1} \int_{\partial R} d \sigma_{k}\left\langle A_{k}^{a}(x) \delta T_{\mu \nu}^{a, F,\{i\}}(0)\right\rangle_{\boldsymbol{\xi}, \theta_{0}},
$$

where $\partial R$ is the union of the top and bottom lids of an hyper-cylinder $R$ containing the origin, $A_{k}^{a}=\bar{\psi} \gamma_{k} \gamma_{5} T^{a} \psi(k=1,2,3)$ with $T^{a}\left(a=1, \ldots, N_{f}^{2}-1\right)$ being the generators of the group $\mathrm{SU}\left(N_{f}\right)$, and $\delta T_{\mu \nu}^{a, F,\{i\}}$ is defined as $\delta T_{\mu \nu}^{F,\{i\}}$ but with the replacement $\psi \rightarrow \gamma_{5} T^{a} \psi$. At large temperature, where the theory has a mass gap, the integrand in eq. (4.10) decreases exponentially with the distance $|x|$. Its integral is therefore null exactly since the lids can be sent to infinity, and the conclusions of appendix D are recovered. At smaller temperature, where the theory develops Goldstone bosons [37], the integrand decreases power-like in $|x|$. At large distances, the leading behaviour is dictated by the single Goldstone-boson contribution so that

$$
\left\langle A_{\rho}^{a}(x) \delta T_{\mu \nu}^{a, F,\{i\}}(0)\right\rangle_{\boldsymbol{\xi}, \theta_{0}} \propto \partial_{\rho}\left(\partial_{\mu} \partial_{\nu}-\frac{1}{4} \delta_{\mu \nu} \square\right) \Delta(x)+\ldots
$$

where $\Delta(x)$ is the free propagator of a massless boson while the dots indicate sub-leading corrections. At distances $|x|$ much larger than the inverse temperature, $\Delta(x) \propto 1 /|x|$, the correlator (4.11) decreases as $|x|^{-4}$, and the surface integral in (4.10) is again null.

The final important outcome of this analysis is that, if the lattice action is $\mathrm{O}(a)$-improved and quarks are massless, the expectation values $\left\langle T_{\mu \nu}^{F,\{i\}}\right\rangle_{\boldsymbol{\xi}, \theta_{0}}$ are $\mathrm{O}(a)$ improved at any temperature as well as the renormalization constants fixed by imposing eqs. (3.16), (3.21) or (3.22).

The improvement coefficients in eq. (4.8) can be determined non-perturbatively by imposing the very same equations $(3.16),(3.21)$ or (3.22) to be valid up to $\mathrm{O}(a)$ terms for several values of the quark masses, and by remembering that the free-energy density is already improved once the Sheikholeslami-Wohlert term has been included in the action. A detailed implementation of this strategy to 1-loop order in perturbation theory is discussed in section 5.2.3.

\section{Perturbative analysis}

In order to verify analytically the validity of the strategy proposed in this paper, we have computed the free-energy density and the expectation values of the energy-momentum tensor components to 1-loop order in lattice perturbation theory in the presence of shifted and twisted boundary conditions in the infinite spatial volume limit. The calculation has been carried out by regularizing gluons with the Wilson plaquette action and quarks with the $O(a)$-improved Wilson operator, see appendices $\mathrm{C}$ and $\mathrm{E}$ for the definitions of the actions, free propagators, and lattice vertices. This computation serves also to determine, for the first time, the renormalization constants of the sextet and triplet components of $T_{\mu \nu}$

\footnotetext{
${ }^{7}$ We thank Martin Lüscher for suggesting to us this line of argumentation.
} 
in the $\mathrm{O}(a)$-improved theory to 1-loop order in perturbation theory, as well as their $O(a)$ improvement coefficients. As a byproduct we have confirmed the 1-loop expressions of the renormalization constants in the unimproved theory which were determined in refs. [4-8]. Finally the combination of the results in this section with eqs. (3.16), (3.21) or (3.22) leads to perturbatively improved versions of these non-perturbative renormalization conditions. As a consequence, the numerical non-perturbative determinations of the renormalization constants are free from discretization effects up to order $g_{0}^{2}$.

\subsection{Free-energy density}

The 1-loop expansion of the bare free-energy density defined in eq. (2.8) is

$$
f\left(L_{0}, \boldsymbol{\xi}, \theta\right)=f^{(0)}+g_{0}^{2} f^{(1)},
$$

where

$$
f^{(0)}=\left(N_{c}^{2}-1\right) f^{G(0)}+N_{c} N_{f} f^{F(0)},
$$

and

$$
f^{(1)}=\left(N_{c}^{2}-1\right)\left[N_{c} f^{G\left(1, N_{c}\right)}+\frac{1}{N_{c}} f^{G\left(1, \frac{1}{N_{c}}\right)}+N_{f} f^{F\left(1, N_{f}\right)}\right]
$$

are the tree-level and 1-loop contributions respectively. ${ }^{8}$ The functions $f^{G(0)}$ and $f^{F(0)}$ are the tree-level gluonic and fermionic contributions, $f^{G\left(1, N_{c}\right)}$ and $f^{G\left(1, \frac{1}{N_{c}}\right)}$ are the 1-loop gluonic parts, and $f^{F\left(1, N_{f}\right)}$ collects the 1-loop fermionic contributions. All these functions are reported in appendix F. In the perturbative computations presented in this paper we assume always to have $N_{f}$ quarks with equal masses and twist angles. The formulas for the generic case, however, can be easily obtained by summing the contributions of each individual flavour rather than multiplying the single-fermion contribution by $N_{f}$, e.g for the free-energy density the terms $N_{f} f^{F(0)}$ and $N_{f} f^{F\left(1, N_{f}\right)}$ must be replaced by the sums over the flavours of the $f^{F(0)}$ and $f^{F\left(1, N_{f}\right)}$ functions computed for the mass and the twist angles of each single flavour respectively.

Once the bare free-energy density has been calculated, the 1-loop renormalized expression is obtained by re-writing the bare parameters $g_{0}$ and the common bare quark mass $m_{0}$ through the renormalized counterparts defined in eq. (3.15). By properly combining eq. (2.8), the renormalized 1-loop expression at finite lattice spacing and its continuum limit, a 1-loop perturbative improved definition of the free-energy density can also be obtained.

\subsection{Energy-momentum tensor}

The bare expectation values of the sextet and triplet components of $T_{\mu \nu}$ are

$$
\left\langle T_{\mu \nu}^{\{i\}}\right\rangle_{\boldsymbol{\xi}, \theta}=\mathcal{T}_{\mu \nu}^{\{i\}(0)}+g_{0}^{2} \mathcal{T}_{\mu \nu}^{\{i\}(1)}, \quad i=6,3,
$$

where the tree-level values are

$$
\mathcal{T}_{\mu \nu}^{\{i\}(0)}=\left(N_{c}^{2}-1\right) \mathcal{T}_{\mu \nu}^{G\{i\}(0)}+N_{c} N_{f} \mathcal{T}_{\mu \nu}^{F\{i\}(0)},
$$

\footnotetext{
${ }^{8}$ Throughout this section the arguments of the various tree-level and 1-loop contributions such as $\boldsymbol{\xi}, m_{0}$, etc. are omitted for better readability.
} 
while the 1-loop contributions are

$$
\mathcal{T}_{\mu \nu}^{\{i\}(1)}=\left(N_{c}^{2}-1\right)\left[N_{c} \mathcal{T}_{\mu \nu}^{G\{i\}\left(1, N_{c}\right)}+\frac{1}{N_{c}} \mathcal{T}_{\mu \nu}^{G\{i\}\left(1, \frac{1}{N_{c}}\right)}+N_{f} \mathcal{T}_{\mu \nu}^{G\{i\}\left(1, N_{f}\right)}+N_{f} \mathcal{T}_{\mu \nu}^{F\{i\}\left(1, N_{f}\right)}\right]
$$

All functions on the r.h.s. of eqs. (5.5)-(5.6) are given in appendices $\mathrm{H}$ and I.

Once the bare parameters of the theory have been renormalized, the definition of the sextet and the triplet components of $T_{\mu \nu}$ require the calculation of the renormalization constants defined in eq. (3.12). At one loop they can be written as

$$
Z_{G}^{\{i\}}=Z_{G}^{\{i\}(0)}+g_{0}^{2} Z_{G}^{\{i\}(1)}, \quad Z_{F}^{\{i\}}=Z_{F}^{\{i\}(0)}+g_{0}^{2} Z_{F}^{\{i\}(1)}, \quad i=6,3,
$$

where we can define

$$
\begin{aligned}
& Z_{G}^{\{i\}(1)}=N_{c} Z_{G}^{\{i\}\left(1, N_{c}\right)}+\frac{1}{N_{c}} Z_{G}^{\{i\}\left(1, \frac{1}{N_{c}}\right)}+N_{f} Z_{G}^{\{i\}\left(1, N_{f}\right)}, \\
& Z_{F}^{\{i\}(1)}=\frac{\left(N_{c}^{2}-1\right)}{N_{c}} Z_{F}^{\{i\}\left(1, N_{c}\right)}
\end{aligned}
$$

To impose the renormalization conditions in eqs. (3.16), (3.21) or (3.22) in the massless limit $m_{R}=0$, we remind that at 1-loop the critical mass is

$$
m_{c}=m_{c}^{(0)}+m_{c}^{(1)} g_{0}^{2}
$$

where $m_{c}^{(0)}=0$ and [38]

$$
\begin{aligned}
m_{c}^{(1)}= & \frac{\left(N_{c}^{2}-1\right)}{N_{c}} m_{c}^{\left(1, N_{c}\right)} \\
a m_{c}^{\left(1, N_{c}\right)}= & -0.16285705871085(1)+c_{\mathrm{sw}} 0.04348303388205(10) \\
& +c_{\mathrm{sw}}^{2} 0.01809576878142(1) .
\end{aligned}
$$

At the order we work, $c_{\mathrm{sw}}=0$ or 1 for the unimproved and improved theory respectively. The chiral limit in the expressions (5.1)-(5.6) is then reached by requiring that $m_{0}=$ $m_{c}^{(1)} g_{0}^{2}$. In practice, to 1-loop order, that corresponds to Taylor expand in the bare quark mass the tree-level expressions of the observables, and then to fix $m_{0}=m_{c}^{(0)}=0$ and to replace

$$
\begin{aligned}
f^{F\left(1, N_{f}\right)} & \longrightarrow f^{F\left(1, N_{f}\right)}+\frac{\partial f^{F(0)}}{\partial m_{0}} m_{c}^{\left(1, N_{c}\right)} \\
\mathcal{T}_{\mu \nu}^{F\{i\}\left(1, N_{f}\right)} & \longrightarrow \mathcal{T}_{\mu \nu}^{F\{i\}\left(1, N_{f}\right)}+\frac{\partial \mathcal{T}_{\mu \nu}^{F\{i\}(0)}}{\partial m_{0}} m_{c}^{\left(1, N_{c}\right)}, \quad i=6,3,
\end{aligned}
$$

where the derivatives on the r.h.s. of these equations can be found in eqs. (F.2), (H.17) and (I.6) respectively. 


\subsubsection{Renormalization constants of the sextet}

By imposing eq. (3.16) for two different values ${ }^{9}$ of $\theta_{0}=\theta_{0}^{A}, \theta_{0}^{B}$, the tree-level values can be defined as

$$
Z_{G}^{\{6\}(0)}=-\frac{1}{\mathcal{T}_{0 k}^{G\{6\}(0)}} \frac{\Delta f^{G(0)}}{\Delta \xi_{k}} \quad \text { and } \quad Z_{F}^{\{6\}(0)}=-\frac{1}{\mathcal{T}_{0 k}^{F\{6\}(0)}} \frac{\Delta f^{F(0)}}{\Delta \xi_{k}}
$$

where the discrete derivative $\Delta$ with respect to the shift is defined as in eq. (3.17). As expected, in the limit $L_{0} / a \rightarrow \infty$ it holds $Z_{G}^{\{6\}(0)}=Z_{F}^{\{6\}(0)}=1$. The pure gluonic 1-loop contributions are

$$
Z_{G}^{\{6\}\left(1, N_{c}\right)}=-\frac{1}{\mathcal{T}_{0 k}^{G\{6\}(0)}}\left\{Z_{G}^{\{6\}(0)} \mathcal{T}_{0 k}^{G\{6\}\left(1, N_{c}\right)}+\frac{\Delta f^{G\left(1, N_{c}\right)}}{\Delta \xi_{k}}\right\},
$$

and $Z_{G}^{\{6\}\left(1, \frac{1}{N_{c}}\right)}$ which has the very same expression as $Z_{G}^{\{6\}\left(1, N_{c}\right)}$ once $\left(1, N_{c}\right) \rightarrow\left(1, \frac{1}{N_{c}}\right)$.

The last two terms, $Z_{G}^{\{6\}\left(1, N_{f}\right)}$ and $Z_{F}^{\{6\}\left(1, N_{c}\right)}$, depend on the interaction between quarks and gluons. If we define the combination

$$
d^{\{6\}}=-\left\{Z_{G}^{\{6\}(0)} \mathcal{T}_{0 k}^{G\{6\}\left(1, N_{f}\right)}+Z_{F}^{\{6\}(0)} \mathcal{T}_{0 k}^{F\{6\}\left(1, N_{f}\right)}+\frac{\Delta f^{F\left(1, N_{f}\right)}}{\Delta \xi_{k}}\right\}
$$

we obtain

$$
Z_{G}^{\{6\}\left(1, N_{f}\right)}=\frac{1}{\mathcal{T}_{0 k}^{G\{6\}(0)}}\left\{d^{\{6\}}-Z_{F}^{\{6\}\left(1, N_{c}\right)} \mathcal{T}_{0 k}^{F\{6\}(0)}\right\},
$$

where the renormalization constant of the fermion component is

$$
Z_{F}^{\{6\}\left(1, N_{c}\right)}=\frac{d^{\{6\}}\left(\theta_{0}^{A}\right)-d^{\{6\}}\left(\theta_{0}^{B}\right)}{\mathcal{T}_{0 k}^{F\{6\}(0)}\left(\theta_{0}^{A}\right)-\mathcal{T}_{0 k}^{F\{6\}(0)}\left(\theta_{0}^{B}\right)},
$$

and in the last equation we have explicitly indicated the dependence on $\theta_{0}$ since is the only one where two different values are needed.

The values of the various terms which define $Z_{G}^{\{6\}}$ and $Z_{F}^{\{6\}}$ have been computed numerically on lattices with temporal extension $L_{0} / a$ ranging from 4 to 32 in steps of 2 and spatial size $L_{1}=L_{2}=L_{3}=R L_{0}$ with $R=6,8,10$ and 12 . The calculations have been carried out for two values of the shift, $\boldsymbol{\xi}=(1,0,0)$ and $(1 / 2,1 / 2,0)$, and for three values of the fermionic phase in the temporal direction, $\theta_{0}=0, \pi / 16$ and $\pi / 4$. At fixed value of the shift, data for the three values of $\theta_{0}$ have been analyzed by considering two independent differences. This large amount of data allowed us to extrapolate the results to infinite spatial volume and to $a / L_{0} \rightarrow 0$ limit with confidence. The latter extrapolation has been performed by fitting the results in powers of $\left(a / L_{0}\right)^{2}$ supplemented by terms multiplied by $\ln \left(a / L_{0}\right)$ for the 1-loop coefficients. The required lattice sums have been computed in coordinate space [39] after having used the Fast Fourier Transform algorithm for computing the gluon and quark propagators. A hierarchical procedure for sums has been implemented

\footnotetext{
${ }^{9}$ In perturbation theory we always set $\boldsymbol{\theta}=\mathbf{0}$ since we work in the infinite spatial volume limit.
} 


\begin{tabular}{|c|cc|cc|}
\hline & \multicolumn{2}{|c|}{ Sextet $(i=6)$} & \multicolumn{2}{c|}{ Triplet $(i=3)$} \\
& This work & Ref. [4-8] & This work & Ref. [4-8] \\
\hline$Z_{G}^{\{i\}(0)}$ & $1.000000(5)$ & 1 & $1.000000(5)$ & 1 \\
$Z_{G}^{\{i\}\left(1, N_{c}\right)}$ & $0.10414(3)$ & 0.10413887 & $0.09773(3)$ & 0.09772334 \\
$Z_{G}^{\{i\}\left(1, \frac{1}{N_{c}}\right)}$ & $-0.125000(1)$ & $-1 / 8$ & $-0.157495(1)$ & -0.15749516 \\
$Z_{G, c_{\mathrm{sw}}=0}^{\{i\}\left(1, N_{f}\right)}$ & $0.010827(1)$ & 0.01082699 & $0.00601(2)$ & 0.006010835 \\
$Z_{G, c_{\mathrm{sw}}=1}^{\left\{i, N_{f}\right)}$ & $0.0301785(25)$ & - & $0.02466(2)$ & - \\
\hline$Z_{F}^{\{i\}(0)}$ & $1.000000(5)$ & 1 & $1.000000(5)$ & 1 \\
$Z_{F, c_{\mathrm{sw}}=0}^{\left\{i\left(1, N_{c}\right)\right.}$ & $-0.01474(1)$ & -0.01473 & $-0.03167(3)$ & -0.03169 \\
$Z_{F, c_{\mathrm{sw}}=1}^{\left\{i\left(N_{c}\right)\right.}$ & $0.005282(4)$ & - & $-0.0109(1)$ & - \\
\hline
\end{tabular}

Table 1. Numerical values of the coefficients of the perturbative expressions of $Z_{G}^{\{i\}}$ and $Z_{F}^{\{i\}}$ computed in this work for $c_{\mathrm{sw}}=0$ and 1 corresponding to the unimproved and improved theory respectively. Results from refs. [4-8] are also shown for comparison.

in order to preserve a high numerical accuracy. For some of them, however, we needed to run in quadruple precision due to large cancellations taking place at large volumes.

The final results for the various contributions to $Z_{G}^{\{6\}}$ and $Z_{F}^{\{6\}}$ in the limit $a / L_{0} \rightarrow 0$ are listed in table 1 together with the analogous values present in the literature [4-8]. Our error bars have been estimated by changing the fit range in $\left(a / L_{0}\right)$, by considering the spread over the two differences in $\theta_{0}$, and by analyzing the dependence on $R$. For all values that can be compared with refs. [4-8], the agreement is excellent.

\subsubsection{Renormalization constants of the triplet}

By combining eqs. (3.16) and (3.21) or (3.22), the renormalization constants of the triplet can be determined analogously to the previous subsection. The eqs. (5.15)-(5.19) hold provided the representation index changes, $6 \rightarrow 3$, and the various components of $\mathcal{T}_{0 k}$ are multiplied by $\xi_{k} /\left(1-\xi_{k}^{2}\right)$ or $\xi_{k}$ when eq. (3.21) or (3.22) are used respectively. A different option is, however, possible. Once the renormalization constants of the sextet have been computed, the ones of the triplet can be determined directly imposing eq. (3.21) or (3.22) for two different values of $\theta_{0}=\theta_{0}^{A}, \theta_{0}^{B}$. In this first case the tree-level, values are

$$
Z_{G}^{\{3\}(0)}=Z_{G}^{\{6\}(0)} \frac{1-\xi_{k}^{2}}{\xi_{k}} \frac{\mathcal{T}_{0 k}^{G\{6\}(0)}}{\mathcal{T}_{0 k}^{G\{3\}(0)}}, \quad Z_{F}^{\{3\}(0)}=Z_{F}^{\{6\}(0)} \frac{1-\xi_{k}^{2}}{\xi_{k}} \frac{\mathcal{T}_{0 k}^{F\{6\}(0)}}{\mathcal{T}_{0 k}^{F\{3\}(0)}},
$$

with $Z_{G}^{\{3\}(0)}=Z_{F}^{\{3\}(0)}=1$ in the limit $a / L_{0} \rightarrow 0$. The pure gluonic contributions are $Z_{G}^{\{3\}\left(1, N_{c}\right)}=Z_{G}^{\{6\}\left(1, N_{c}\right)} \frac{Z_{G}^{\{3\}(0)}}{Z_{G}^{\{6\}(0)}}+\frac{1}{\mathcal{T}_{0 k}^{G\{3\}(0)}}\left\{\frac{1-\xi_{k}^{2}}{\xi_{k}} Z_{G}^{\{6\}(0)} \mathcal{T}_{0 k}^{G\{6\}\left(1, N_{c}\right)}-Z_{G}^{\{3\}(0)} \mathcal{T}_{0 k}^{G\{3\}\left(1, N_{c}\right)}\right\}$,

and $Z_{G}^{\{3\}\left(1, \frac{1}{N_{c}}\right)}$ which has the very same expression as $Z_{G}^{\{3\}\left(1, N_{c}\right)}$ once $\left(1, N_{c}\right) \rightarrow\left(1, \frac{1}{N_{c}}\right)$. The last two terms, $Z_{G}^{\{3\}\left(1, N_{f}\right)}$ and $Z_{F}^{\{3\}\left(1, N_{c}\right)}$, depend on the interaction between quarks 
and gluons. If we define the combination

$$
\begin{aligned}
d^{\{3\}}= & Z_{G}^{\{6\}(0)} \mathcal{T}_{0 k}^{G\{6\}\left(1, N_{f}\right)}+Z_{F}^{\{6\}(0)} \mathcal{T}_{0 k}^{F\{6\}\left(1, N_{f}\right)}+Z_{G}^{\{6\}\left(1, N_{f}\right)} \mathcal{T}_{0 k}^{G\{6\}(0)} \\
& +Z_{F}^{\{6\}\left(1, N_{c}\right)} \mathcal{T}_{0 k}^{F\{6\}(0)}-\frac{\xi_{k}}{1-\xi_{k}^{2}}\left\{Z_{G}^{\{3\}(0)} \mathcal{T}_{0 k}^{G\{3\}\left(1, N_{f}\right)}+Z_{F}^{\{3\}(0)} \mathcal{T}_{0 k}^{F\{3\}\left(1, N_{f}\right)}\right\}
\end{aligned}
$$

we obtain

$$
Z_{G}^{\{3\}\left(1, N_{f}\right)}=\frac{1}{\mathcal{T}_{0 k}^{G\{3\}(0)}}\left\{\frac{1-\xi_{k}^{2}}{\xi_{k}} d^{\{3\}}-Z_{F}^{\{3\}\left(1, N_{c}\right)} \mathcal{T}_{0 k}^{F\{3\}(0)}\right\},
$$

where the renormalization constant of the fermion component is

$$
Z_{F}^{\{3\}\left(1, N_{c}\right)}=\frac{1-\xi_{k}^{2}}{\xi_{k}} \frac{d^{\{3\}}\left(\theta_{0}^{A}\right)-d^{\{3\}}\left(\theta_{0}^{B}\right)}{\mathcal{T}_{0 k}^{F\{3\}(0)}\left(\theta_{0}^{A}\right)-\mathcal{T}_{0 k}^{F\{3\}(0)}\left(\theta_{0}^{B}\right)},
$$

and in the last equation we have explicitly indicated the dependence on the set of twisted angles. It is important to notice that the values of the shift $\boldsymbol{\xi}$, and of the angles $\theta_{0}^{A}$ are $\theta_{0}^{B}$ used for fixing the sextet and the triplet renormalization constants can be in general different. If the renormalization condition (3.22) is used instead of (3.21), the eqs. (5.20)(5.24) remain valid but with the replacements $\xi_{k} /\left(1-\xi_{k}^{2}\right) \rightarrow \xi_{k}$ and $k \rightarrow j$ in the second subscript index of the triplet components.

The numerical value of the various contributions to $Z_{G}^{\{3\}}$ and $Z_{F}^{\{3\}}$ have been computed on lattices with temporal extension $L_{0} / a$ ranging from 4 to 32 in steps of 2 and spatial size $L_{1}=L_{2}=L_{3}=R L_{0}$ with $R=10$ and 15 . The calculations have been carried out for $\boldsymbol{\xi}=(2,0,0)$ so to satisfy the constraint in eq. (2.59). Three values of the fermionic phase, $\theta_{0}=0, \pi / 16$ and $\pi / 4$, have been considered and a non vanishing phase along the direction $\hat{1}$ has been chosen according to the third constraint in eq. (2.62). Analogously to the sextet case, data for three values of $\theta_{0}$ have been analyzed by considering two possible independent differences. The results show no relevant dependence from the spatial volume. The $a / L_{0} \rightarrow 0$ extrapolation has been performed again by fitting the results in powers of $\left(a / L_{0}\right)^{2}$ supplemented by terms multiplied by $\ln \left(a / L_{0}\right)$ for the 1-loop coefficients. As for the sextet, the lattice sums have been computed in coordinate space, and also in this case a hierarchical procedure was implemented.

The final results for the various contributions to $Z_{G}^{\{3\}}$ and $Z_{F}^{\{3\}}$ in the limit $a / L_{0} \rightarrow 0$ are listed in table 1 together with the analogous ones present in the literature [4-8]. The error bars have been estimated by changing the fit range in $\left(a / L_{0}\right)$, and by considering the spread over the two differences in $\theta_{0}$. Whenever a comparison with refs. [4-8] is possible, the agreement is excellent.

\subsubsection{Improvement coefficients}

We conclude the perturbative analysis of the strategy proposed in this paper by computing to 1-loop order the improvement coefficients of the sextet and triplet components of $T_{\mu \nu}$ introduced in eq. (4.8). The terms $\delta \mathcal{T}_{\mu \nu}^{F,\{i\}}$ do not contribute to this order, since the coefficients $c_{F}^{\{i\}}\left(g_{0}^{2}\right)$, as mentioned in section 4.1, are null at tree level as well as, for our 
choice of boundary conditions, the associated fields. The two $\bar{b}_{F}^{\{i\}}\left(g_{0}^{2}\right)$ vanish as well. The other coefficients in eq. (4.8), or equivalently eq. (4.6), can be written as

$$
b_{G}^{\{i\}}\left(g_{0}^{2}\right)=N_{f} b_{G}^{\{i\}\left(1, N_{f}\right)} g_{0}^{2}, \quad b_{F}^{\{i\}}\left(g_{0}^{2}\right)=1+\frac{N_{c}^{2}-1}{N_{c}} b_{F}^{\{i\}\left(1, N_{c}\right)} g_{0}^{2}, \quad i=6,3,
$$

where the tree-level values of the $b_{F}^{\{i\}}$ have been fixed so to remove the $\mathrm{O}(a)$ terms in the tree-level expressions of the fermion components of $T_{\mu \nu}$. They can be computed by imposing eqs. (3.16), (3.21) or (3.22) up to $\mathrm{O}(a)$ terms in the simpler case where all quarks have the same non-vanishing mass $m_{R} \neq 0$.

To this aim, by using eqs. (3.15) and (4.5), we remind that the $\mathrm{O}(a)$-improved quark mass is given by

$$
m_{R}=Z_{m}\left(g_{0}^{2}, a \mu\right)\left(1+b_{m}\left(g_{0}^{2}\right) a m_{q}\right) m_{q},
$$

where at 1-loop in perturbation theory [40]

$$
\begin{aligned}
Z_{m}\left(g_{0}^{2}, a \mu\right) & =1+\frac{N_{c}^{2}-1}{N_{c}} Z_{m}^{\left(1, N_{c}\right)} g_{0}^{2}, & Z_{m}^{\left(1, N_{c}\right)} & =-\frac{3}{(4 \pi)^{2}} \ln (a \mu), \\
b_{m}\left(g_{0}^{2}\right) & =-\frac{1}{2}+\frac{N_{c}^{2}-1}{N_{c}} b_{m}^{\left(1, N_{c}\right)} g_{0}^{2}, & b_{m}^{\left(1, N_{c}\right)} & =-0.036085(10) .
\end{aligned}
$$

Notice that the values of the improvement coefficients do not depend on the scale $\mu$. By remembering that $m_{q}=m_{0}-m_{c}\left(g_{0}^{2}\right)$, with $m_{c}\left(g_{0}^{2}\right)$ being the critical mass in eq. (5.10), one easily finds that the bare mass which solves eq. (5.26) at fixed renormalized quark mass is $m_{0}\left(g_{0}^{2}\right)=m_{0}^{(0)}+m_{0}^{(1)} g_{0}^{2}$ where [40]

$$
a m_{0}^{(0)}=1-\sqrt{1-2 a m_{R}}
$$

and

$$
\begin{aligned}
m_{0}^{(1)} & =\frac{N_{c}^{2}-1}{N_{c}} m_{0}^{\left(1, N_{c}\right)}, \\
a m_{0}^{\left(1, N_{c}\right)} & =a m_{c}^{\left(1, N_{c}\right)}-\frac{a m_{R} Z_{m}^{\left(1, N_{c}\right)}+2 b_{m}^{\left(1, N_{c}\right)}\left(1-a m_{R}-\sqrt{1-2 a m_{R}}\right)}{\sqrt{1-2 a m_{R}}} .
\end{aligned}
$$

The free-energy density and the expectation values of the energy-momentum tensor at bare mass $m_{0}\left(g_{0}\right)$ are obtained by evaluating the expressions $(5.1)-(5.6)$ at $m_{0}=m_{0}^{(0)}$ and by replacing

$$
\begin{aligned}
f^{F\left(1, N_{f}\right)} & \longrightarrow f^{F\left(1, N_{f}\right)}+\frac{\partial f^{F(0)}}{\partial m_{0}} m_{0}^{\left(1, N_{c}\right)} \\
\mathcal{T}_{\mu \nu}^{F\{i\}\left(1, N_{f}\right)} & \longrightarrow \mathcal{T}_{\mu \nu}^{F\{i\}\left(1, N_{f}\right)}+\frac{\partial \mathcal{T}_{\mu \nu}^{F\{i\}(0)}}{\partial m_{0}} m_{0}^{\left(1, N_{c}\right)}, \quad i=6,3,
\end{aligned}
$$

where the derivatives on the r.h.s. of these equations can be found in eqs. (F.2), (H.17) and (I.6) respectively. 


\begin{tabular}{|c|c|c|}
\hline & $\begin{array}{c}\text { Sextet } \\
i=6\end{array}$ & $\begin{array}{c}\text { Triplet } \\
i=3\end{array}$ \\
\hline$b_{G}^{\{i\}\left(1, N_{f}\right)}$ & $-0.012(1)$ & $-0.011(2)$ \\
$b_{F}^{\{i\}\left(1, N_{c}\right)}$ & $0.051(2)$ & $0.056(6)$ \\
\hline
\end{tabular}

Table 2. Numerical values of the coefficients $b_{G}^{\{i\}\left(1, N_{f}\right)}$ and $b_{F}^{\{i\}\left(1, N_{c}\right)}$ to 1-loop order.

The solutions of the eqs. (3.16), (3.21) or (3.22) at finite quark mass are obtained by replacing

$$
\begin{aligned}
Z_{F}^{\{i\}(0)} \longrightarrow & Z_{F}^{\{i\}(0)}\left(1+a m_{0}^{(0)}\right) \\
Z_{G}^{\{i\}\left(1, N_{f}\right)} \longrightarrow & Z_{G}^{\{i\}\left(1, N_{f}\right)}+Z_{G}^{\{i\}(0)} b_{G}^{\{i\}\left(1, N_{f}\right)} a m_{0}^{(0)} \\
Z_{F}^{\{i\}\left(1, N_{c}\right)} \longrightarrow & Z_{F}^{\{i\}\left(1, N_{c}\right)}\left(1+a m_{0}^{(0)}\right) \\
& +Z_{F}^{\{i\}(0)}\left(a m_{0}^{\left(1, N_{c}\right)}-a m_{c}^{\left(1, N_{c}\right)}+b_{F}^{\{i\}\left(1, N_{c}\right)} a m_{0}^{(0)}\right)
\end{aligned}
$$

in eqs. (5.15)-(5.24). The improvement coefficients are finally determined by inserting into the solutions the values of the renormalization constants obtained in the previous subsections, and then by solving for $b_{G}^{\{i\}\left(1, N_{f}\right)}$ and $b_{F}^{\{i\}\left(1, N_{c}\right)}$. In table 2 we list the numerical values of $b_{G}^{\{i\}\left(1, N_{f}\right)}$ and $b_{F}^{\{i\}\left(1, N_{c}\right)}$ that we have obtained in the limit $a / L_{0} \rightarrow 0$ by numerical computations and analyses analogous to those carried out in the previous two subsections.

\section{Conclusions}

Shifted boundary conditions in the presence of an imaginary chemical potential offer an extremely powerful tool to non-perturbatively renormalize composite operators on the lattice. In this work we have applied this framework to the case of the energy-momentum tensor. The strategy proposed here is the natural extension of the one already applied successfully to the SU(3) Yang-Mills theory [13]. The inclusion of quarks, however, complicates the problem because the gluonic and fermionic parts of the tensor mix together. Introducing a non-zero imaginary chemical potential gives the handle to solve that problem since, via a conserved charge, it couples differently to quark and gluons, in particular directly to quarks but only indirectly to gluons through their interaction with quarks. Ward identities can thus be written, both for the sextet and the triplet, which are different enough to resolve the mixing between the gluonic and the fermionic parts so that the computation becomes feasible non-perturbatively.

In view of that application and in order to check the whole construction, we have applied the method in lattice perturbation theory by computing the renormalization constants and the $\mathrm{O}(a)$-improvement coefficients of the sextet and triplet components of the energymomentum tensor to 1-loop order. The agreement with the results in the literature for the unimproved theory represents a very non-trivial test of the entire strategy proposed in this paper. A further confirmation of theory expectations is the $\boldsymbol{\xi}$ and $\theta$ independence of the 
renormalization constants once extrapolated to the $a / L_{0} \rightarrow 0$ limit. An important byproduct of these computations is the possibility of defining 1-loop perturbative improved estimators of the renormalization constants and of the expectation values of the energy-momentum tensor, so to reduce discretization effects in their non-perturbative determinations.

Once fixed so to satisfy the WIs, the renormalization constants are part of the definition of the energy-momentum field itself. Up to discretization errors, they do not depend on the particular correlator or kinematic conditions they were employed to fix them. They can be directly used to renormalize the energy-momentum tensor inserted at a physical distance from other fields in any correlator of QCD, e.g at zero or non-zero temperature with or without chemical potential.

\section{Acknowledgments}

We thank Martin Lüscher for many illuminating discussions, especially on topics in sections 2.2 and 4.4, and for comments on a preliminary version of this paper. The numerical integrals needed in lattice perturbation theory have been computed on the PC clusters Marconi at CINECA (CINECA-INFN and CINECA-Bicocca agreements) and Wilson at Milano-Bicocca. We thank these institutions for the computer resources and the technical support. We also acknowledge PRACE and ISCRA for awarding us access to MareNostrum at Barcelona Supercomputing Center (BSC), Spain (n. 2018194651) and to Marconi at CINECA (EoSQCD) respectively, where the non-perturbative computations are being performed. We acknowledge partial support by the INFN project "High performance data network".

\section{A Conventions and useful identities}

Here we summarize the conventions for the generators of the $\mathrm{SU}\left(N_{c}\right)$ group and for the Dirac matrices, $\gamma_{\mu}$, together with some standard identities that we have used in the 1loop perturbative computation. Let $T_{a}, a=1, \ldots,\left(N_{c}^{2}-1\right)$, be the hermitean traceless generators of the group $\mathrm{SU}\left(N_{c}\right)$ normalized as

$$
\operatorname{Tr}\left[T_{a} T_{b}\right]=\frac{1}{2} \delta_{a b}
$$

Their commutation and anti-commutation relations are ${ }^{10}$

$$
\left[T_{a}, T_{b}\right]=i f_{a b c} T_{c}, \quad\left\{T_{a}, T_{b}\right\}=\frac{1}{N_{c}} \mathbb{1} \delta_{a b}+d_{a b c} T_{c},
$$

\footnotetext{
${ }^{10}$ Summation over repeated indices is always understood.
} 
where $f_{a b c}$ is the completely antisymmetric tensor of the structure constants while $d_{a b c}$ is completely symmetric. It then holds

$$
\begin{gathered}
\operatorname{Tr}\left[T_{a} T_{b} T_{c}\right]=\frac{1}{4}\left(i f_{a b c}+d_{a b c}\right), \\
\operatorname{Tr}\left[T_{a} T_{a} T_{b} T_{b}\right]=\frac{\left(N_{c}^{2}-1\right)^{2}}{4 N_{c}}, \quad \operatorname{Tr}\left[T_{a} T_{b} T_{a} T_{b}\right]=-\frac{\left(N_{c}^{2}-1\right)}{4 N_{c}}, \\
\operatorname{Tr}\left[T_{a} T_{b} T_{c} T_{d}\right]=\frac{1}{4}\left\{\frac{1}{N_{c}} \delta_{a b} \delta_{c d}+\frac{1}{2}\left(-f_{a b e} f_{c d e}+d_{a b e} d_{c d e}+i f_{a b e} d_{c d e}+i d_{a b e} f_{c d e}\right)\right\} .
\end{gathered}
$$

Other useful identities are

$$
f_{a c e} f_{b c e}=N_{c} \delta_{a b}, \quad d_{a c e} d_{b c e}=\frac{N_{c}^{2}-4}{N_{c}} \delta_{a b},
$$

as well as $d_{\text {aae }}=0$ and $f_{\text {ace }} d_{b c e}=0$.

By defining $\gamma_{5}=\gamma_{0} \gamma_{1} \gamma_{2} \gamma_{3}$, the Euclidean anti-commutation relations

$$
\left\{\gamma_{\mu}, \gamma_{\nu}\right\}=2 \delta_{\mu \nu} \mathbb{1}
$$

imply

$$
\left\{\gamma_{5}, \gamma_{\mu}\right\}=0, \quad \gamma_{5}^{2}=\mathbb{1}, \quad \gamma_{5} \gamma_{\mu} \gamma_{5}=-\gamma_{\mu} .
$$

Useful trace identities are

$$
\operatorname{Tr}\left[\gamma_{\mu} \gamma_{\nu}\right]=4 \delta_{\mu \nu}, \quad \operatorname{Tr}\left[\gamma_{\alpha} \gamma_{\beta} \gamma_{\delta} \gamma_{\sigma}\right]=4\left[\delta_{\alpha \beta} \delta_{\delta \sigma}-\delta_{\alpha \delta} \delta_{\beta \sigma}+\delta_{\alpha \sigma} \delta_{\beta \delta}\right],
$$

and

$$
\begin{aligned}
\operatorname{Tr}\left[\gamma_{\alpha} \gamma_{\beta} \gamma_{\delta} \gamma_{\rho} \gamma_{\sigma} \gamma_{\tau}\right]= & \delta_{\alpha \beta} \operatorname{Tr}\left[\gamma_{\delta} \gamma_{\rho} \gamma_{\sigma} \gamma_{\tau}\right]-\delta_{\alpha \delta} \operatorname{Tr}\left[\gamma_{\beta} \gamma_{\rho} \gamma_{\sigma} \gamma_{\tau}\right]+\delta_{\alpha \rho} \operatorname{Tr}\left[\gamma_{\beta} \gamma_{\delta} \gamma_{\sigma} \gamma_{\tau}\right] \\
& -\delta_{\alpha \sigma} \operatorname{Tr}\left[\gamma_{\beta} \gamma_{\delta} \gamma_{\rho} \gamma_{\tau}\right]+\delta_{\alpha \tau} \operatorname{Tr}\left[\gamma_{\beta} \gamma_{\delta} \gamma_{\rho} \gamma_{\sigma}\right]
\end{aligned}
$$

while, thanks to eqs. (A.8), traces of products of an odd number of $\gamma$-matrices vanish.

\section{B Continuum theory}

In the Euclidean space-time, the path integral of QCD is defined as

$$
Z=\int D A D \bar{\psi} D \psi D \bar{c} D c e^{-S}
$$

where the integration measures on the various fields are defined as usual. The action is defined as ${ }^{11}$

$$
S=\int d^{4} x \mathcal{L}(x), \quad \mathcal{L}=\mathcal{L}^{G}+\mathcal{L}^{G F}+\mathcal{L}^{F P}+\mathcal{L}^{F}
$$

\footnotetext{
${ }^{11}$ Throughout the paper we assume the strong CP violation term to be absent.
} 
with

$$
\begin{aligned}
\mathcal{L}^{G} & =\frac{1}{2 g_{0}^{2}} \operatorname{Tr}\left[F_{\mu \nu} F_{\mu \nu}\right], \\
\mathcal{L}^{G F} & =\frac{\lambda_{0}}{g_{0}^{2}} \operatorname{Tr}\left[\partial_{\mu} A_{\mu} \partial_{\nu} A_{\nu}\right], \\
\mathcal{L}^{F P} & =-\frac{2}{g_{0}^{2}} \operatorname{Tr}\left[\bar{c} \partial_{\mu} \mathcal{D}_{\mu} c\right], \\
\mathcal{L}^{F} & =\bar{\psi}\left[\gamma_{\mu} D_{\mu}+M_{0}\right] \psi,
\end{aligned}
$$

where $g_{0}$ is the bare coupling constant, $\lambda_{0}$ is the gauge-fixing parameter, the trace is over the color index and

$$
\begin{aligned}
F_{\mu \nu} & =\partial_{\mu} A_{\nu}-\partial_{\nu} A_{\mu}-i\left[A_{\mu}, A_{\nu}\right], \quad A_{\mu}=A_{\mu}^{a} T^{a} \\
D_{\mu} & =\partial_{\mu}-i A_{\mu}, \\
\mathcal{D}_{\mu} c & =\partial_{\mu} c-i\left[A_{\mu}, c\right], \quad c=c^{a} T^{a} .
\end{aligned}
$$

The quark and anti-quark fields, $\psi$ and $\bar{\psi}$ have $N_{f}$-flavour components $\psi^{f}, \bar{\psi}^{f}, f=$ $1, \ldots, N_{f}$ and, accordingly, the mass matrix $M_{0}=\operatorname{diag}\left(m_{0,1}, m_{0,2}, m_{0,3}, \ldots\right)$ is a $N_{f} \times N_{f}$ matrix, whose entries on the diagonal are the bare quark masses. It turns out to be useful also to define

$$
\overleftrightarrow{D}_{\mu}=D_{\mu}-\overleftarrow{D}_{\mu}, \quad \overleftarrow{D}_{\mu}=\overleftarrow{\partial}_{\mu}+i A_{\mu}
$$

\section{B.1 Dimensional regularization}

Here we report the essential formulas of dimensional regularization which are needed in this paper. We follow the conventions of ref. [25], see also ref. [41] for a recent review. By replacing $\int d^{4} x \rightarrow \int d^{D} x$, one defines the renormalized coupling $g$ and quark mass matrix $M$ as

$$
\begin{aligned}
g_{0}^{2} & =\mu^{2 \epsilon} g^{2} \mathcal{Z}_{g}^{-1}, \\
M_{0} & =M \mathcal{Z}_{m}^{-1}
\end{aligned}
$$

where $D=4-2 \epsilon$ and $\mu$ is a mass parameter. A generic renormalization constant, including those of composite operators, is expanded in $g^{2}$. In the MS scheme is then implicitly fixed by requiring it to be a polynomial in $1 / \epsilon$ with no constant term

$$
\mathcal{Z}=1+\sum_{k=1}^{\infty} \mathcal{Z}^{(k)} g^{2 k}, \quad \mathcal{Z}^{(k)}=\sum_{j=1}^{k} \mathcal{Z}^{(k, j)} \frac{1}{\epsilon^{j}} .
$$

The $\beta$-function of the theory is defined as

$$
\beta(\epsilon, g)=\mu \frac{\partial g}{\partial \mu}=-\epsilon g\left\{1-\frac{g}{2} \frac{\partial}{\partial g} \ln Z_{g}\right\}^{-1}=-\epsilon g+\beta(g)
$$

where

$$
\beta(g)=-g^{3} \sum_{k=0}^{\infty} b_{k} g^{2 k}
$$


with the first coefficient given by

$$
b_{0}=\frac{1}{(4 \pi)^{2}}\left\{\frac{11}{3} N_{c}-\frac{2}{3} N_{f}\right\} .
$$

The anomalous dimension of the quark mass is defined as

$$
\gamma_{m}=\beta(\epsilon, g) \frac{\partial}{\partial g} \ln \mathcal{Z}_{m}=-g^{2} \sum_{k=0}^{\infty} d_{k} g^{2 k},
$$

where the first coefficient is

$$
d_{0}=\frac{3}{(4 \pi)^{2}} \frac{N_{c}^{2}-1}{N_{c}}
$$

\section{Lattice theory}

The action of the lattice theory reads

$$
S=S^{G}+S^{F}
$$

where $S^{G}$ and $S^{F}$ are the gluonic and fermionic contributions respectively. For the gluonic one we consider the Wilson plaquette action

$$
S^{G}=\frac{1}{g_{0}^{2}} \sum_{x} \sum_{\mu, \nu} \operatorname{Re} \operatorname{Tr}\left\{\mathbb{1}-U_{\mu \nu}(x)\right\},
$$

where $g_{0}$ is the bare gauge coupling. The plaquette field is defined by

$$
U_{\mu \nu}(x)=U_{\mu}(x) U_{\nu}(x+a \hat{\mu}) U_{\mu}^{\dagger}(x+a \hat{\nu}) U_{\nu}^{\dagger}(x),
$$

where $\hat{\mu}, \hat{\nu}$ are unit vectors oriented along the directions $\mu, \nu$ respectively. The fermionic part reads

$$
S^{F}=a^{4} \sum_{x} \bar{\psi}(x)\left(D+M_{0}\right) \psi(x),
$$

where $M_{0}$ is the bare quark mass matrix, and for $D$ we choose the $\mathrm{O}(a)$-improved WilsonDirac operator

$$
D=D_{\mathrm{w}}+a D_{\mathrm{sw}} .
$$

The first operator on the r.h.s. is the massless Wilson-Dirac operator defined by

$$
D_{\mathrm{w}}=\frac{1}{2}\left\{\gamma_{\mu}\left(\nabla_{\mu}^{*}+\nabla_{\mu}\right)-a \nabla_{\mu}^{*} \nabla_{\mu}\right\},
$$

where $\nabla_{\mu}^{*}, \nabla_{\mu}$ are covariant lattice derivatives acting on the quark fields as follows

$$
\begin{aligned}
& a \nabla_{\mu} \psi(x)=U_{\mu}(x) \psi(x+a \hat{\mu})-\psi(x), \\
& a \nabla_{\mu}^{*} \psi(x)=\psi(x)-U_{\mu}^{\dagger}(x-a \hat{\mu}) \psi(x-a \hat{\mu}) .
\end{aligned}
$$


The second term is the Sheikholeslami-Wohlert operator defined by [15]

$$
D_{\mathrm{sw}} \psi(x)=c_{\mathrm{sw}}\left(g_{0}\right) \frac{1}{4} \sigma_{\mu \nu} \widehat{F}_{\mu \nu}(x) \psi(x),
$$

where $\sigma_{\mu \nu}=\frac{i}{2}\left[\gamma_{\mu}, \gamma_{\nu}\right], \widehat{F}_{\mu \nu}(x)$ is the clover discretization of the field strength tensor ${ }^{12}$

$$
\widehat{F}_{\mu \nu}(x)=\frac{i}{8 a^{2}}\left\{Q_{\mu \nu}(x)-Q_{\nu \mu}(x)\right\},
$$

and

$$
\begin{aligned}
Q_{\mu \nu}(x)= & U_{\mu}(x) U_{\nu}(x+a \hat{\mu}) U_{\mu}^{\dagger}(x+a \hat{\nu}) U_{\nu}^{\dagger}(x) \\
& +U_{\nu}(x) U_{\mu}^{\dagger}(x-a \hat{\mu}+a \hat{\nu}) U_{\nu}^{\dagger}(x-a \hat{\mu}) U_{\mu}(x-a \hat{\mu}) \\
& +U_{\mu}^{\dagger}(x-a \hat{\mu}) U_{\nu}^{\dagger}(x-a \hat{\mu}-a \hat{\nu}) U_{\mu}(x-a \hat{\mu}-a \hat{\nu}) U_{\nu}(x-a \hat{\nu}) \\
& +U_{\nu}^{\dagger}(x-a \hat{\nu}) U_{\mu}(x-a \hat{\nu}) U_{\nu}(x+a \hat{\mu}-a \hat{\nu}) U_{\mu}^{\dagger}(x) .
\end{aligned}
$$

The coefficient $c_{\mathrm{sw}}$ is tuned in order to remove $\mathrm{O}(a)$ lattice artifacts generated by the action in on-shell correlation functions $[15,31]$. The gauge-invariant path integral is

$$
Z=\int D U D \bar{\psi} D \psi e^{-S}
$$

It is also useful to define

$$
\overleftrightarrow{\nabla}_{\mu}=\nabla_{\mu}-\overleftarrow{\nabla}_{\mu}, \quad \overleftrightarrow{\nabla}_{\mu}^{*}=\nabla_{\mu}^{*}-\overleftarrow{\nabla}_{\mu}^{*}
$$

with $\nabla_{\mu}, \nabla_{\mu}^{*}$ being the lattice covariant derivatives in eq. (C.7), and

$$
\begin{aligned}
a \bar{\psi}(x) \overleftarrow{\nabla}_{\mu} & =\bar{\psi}(x+a \hat{\mu}) U_{\mu}^{\dagger}(x)-\bar{\psi}(x) \\
a \bar{\psi}(x) \overleftarrow{\nabla}_{\mu}^{*} & =\bar{\psi}(x)-\bar{\psi}(x-a \hat{\mu}) U_{\mu}(x-a \hat{\mu})
\end{aligned}
$$

\section{Automatic $\mathrm{O}(a)$-improvement with massless quarks}

In the absence of spontaneous chiral symmetry breaking, chirally symmetric correlators of Wilson fermions in the presence of an even number of massless quarks are proven to be automatically $\mathrm{O}(a)$-improved $[42,43]$. This occurs, for instance, in a finite volume without boundaries [43] or in the thermal theory at high temperature [44]. To extend this result to a generic number of flavours $N_{f}>1$ but still small enough to have asymptotic freedom, we consider the discrete axial symmetry $S_{5}$ defined as

$$
\psi \rightarrow \psi^{\prime}=e^{i \frac{\pi}{N_{f}} \gamma_{5}} \psi, \quad \bar{\psi} \rightarrow \bar{\psi}^{\prime}=\bar{\psi} e^{i \frac{\pi}{N_{f}} \gamma_{5}} .
$$

This non-anomalous element of the $\mathrm{U}(1)_{A}$ group indeed allows for a simple generalization of the line of argumentation given in refs. [42, 43]. In the massless limit, the action of the Symanzik's effective continuum theory reads

$$
S_{\text {eff }}=S_{0}+a S_{1}+\mathrm{O}\left(a^{2}\right),
$$

\footnotetext{
${ }^{12}$ Notice that for historical reasons the field strength tensor discretization $\widehat{F}_{\mu \nu}$ adopted in eq. (C.8) is not traceless at variance of the one used to define the energy-momentum tensor, see eq. (3.3).
} 
where $S_{0}$ is defined as in eq. (B.2) but with the bare coupling replaced by the renormalized one, and

$$
S_{1}=c_{1} \int d^{4} x \bar{\psi}(x) \sigma_{\mu \nu} F_{\mu \nu}(x) \psi(x) .
$$

The leading discretization effects in the connected correlation function of a multi-local renormalized field $O$ are then given by the corresponding continuum correlation functions with the insertion either of $S_{1}$ or of the $\mathrm{O}(a)$-counterterm $\delta O$ for the field $O$,

$$
\langle O\rangle_{\text {con }}^{\text {lat }}=\langle O\rangle_{\text {con }}-a\left\langle S_{1} O\right\rangle_{\text {con }}+a\langle\delta O\rangle_{\text {con }}+\mathrm{O}\left(a^{2}\right) .
$$

If we restrict ourselves to fields $O_{\text {inv }}$ invariant under parity and the chiral symmetry $S_{5}$, such as (the fermionic component of) $T_{\mu \nu}$ in eq. (2.1) in the massless limit, the invariance of the measure and of the action $S_{0}$ implies

$$
\left[1-\cos \left(\frac{2 \pi}{N_{f}}\right)\right]\left\langle S_{1} O_{\text {inv }}\right\rangle_{\text {con }}=0
$$

when spontaneous chiral symmetry breaking is absent. Moreover $\langle\delta O\rangle_{\text {con }}=0$ as well because fields with the same quantum numbers as $O_{\mathrm{inv}}$ but of one dimension higher are not invariant under the symmetry $S_{5}$ since they must have and extra derivative and therefore an extra $\gamma$-matrix with respect to $O_{\text {inv }}$, a fact that was essential to prove the automatic $\mathrm{O}(a)$-improvement in the twisted mass QCD regularization [42]. The eq. (D.4) then reads

$$
\left\langle O_{\text {inv }}\right\rangle_{\text {con }}^{\text {lat }}=\left\langle O_{\text {inv }}\right\rangle_{\text {con }}+\mathrm{O}\left(a^{2}\right) .
$$

The very same conclusion can be reached by using the $R_{5}$ discrete symmetry in ref. [42] for $N_{f}=2$, and an element of the axial subgroup $Z_{N_{f}}$ of the non-Abelian chiral symmetry group for larger $N_{f}$.

\section{E Propagators and vertices for perturbation theory}

On the lattice, perturbation theory is normally set-up in terms of algebra-valued fields $A_{\mu}(x)$ defined as

$$
U_{\mu}(x)=e^{-i a g_{0} A_{\mu}(x)}=1-i a g_{0} A_{\mu}(x)+\ldots, \quad A_{\mu}(x)=A_{\mu}^{a}(x) T_{a},
$$

where we opt for a minus sign in the exponential so to recover, in the naive continuum limit, the widely used conventions in appendix B. By inserting (E.1) in the expressions in appendix $\mathrm{C}$ and by expanding to the appropriate order, the analogous continuum formulas given in appendix $\mathrm{B}$ are recovered after the usual field rescaling $A_{\mu}(x) \rightarrow A_{\mu}(x) / g_{0}$. The free theory in recovered in the limit $g_{0} \rightarrow 0$.

In the presence of the boundary conditions (3.14), the Fourier transform can be written $\operatorname{as}^{13}$

$$
A_{\mu}(x)=\int_{p_{\boldsymbol{\xi}}} A_{\mu}(p) e^{i p\left(x+\frac{a}{2} \hat{\mu}\right)},
$$

\footnotetext{
${ }^{13}$ To avoid burdening the notation, we use the same symbol for the field and for its Fourier transform since any ambiguity is resolved from the context.
} 
where, for a generic function $f(p)$, the finite-volume integration is defined to be

$$
\int_{p_{\boldsymbol{\xi}}} f(p)=\frac{1}{L_{0} L_{1} L_{2} L_{3}} \sum_{n} f(p)
$$

with

$$
p_{0}=\frac{2 \pi n_{0}}{L_{0}}-\sum_{k=1}^{3} p_{k} \xi_{k}, \quad p_{k}=\frac{2 \pi n_{k}}{L_{k}}
$$

and $n_{\mu}=0, \ldots, L_{\mu} / a-1$. For lattice fermions satisfying the boundary conditions in eqs. (2.7) and (2.58), the Fourier transform can be written as

$$
\psi(x)=\int_{p_{\boldsymbol{\xi}, \theta}} \psi(p) e^{i p x}, \quad \bar{\psi}(x)=\int_{p_{\boldsymbol{\xi}, \theta}} \bar{\psi}(p) e^{-i p x},
$$

where the integration $\int_{p_{\xi, \theta}}$ is defined as in eq. (E.3) but for the set of lattice momenta

$$
p_{0}=\frac{2 \pi n_{0}}{L_{0}}+\frac{\theta_{0}}{L_{0}}+\frac{\pi}{L_{0}}-\sum_{k=1}^{3} p_{k} \xi_{k}, \quad p_{k}=\frac{2 \pi n_{k}}{L_{k}}+\frac{\theta_{k}}{L_{k}} .
$$

The term $\pi / L_{0}$ in $p_{0}$ is due to the anti-periodicity of the boundary conditions along the temporal direction.

When $L_{k} \rightarrow \infty$ for all three spatial directions $k$, the integration in eq. (E.3) becomes

$$
\int_{p_{\boldsymbol{\xi}}} f(p) \rightarrow \frac{1}{L_{0}} \sum_{n_{0}} \int_{B Z} \frac{d^{3} \boldsymbol{p}}{(2 \pi)^{3}} f(p),
$$

where $B Z$ stands for the Brillouin zone. The analogous holds for the fermion integrals (E.5) which become independent on $\theta_{k}$. If also $L_{0} \rightarrow \infty$, the sum over $n_{0}$ in eq. (E.7) is replaced by the integral as well, which becomes independent on the shift $\boldsymbol{\xi}$ and the twist $\theta_{0}$.

As expected by general quantum field theory arguments, the expressions of the propagators and of the vertices of the theory with shifted and twisted boundary conditions are equal to those valid for periodic boundary conditions provided the definition of the momenta are replaced by those in eqs. (E.4) and (E.6), i.e. $\boldsymbol{\xi}$ and $\theta$ enter the values of the allowed momenta only. For consistency and to define our conventions, however, we report their definitions in the rest of this appendix.

\section{E.1 Gluon propagator}

By adding the gauge-fixing contribution ${ }^{14}$

$$
S^{G F}=a^{4} \sum_{x} \operatorname{Tr}\left[\left(\partial_{\mu}^{*} A_{\mu}\right)(x)\left(\partial_{\mu}^{*} A_{\nu}\right)(x)\right]
$$

to the gluonic action (C.2), the free-gluon propagator in Feynman gauge reads

$$
\left\langle A_{\mu}^{a}(x) A_{\nu}^{b}(y)\right\rangle_{\boldsymbol{\xi}}=\int_{p_{\boldsymbol{\xi}}} D_{\mu \nu}^{a b}(p) e^{i p(x-y)}
$$

where

$$
D_{\mu \nu}^{a b}(p)=\frac{\delta_{a b} \delta_{\mu \nu}}{D_{G}(p)}, \quad D_{G}(p)=\sum_{\mu=0}^{3} \hat{p}_{\mu}^{2}, \quad \hat{p}_{\mu}=\frac{2}{a} \sin \left(\frac{a p_{\mu}}{2}\right) .
$$

\footnotetext{
${ }^{14}$ As usual the backward lattice derivative $\partial_{\mu}^{*}$ is defined as eq. (C.7) but with the link omitted.
} 


\section{E.2 Ghost propagator}

By following the usual Faddeev-Popov (FP) procedure, we add to the gluonic action the contribution coming from the FP determinant

$$
S^{F P}=-2 a^{4} \sum_{x} \operatorname{Tr}\left[\bar{c}(x) \partial_{\mu}^{*} \widehat{D}_{\mu} c(x)\right]
$$

where

$$
\widehat{D}_{\mu} c=\left[M\left(A_{\mu}\right)\right]^{-1} \nabla_{\mu} c-i g_{0}\left[A_{\mu}, c\right], \quad M\left(A_{\mu}\right)=\left(\frac{1-e^{-i \Phi\left(A_{\mu}\right)}}{i \Phi\left(A_{\mu}\right)}\right),
$$

and $\Phi\left(A_{\mu}\right)$ is a matrix in the adjoint representation of the algebra of $\mathrm{SU}\left(N_{c}\right)$ whose matrix elements are $\left[\Phi\left(A_{\mu}\right)\right]^{a b}=i a g_{0} f^{a b c} A_{\mu}^{c}$. The ghost propagator then reads

$$
\left\langle c^{a}(x) \bar{c}^{b}(y)\right\rangle_{\boldsymbol{\xi}}=\int_{p_{\boldsymbol{\xi}}} \Delta_{G}^{a b}(p) e^{i p(x-y)}, \quad \Delta_{G}(p)=\frac{\delta_{a b}}{D_{G}(p)} .
$$

Notice that the ghost and anti-ghost fields satisfy the same boundary conditions of the gauge field.

\section{E.3 Fermion propagator}

The free-fermion propagator for a single flavour is given by

$$
\langle\psi(x) \bar{\psi}(y)\rangle_{\boldsymbol{\xi}, \theta}=\int_{p_{\boldsymbol{\xi}, \theta}} S(p) e^{i p(x-y)},
$$

where

$$
S(p)=\frac{-i \gamma_{\mu} \bar{p}_{\mu}+m_{0}(p)}{D_{F}(p)}
$$

and

$$
D_{F}(p)=\sum_{\mu=0}^{3} \bar{p}_{\mu}^{2}+m_{0}^{2}(p), \quad m_{0}(p)=m_{0}+\frac{a}{2} \sum_{\mu=0}^{3} \hat{p}_{\mu}^{2}, \quad \bar{p}_{\mu}=\frac{1}{a} \sin \left(a p_{\mu}\right) .
$$

\section{E.4 Gluonic interaction}

The perturbative expansion of the Wilson action (C.2) can be written as

$$
S^{G}=S^{G, 0}+g_{0} S^{G, 1}+g_{0}^{2} S^{G, 2}+\mathrm{O}\left(g_{0}^{3}\right),
$$

where $S^{G, 0}$ is the tree-level gluonic action, while the contributions from the three- and the four-gluon vertices are

$$
\begin{aligned}
S^{G, 1}= & \frac{i}{6} f_{a b c} \int_{k_{\boldsymbol{\xi}} ; p_{\boldsymbol{\xi}} ; q_{\boldsymbol{\xi}}} \bar{\delta}(k+p+q) A_{\mu}^{a}(k) A_{\nu}^{b}(p) A_{\lambda}^{c}(q) \\
& \cdot\left[\delta_{\lambda \nu}(\widehat{q-p})_{\mu} \mathrm{c}_{\nu}\left(\frac{k}{2}\right)+\delta_{\mu \lambda}(\widehat{k-q})_{\nu} \mathrm{c}_{\mu}\left(\frac{p}{2}\right)+\delta_{\mu \nu}(\widehat{p-k})_{\lambda} \mathrm{c}_{\mu}\left(\frac{q}{2}\right)\right], \\
S^{G, 2}= & \frac{-1}{24} \int_{k_{\boldsymbol{\xi}} ; q_{\xi} ; r_{\boldsymbol{\xi}} ; s_{\boldsymbol{\xi}}} \bar{\delta}(k+q+r+s) A_{\mu}^{a}(k) A_{\nu}^{b}(q) A_{\lambda}^{c}(r) A_{\rho}^{d}(s) \\
& \cdot\left[\mathcal{X}_{\mu \nu \lambda \rho}^{a b c d}(k, q, r, s)+\mathcal{Y}_{\mu \nu \lambda \rho}^{a b c d}(k, q, r, s)\right]
\end{aligned}
$$


with

$$
\begin{aligned}
& \mathcal{X}_{\mu \nu \lambda \rho}^{a b c d}(k, q, r, s)=-f_{a b e} f_{c d e}\left\{\delta_{\mu \lambda} \delta_{\nu \rho}\left[\mathrm{c}_{\mu}\left(\frac{q-s}{2}\right) \mathrm{c}_{\nu}\left(\frac{k-r}{2}\right)-\frac{a^{4}}{12} \hat{k}_{\nu} \hat{q}_{\mu} \hat{r}_{\nu} \hat{s}_{\mu}\right]\right. \\
& -\delta_{\mu \rho} \delta_{\nu \lambda}\left[\mathrm{c}_{\mu}\left(\frac{q-r}{2}\right) \mathrm{c}_{\nu}\left(\frac{k-s}{2}\right)-\frac{a^{4}}{12} \hat{k}_{\nu} \hat{q}_{\mu} \hat{r}_{\mu} \hat{s}_{\nu}\right]+\frac{a^{2}}{6}\left[\delta_{\nu \lambda} \delta_{\nu \rho}(\widehat{s-r})_{\mu} \hat{k}_{\nu} \mathrm{c}_{\mu}\left(\frac{q}{2}\right) \quad \mathrm{E} .\right. \\
& \left.-\delta_{\mu \lambda} \delta_{\mu \rho}(\widehat{s-r})_{\nu} \hat{q}_{\mu} \mathrm{c}_{\nu}\left(\frac{k}{2}\right)+\delta_{\mu \nu} \delta_{\mu \rho}(\widehat{q-k})_{\lambda} \hat{r}_{\rho} \mathrm{c}_{\lambda}\left(\frac{s}{2}\right)-\delta_{\mu \nu} \delta_{\mu \lambda}(\widehat{q-k})_{\rho} \hat{s}_{\lambda} \mathrm{c}_{\rho}\left(\frac{r}{2}\right)\right] \\
& \left.+\frac{a^{2}}{12} \delta_{\mu \nu} \delta_{\mu \lambda} \delta_{\mu \rho} \sum_{\sigma}(\widehat{q-k})_{\sigma}(\widehat{s-r})_{\sigma}\right\}+(b \leftrightarrow c, \nu \leftrightarrow \lambda, q \leftrightarrow r)+(b \leftrightarrow d, \nu \leftrightarrow \rho, q \leftrightarrow s), \\
& \mathcal{Y}_{\mu \nu \lambda \rho}^{a b c d}(k, q, r, s)=\frac{a^{4}}{12}\left\{\frac{2}{N}\left(\delta_{a b} \delta_{c d}+\delta_{a c} \delta_{b d}+\delta_{a d} \delta_{b c}\right)+\left(d_{a b e} d_{c d e}+d_{a c e} d_{b d e}+d_{a d e} d_{b c e}\right)\right\} . \\
& \cdot\left\{\delta_{\mu \nu} \delta_{\mu \lambda} \delta_{\mu \rho} \sum_{\sigma} \hat{k}_{\sigma} \hat{q}_{\sigma} \hat{r}_{\sigma} \hat{s}_{\sigma}-\delta_{\mu \nu} \delta_{\mu \lambda} \hat{k}_{\rho} \hat{q}_{\rho} \hat{r}_{\rho} \hat{s}_{\mu}-\delta_{\mu \nu} \delta_{\mu \rho} \hat{k}_{\lambda} \hat{q}_{\lambda} \hat{s}_{\lambda} \hat{r}_{\mu}-\delta_{\mu \lambda} \delta_{\mu \rho} \hat{k}_{\nu} \hat{r}_{\nu} \hat{s}_{\nu} \hat{q}_{\mu}\right. \\
& \left.\quad-\delta_{\nu \lambda} \delta_{\nu \rho} \hat{q}_{\mu} \hat{r}_{\mu} \hat{s}_{\mu} \hat{k}_{\nu}+\delta_{\mu \nu} \delta_{\lambda \rho} \hat{k}_{\lambda} \hat{q}_{\lambda} \hat{r}_{\mu} \hat{s}_{\mu}+\delta_{\mu \lambda} \delta_{\nu \rho} \hat{k}_{\nu} \hat{r}_{\nu} \hat{q}_{\mu} \hat{s}_{\mu}+\delta_{\mu \rho} \delta_{\nu \lambda} \hat{k}_{\nu} \hat{s}_{\nu} \hat{q}_{\mu} \hat{r}_{\mu}\right\}, \quad(\mathrm{E} .21
\end{aligned}
$$

$\mathrm{c}_{\mu}(p)=\cos \left(a p_{\mu}\right)$ and

$$
\bar{\delta}(p)=(2 \pi)^{4} \delta^{(4)}(p)=a^{4} \sum_{x} e^{i p x} .
$$

The Jacobian from the Haar integration measure due to the change of variables (E.1) can be recast in the form of an extra contribution to the action which reads

$$
S^{M}=g_{0}^{2} S^{M, 2}+\mathrm{O}\left(g_{0}^{4}\right), \quad S^{M, 2}=\frac{N}{24 a^{2}} \delta_{a b} \delta_{\mu \nu} \int_{k_{\xi} ; q_{\boldsymbol{\xi}}} \bar{\delta}(k+q) A_{\mu}^{a}(k) A_{\nu}^{b}(p) .
$$

\section{E.5 Ghost-gluon interaction}

The expansion of the FP action (E.11) reads

$$
S^{F P}=S^{F P, 0}+g_{0} S^{F P, 1}+g_{0}^{2} S^{F P, 2}+\mathrm{O}\left(g_{0}^{3}\right)
$$

where $S^{F P, 0}$ is the tree-level term, while

$$
\begin{aligned}
S^{F P, 1}= & i f_{a b c} \int_{k_{\xi} ; p_{\xi} ; q_{\xi}} \bar{\delta}(p+q-k) \bar{c}^{a}(p) A_{\mu}^{b}(q) c^{c}(k)\left[\hat{k}_{\mu} \mathrm{c}_{\mu}\left(\frac{p}{2}\right)\right], \\
S^{F P, 2}= & -\delta_{\mu \nu} \frac{a^{2}}{24}\left(f_{a b e} f_{d c e}+f_{a c e} f_{d b e}\right) \\
& \times \int_{k_{\boldsymbol{\xi}} ; q_{\boldsymbol{\xi}} ; r_{\boldsymbol{\xi}} ; s_{\boldsymbol{\xi}}} \bar{\delta}(k+q+r-s) \bar{c}^{a}(r) A_{\mu}^{b}(q) A_{\nu}^{c}(k) c^{d}(s) \hat{r}_{\mu} \hat{s}_{\mu} .
\end{aligned}
$$

\section{E.6 Quark-gluon interaction}

By inserting eq. (E.1) into eq. (C.4) and by expanding in $g_{0}$, the fermionic action reads

$$
S^{F}=S^{F, 0}+g_{0} S^{F, 1}+g_{0}^{2} S^{F, 2}+g_{0} S^{S W, 1}+g_{0}^{2} S^{S W, 2}+\mathrm{O}\left(g_{0}^{3}\right),
$$


where $S^{F, 0}$ is the tree-level contribution, while

$$
\begin{aligned}
S^{F, 1}= & -\sum_{\mu} \int_{q_{\boldsymbol{\xi}} ; p_{\boldsymbol{\xi}, \theta} ; r_{\boldsymbol{\xi}, \theta}} \bar{\delta}(p-q-r) \bar{\psi}(p) A_{\mu}(q) \\
& \times\left[i \gamma_{\mu} \mathrm{c}_{\mu}\left(\frac{p+r}{2}\right)+\frac{a}{2}(\widehat{p+r})_{\mu}\right] \psi(r), \\
S^{F, 2}= & \frac{a}{2} \sum_{\mu} \int_{q_{\boldsymbol{\xi}} ; r_{\boldsymbol{\xi}} ; p_{\boldsymbol{\xi}, \theta} ; s_{\boldsymbol{\xi}, \theta}} \bar{\delta}(p-q-r-s) \bar{\psi}(p) A_{\mu}(q) A_{\mu}(r) \\
& \times\left[\mathrm{c}_{\mu}\left(\frac{p+s}{2}\right)-i \frac{a}{2} \gamma_{\mu}(\widehat{p+s})_{\mu}\right] \psi(s),
\end{aligned}
$$

and

$$
S^{S W, 1}=-i \frac{a}{2} c_{\mathrm{SW}} \sum_{\mu, \nu} \int_{q_{\boldsymbol{\xi}} ; p_{\boldsymbol{\xi}, \theta} ; r_{\boldsymbol{\xi}, \theta}} \bar{\delta}(p-q-r) \bar{\psi}(p) A_{\mu}(q) \sigma_{\mu \nu} \psi(r)\left[\bar{q}_{\nu} \mathrm{c}_{\mu}\left(\frac{q}{2}\right)\right]
$$

The $S^{S W, 2}$ has two quark and two gluonic lines, but it does not contribute to the quantities we are interested in this paper due to its color structure.

\section{F The free energy density}

In this appendix we report the coefficients of the 1-loop perturbative expansion of the freeenergy density defined in eqs. (5.1)-(5.3). The gluonic and fermionic tree-level values are

$$
f^{G(0)}=\int_{p_{\boldsymbol{\xi}}} \ln \left[a^{2} D_{G}(p)\right], \quad f^{F(0)}=-2 \int_{p_{\boldsymbol{\xi}, \theta}} \ln \left[a^{2} D_{F}(p)\right],
$$

and the derivative of the fermionic contribution with respect to the bare mass is

$$
\frac{\partial f^{F(0)}}{\partial m_{0}}=-4 F^{(8)}
$$

where $F^{(8)}$ is defined in appendix J. The 1-loop contributions result from connected diagrams with no external legs. The gluonic ones are

$$
\left\langle S^{G, 2}+S^{F P, 2}+S^{M, 2}-\frac{\left(S^{G, 1}\right)^{2}+\left(S^{F P, 1}\right)^{2}}{2}\right\rangle_{\mathrm{con}}=\left(N_{c}^{2}-1\right)\left[N_{c} f^{G\left(1, N_{c}\right)}+\frac{1}{N_{c}} f^{G\left(1, \frac{1}{N_{c}}\right)}\right]
$$

from which we have

$$
\begin{aligned}
& f^{G\left(1, N_{c}\right)}=\left\{\left(B^{(0)}\right)^{2}-\frac{1}{2} \sum_{\sigma}\left[B^{(0)}-B_{\sigma}^{(3)}\right]^{2}+\frac{1}{2} a^{2} K_{1}+\frac{1}{24} a^{4} K_{2}-\frac{1}{2 a^{2}} B^{(0)}\right\}, \\
& f^{G\left(1, \frac{1}{N_{c}}\right)}=\frac{1}{2}\left\{\sum_{\sigma}\left[B^{(0)}-B_{\sigma}^{(3)}\right]^{2}+\frac{1}{8 a^{4}}\right\}
\end{aligned}
$$


where

$$
\begin{aligned}
K_{1} & =\int_{p_{\xi} ; q_{\xi} ; k_{\xi}} \frac{\bar{\delta}(p+q+k)}{D_{G}(p) D_{G}(q) D_{G}(k)} \sum_{\mu} \hat{p}_{\mu}^{2} \hat{q}_{\mu}^{2}, \\
K_{2} & =\int_{p_{\xi} ; q_{\xi} ; k_{\xi}} \frac{\bar{\delta}(p+q+k)}{D_{G}(p) D_{G}(q) D_{G}(k)} \sum_{\mu} \hat{p}_{\mu}^{2} \hat{q}_{\mu}^{2} \hat{k}_{\mu}^{2} .
\end{aligned}
$$

The fermionic contribution is

$$
\left\langle S^{F, 2}-\frac{\left(S^{F, 1}\right)^{2}}{2}\right\rangle_{\mathrm{con}}=\left(N_{c}^{2}-1\right) N_{f} f^{F\left(1, N_{f}\right)},
$$

where

$$
\begin{aligned}
& f^{F\left(1, N_{f}\right)}=B^{(0)}\left[\frac{1}{a^{2}}-a\left(a m_{0}+4\right) F^{(8)}\right]+\int_{q_{\boldsymbol{\xi}} ; p_{\boldsymbol{\xi}, \theta} ; k_{\boldsymbol{\xi}, \theta}} \frac{\bar{\delta}(p-q-k)}{D_{G}(q) D_{F}(p) D_{F}(k)} \\
& \times\left[a m_{0}(k) \sum_{\sigma} \bar{r}_{\sigma} \bar{p}_{\sigma}+a m_{0}(p) \sum_{\sigma} \bar{k}_{\sigma} \bar{r}_{\sigma}-m_{0}(k) m_{0}(p) \sum_{\sigma} \mathrm{c}_{\sigma}(r)+\sum_{\sigma} \bar{p}_{\sigma} \bar{k}_{\sigma}\left(\mathrm{c}_{\sigma}(r)-3\right)\right],
\end{aligned}
$$

and $r=p+k$.

\section{F.1 $\mathrm{O}(a)$-improved action}

The insertion of the improvement term modifies only $f^{F\left(1, N_{f}\right)}$ so that

$$
f^{F\left(1, N_{f}\right)} \longrightarrow f^{F\left(1, N_{f}\right)}+\mathcal{F}^{F 1}+\mathcal{F}^{F 2}
$$

where

$$
\begin{gathered}
\left\langle\left(-S^{S W, 1} S^{F, 1}\right)\right\rangle_{\text {con }}=\left(N_{c}^{2}-1\right) N_{f} \mathcal{F}^{F 1}, \\
\left\langle-\frac{1}{2}\left(S^{S W, 1}\right)^{2}\right\rangle_{\text {con }}=\left(N_{c}^{2}-1\right) N_{f} \mathcal{F}^{F 2} .
\end{gathered}
$$

The expressions of $\mathcal{F}^{F 1}$ and $\mathcal{F}^{F 2}$ in terms of the integrals defined in appendix $\mathrm{J}$ are

$$
\begin{aligned}
\mathcal{F}^{F 1}=-\frac{a c_{\mathrm{sw}}}{2} \int_{q_{\boldsymbol{\xi}} ; p_{\boldsymbol{\xi}, \theta} ; k_{\boldsymbol{\xi}, \theta}} & \frac{\bar{\delta}(p-q-k)}{D_{G}(q) D_{F}(p) D_{F}(k)}\left\{a \sum_{\sigma \rho}\left[\left(\bar{p}_{\rho}+\bar{k}_{\rho}\right) \bar{q}_{\sigma}\left(\bar{p}_{\sigma} \bar{k}_{\rho}-\bar{k}_{\sigma} \bar{p}_{\rho}\right)\right]\right. \\
& \left.-\sum_{\sigma}\left\{\bar{q}_{\sigma}\left[m_{0}(k) \bar{p}_{\sigma}-m_{0}(p) \bar{k}_{\sigma}\right] \sum_{\rho \neq \sigma}\left[\mathrm{c}_{\rho}(p)+\mathrm{c}_{\rho}(k)\right]\right\}\right\},
\end{aligned}
$$

and

$$
\begin{gathered}
\mathcal{F}^{F 2}=\frac{a^{2} c_{\mathrm{Sw}}^{2}}{8} \int_{q_{\boldsymbol{\xi}} ; p_{\boldsymbol{\xi}, \theta} ; k_{\boldsymbol{\xi}, \theta}} \frac{\bar{\delta}(p-q-k)}{D_{G}(q) D_{F}(p) D_{F}(k)}\left\{m_{0}(p) m_{0}(k) \sum_{\sigma}\left\{\bar{q}_{\sigma}^{2}\left[3+\sum_{\rho \neq \sigma} \mathrm{c}_{\rho}(q)\right]\right\}\right. \\
\left.+2 \sum_{\sigma \rho} \bar{k}_{\sigma} \bar{q}_{\sigma} \bar{p}_{\rho} \bar{q}_{\rho}\left(2-\mathrm{c}_{\sigma}(q)+\sum_{\lambda \neq \rho} \mathrm{c}_{\lambda}(q)\right)-\sum_{\sigma \rho} \bar{k}_{\sigma} \bar{p}_{\sigma} \bar{q}_{\rho}^{2}\left(1-2 \mathrm{c}_{\sigma}(q)+\sum_{\lambda \neq \rho} \mathrm{c}_{\lambda}(q)\right)\right\} .
\end{gathered}
$$

As an explicit check of the whole computation, we compared the derivative of the free-energy density with respect to $\theta_{\mu}$ to the expectation value of the $\mu$-component of the conserved vector current (3.19), e.g. for $\mu=0$ this corresponds to verify the lattice analog of eq. (2.12). The required 1-loop computation of the expectation value of the vector current is reported in the following appendix. 


\section{G Expectation value of the vector current}

By expanding the conserved current in eq. (3.19) to order $g_{0}^{2}$

$$
V_{\mu}^{c}=V_{\mu}^{(0)}+g_{0} V_{\mu}^{(1)}+g_{0}^{2} V_{\mu}^{(2)}+\mathcal{O}\left(g_{0}^{3}\right),
$$

its expectation value at one loop can be written as

$$
\left\langle V_{\mu}^{c}\right\rangle=\mathcal{V}_{\mu}^{(0)}+g_{0}^{2} \mathcal{V}_{\mu}^{(1)}
$$

The tree-level value is given by (no summation over $\mu$ )

$$
\mathcal{V}_{\mu}^{(0)}=\left\langle V_{\mu}^{(0)}\right\rangle=4 i N_{c} N_{f}\left\{F_{\mu \mu}^{(4)}+a F_{\mu}^{(5)}\right\},
$$

and its derivative with respect to the bare mass is

$$
\frac{\partial \mathcal{V}_{\mu}^{(0)}}{\partial m_{0}}=4 i N_{c} N_{f}\left\{\frac{a^{2} F_{\mu}^{(5)}+\sum_{\sigma} a F_{\mu \sigma}^{(4)}}{\left(a m_{0}+4\right)}-2\left(F_{\mu \mu}^{(6)}+a F_{\mu}^{(7)}\right)\right\} .
$$

The 1-loop contribution is

$$
\mathcal{V}_{\mu}^{(1)}=i\left(N_{c}^{2}-1\right) N_{f}\left\{\mathcal{V}_{\mu}^{1}+\mathcal{V}_{\mu}^{2}+\mathcal{V}_{\mu}^{3}\right\}
$$

where

$$
\begin{aligned}
\left\langle V_{\mu}^{(2)}\right\rangle_{\text {con }} & =i\left(N_{c}^{2}-1\right) N_{f} \mathcal{V}_{\mu}^{1}, \\
\left\langle V_{\mu}^{(1)}\left(-S^{F, 1}\right)\right\rangle_{\text {con }} & =i\left(N_{c}^{2}-1\right) N_{f} \mathcal{V}_{\mu}^{2}, \\
\left\langle V_{\mu}^{(0)}\left[\frac{1}{2}\left(S^{F, 1}\right)^{2}-S^{F, 2}\right]\right\rangle_{\mathrm{con}} & =i\left(N_{c}^{2}-1\right) N_{f} \mathcal{V}_{\mu}^{3} .
\end{aligned}
$$

The expressions of $\mathcal{V}_{\mu}^{1}, \mathcal{V}_{\mu}^{2}$, and $\mathcal{V}_{\mu}^{3}$ in terms of the integrals defined in appendix $\mathrm{J}$ are

$$
\begin{gathered}
\mathcal{V}_{\mu}^{1}=-a^{2} B^{(0)}\left\{F_{\mu \mu}^{(4)}+a F_{\mu}^{(5)}\right\}, \\
\mathcal{V}_{\mu}^{2}=-2 a \int_{q_{\boldsymbol{\xi}} ; p_{\boldsymbol{\xi}, \theta} ; k_{\boldsymbol{\xi}, \theta}} \frac{\bar{\delta}(p-q-k)}{D_{G}(q) D_{F}(p) D_{F}(k)} \\
\times\left\{a \bar{r}_{\mu}\left[m_{0}(k) m_{0}(p)-\bar{k}_{\mu} \bar{p}_{\mu}\right]+\mathrm{c}_{\mu}(r)\left[m_{0}(p) \bar{k}_{\mu}+m_{0}(k) \bar{p}_{\mu}\right]\right\},
\end{gathered}
$$

and

$$
\begin{aligned}
\mathcal{V}_{\mu}^{3}= & B^{(0)}\left\{a^{2} F_{\mu \mu}^{(4)}+2 a^{3} F_{\mu}^{(5)}+a^{2} \sum_{\sigma} F_{\mu \sigma}^{(4)}-2\left(a m_{0}+4\right)\left[a F_{\mu \mu}^{(6)}+a^{2} F_{\mu}^{(7)}\right]\right\} \\
- & -2 \int_{q_{\boldsymbol{\xi}} ; p_{\xi, \theta} ; k_{\xi, \theta}} \frac{\bar{\delta}(p-q-k)}{D_{G}(q) D_{F}^{2}(k) D_{F}(p)}\left\{2 \overline { k } _ { \mu } [ \mathrm { c } _ { \mu } ( k ) + a m _ { 0 } ( k ) ] \left\{m_{0}(p) m_{0}(k) \sum_{\sigma} \mathrm{c}_{\sigma}(r)\right.\right. \\
& \left.\quad-a \sum_{\sigma}\left\{\bar{r}_{\sigma}\left[m_{0}(k) \bar{p}_{\sigma}+m_{0}(p) \bar{k}_{\sigma}\right]\right\}+\sum_{\sigma}\left\{\bar{p}_{\sigma} \bar{k}_{\sigma}\left[3-\mathrm{c}_{\sigma}(r)\right]\right\}\right\} \\
+ & \left.D_{F}(k)\left\{a m_{0}(p) \bar{r}_{\mu} \mathrm{c}_{\mu}(k)+\bar{p}_{\mu} \mathrm{c}_{\mu}(k)\left[\mathrm{c}_{\mu}(r)-3\right]-a \bar{k}_{\mu}\left[m_{0}(p) \sum_{\sigma} \mathrm{c}_{\sigma}(r)-a \sum_{\sigma} \bar{r}_{\sigma} \bar{p}_{\sigma}\right]\right\}\right\}
\end{aligned}
$$

where we have defined $r=p+k$. 


\section{G.1 $\mathrm{O}(a)$-improved action}

The Sheikholeslami-Wohlert terms leads to 3 additional terms to the 1-loop coefficient

$$
\mathcal{V}_{\mu}^{(1)} \longrightarrow \mathcal{V}_{\mu}^{(1)}+i\left(N_{c}^{2}-1\right) N_{f}\left\{\mathcal{V}_{\mu}^{4}+\mathcal{V}_{\mu}^{5}+\mathcal{V}_{\mu}^{6}\right\}
$$

where

$$
\begin{aligned}
\left\langle V_{\mu}^{(0)} S^{S W, 1} S^{F, 1}\right\rangle_{\mathrm{con}} & =i\left(N_{c}^{2}-1\right) N_{f} \mathcal{V}_{\mu}^{4}, \\
\left\langle V_{\mu}^{(0)} \frac{\left(S^{S W, 1}\right)^{2}}{2}\right\rangle_{\mathrm{con}} & =i\left(N_{c}^{2}-1\right) N_{f} \mathcal{V}_{\mu}^{5}, \\
\left\langle V_{\mu}^{(1)}\left(-S^{S W, 1}\right)\right\rangle_{\mathrm{con}} & =i\left(N_{c}^{2}-1\right) N_{f} \mathcal{V}_{\mu}^{6} .
\end{aligned}
$$

The expressions of $\mathcal{V}_{\mu}^{4}, \mathcal{V}_{\mu}^{5}$, and $\mathcal{V}_{\mu}^{6}$ in terms of the integrals defined in appendix $\mathrm{J}$ are

$$
\begin{aligned}
& \mathcal{V}_{\mu}^{4}=a c_{\mathrm{sw}} \int_{q_{\boldsymbol{\xi}} ; p_{\boldsymbol{\xi}, \theta} ; k_{\boldsymbol{\xi}, \theta}} \frac{\bar{\delta}(p-q-k)}{D_{G}(q) D_{F}^{2}(k) D_{F}(p)}\left\{2 \bar{k}_{\mu}\left[\mathrm{c}_{\mu}(k)+a m_{0}(k)\right]\right. \\
& \times\left\{a \sum_{\sigma \rho}\left\{\bar{q}_{\sigma}\left(\bar{k}_{\sigma} \bar{p}_{\rho}-\bar{p}_{\sigma} \bar{k}_{\rho}\right)\left(\bar{p}_{\rho}+\bar{k}_{\rho}\right)\right\}\right. \\
& \left.+\sum_{\sigma}\left\{\bar{q}_{\sigma}\left[m_{0}(k) \bar{p}_{\sigma}-m_{0}(p) \bar{k}_{\sigma}\right] \sum_{\rho \neq \sigma}\left[\mathrm{c}_{\rho}(p)+\mathrm{c}_{\rho}(k)\right]\right\}\right\} \\
& +D_{F}(k)\left\{\mathrm { c } _ { \mu } ( k ) \left\{a\left(\bar{p}_{\mu}+\bar{k}_{\mu}\right) \sum_{\sigma} \bar{q}_{\sigma} \bar{p}_{\sigma}+\bar{q}_{\mu}\left[m_{0}(p) \sum_{\sigma \neq \mu}\left(\mathrm{c}_{\sigma}(p)+\mathrm{c}_{\sigma}(k)\right)\right.\right.\right. \\
& \left.\left.\left.-a \sum_{\sigma} \bar{p}_{\sigma}\left(\bar{p}_{\sigma}+\bar{k}_{\sigma}\right)\right]\right\}-a \bar{k}_{\mu} \sum_{\sigma}\left[\bar{q}_{\sigma} \bar{p}_{\sigma} \sum_{\rho \neq \sigma}\left(\mathrm{c}_{\rho}(p)+\mathrm{c}_{\rho}(k)\right)\right]\right\}, \\
& \mathcal{V}_{\mu}^{5}=\frac{a^{2} c_{\mathrm{SW}}^{2}}{4} \int_{q_{\boldsymbol{\xi}} ; p_{\boldsymbol{\xi}, \theta} ; k_{\boldsymbol{\xi}, \theta}} \frac{\bar{\delta}(p-q-k)}{D_{G}(q) D_{F}^{2}(k) D_{F}(p)}\left\{2 \bar{k}_{\mu}\left[\mathrm{c}_{\mu}(k)+a m_{0}(k)\right]\right. \\
& \times\left\{2 \sum_{\sigma} \bar{q}_{\sigma}^{2} \sum_{\rho} \bar{p}_{\rho} \bar{k}_{\rho}\left(1+\mathrm{c}_{\rho}(q)\right)+2 \sum_{\sigma} \bar{k}_{\sigma} \bar{q}_{\sigma} \sum_{\rho} \bar{q}_{\rho} \bar{p}_{\rho}\left(2-\mathrm{c}_{\sigma}(q)+\sum_{\lambda \neq \rho} \mathrm{c}_{\lambda}(q)\right)\right. \\
& \left.-\left[\sum_{\sigma} \bar{p}_{\sigma} \bar{k}_{\sigma}-m_{0}(k) m_{0}(p)\right]\left[\sum_{\rho} \bar{q}_{\rho}^{2}\left(3+\sum_{\lambda \neq \rho} c_{\lambda}(q)\right)\right]\right\} \\
& +D_{F}(k)\left\{\mathrm { c } _ { \mu } ( k ) \left\{\bar{p}_{\mu} \sum_{\sigma}\left[\bar{q}_{\sigma}^{2}\left(1-2 \mathrm{c}_{\mu}(q)+\sum_{\rho \neq \sigma} \mathrm{c}_{\rho}(q)\right)\right]-2 \bar{q}_{\mu} \sum_{\sigma}\left[\bar{p}_{\sigma} \bar{q}_{\sigma}\right.\right.\right. \\
& \left.\left.\left.\left.\times\left(2-\mathrm{c}_{\mu}(q)+\sum_{\rho \neq \sigma} \mathrm{c}_{\rho}(q)\right)\right]\right\}-\operatorname{am}_{0}(p) \bar{k}_{\mu} \sum_{\sigma}\left\{\bar{q}_{\sigma}^{2}\left(3+\sum_{\rho \neq \sigma} \mathrm{c}_{\rho}(q)\right)\right\}\right\}\right\},
\end{aligned}
$$


and

$$
\begin{aligned}
& \mathcal{V}_{\mu}^{6}= \frac{a^{2} c_{\mathrm{Sw}}}{2} \int_{q_{\boldsymbol{\xi}} ; p_{\boldsymbol{\xi}, \theta} ; k_{\boldsymbol{\xi}, \theta}} \frac{\bar{\delta}(p-q-k)}{D_{G}(q) D_{F}(p) D_{F}(k)}\left\{a\left[\bar{p}_{\mu}+\bar{k}_{\mu}\right]\right. \\
&\left.\times\left[m_{0}(k) \sum_{\sigma \neq \mu} \bar{p}_{\sigma} \bar{q}_{\sigma}-m_{0}(p) \sum_{\sigma \neq \mu} \bar{k}_{\sigma} \bar{q}_{\sigma}\right]-\left[\mathrm{c}_{\mu}(p)+\mathrm{c}_{\mu}(k)\right] \sum_{\sigma}\left[\bar{q}_{\sigma}\left(\bar{p}_{\mu} \bar{k}_{\sigma}-\bar{k}_{\mu} \bar{p}_{\sigma}\right)\right]\right\} .
\end{aligned}
$$

\section{H Expectation values of sextet components of $T_{\mu \nu}$}

In this appendix we report the coefficients of the 1-loop perturbative expansion of the expectation values of the sextet components of the energy-momentum tensor defined in eqs. (5.4)-(5.6). By expanding the field strength $F_{\mu \nu}$ to order $g_{0}^{2}$

$$
F_{\mu \nu}^{a}(x)=F_{\mu \nu}^{a(0)}(x)+g_{0} F_{\mu \nu}^{a(1)}(x)+g_{0}^{2} F_{\mu \nu}^{a(2)}(x)+\mathcal{O}\left(g_{0}^{3}\right),
$$

the tree-level value of the gluonic part is given by

$$
\mathcal{T}_{\mu \nu}^{G\{6\}(0)}=\frac{1}{N_{c}^{2}-1}\left(1-\delta_{\mu \nu}\right) \sum_{\alpha \neq \mu, \nu}\left\langle F_{\mu \alpha}^{a(0)} F_{\nu \alpha}^{a(0)}\right\rangle=\left(1-\delta_{\mu \nu}\right) \sum_{\alpha \neq \mu, \nu} B_{\mu \nu \alpha}^{(2)} .
$$

The 1-loop contributions to the gluonic component are

$$
\begin{aligned}
& \mathcal{T}_{\mu \nu}^{G\{6\}\left(1, N_{c}\right)}=\left(1-\delta_{\mu \nu}\right)\left\{\mathcal{T}_{\mu \nu}^{G 1}+\mathcal{T}_{\mu \nu}^{G 2}+\mathcal{T}_{\mu \nu}^{G 3}\right\} \\
& \mathcal{T}_{\mu \nu}^{G\{6\}\left(1, \frac{1}{N_{c}}\right)}=\left(1-\delta_{\mu \nu}\right)\left\{\mathcal{T}_{\mu \nu}^{G 4}+\mathcal{T}_{\mu \nu}^{G 5}\right\} \\
& \mathcal{T}_{\mu \nu}^{G\{6\}\left(1, N_{f}\right)}=\left(1-\delta_{\mu \nu}\right)\left\{\mathcal{T}_{\mu \nu}^{G 6}+\mathcal{T}_{\mu \nu}^{G 7}\right\}
\end{aligned}
$$

where

$$
\begin{aligned}
& \sum_{\alpha \neq \mu, \nu}\left\langle\left[F_{\mu \alpha}^{a(0)} F_{\nu \alpha}^{a(2)}+F_{\mu \alpha}^{a(2)} F_{\nu \alpha}^{a(0)}+F_{\mu \alpha}^{a(1)} F_{\nu \alpha}^{a(1)}\right]\right\rangle_{\mathrm{con}}=\left(N_{c}^{2}-1\right)\left(N_{c} \mathcal{T}_{\mu \nu}^{G 1}+\frac{1}{N_{c}} \mathcal{T}_{\mu \nu}^{G 4}\right), \\
& \sum_{\alpha \neq \mu, \nu}\left\langle\left[F_{\mu \alpha}^{a(0)} F_{\nu \alpha}^{a(1)}+F_{\mu \alpha}^{a(1)} F_{\nu \alpha}^{a(0)}\right]\left(-S^{G, 1}\right)\right\rangle_{\mathrm{con}}=\left(N_{c}^{2}-1\right) N_{c} \mathcal{T}_{\mu \nu}^{G 2}, \\
& \sum_{\alpha \neq \mu, \nu}\left\langle\left[F_{\mu \alpha}^{a(0)} F_{\nu \alpha}^{a(0)}\right]\left(\frac{\left(S^{G, 1}\right)^{2}+\left(S^{F P, 1}\right)^{2}}{2}-S^{G, 2}-S^{F P, 2}-S^{M, 2}\right)\right\rangle_{\mathrm{con}}=\left(N_{c}^{2}-1\right)\left(N_{c} \mathcal{T}_{\mu \nu}^{G 3}+\frac{1}{N_{c}} \mathcal{T}_{\mu \nu}^{G 5}\right), \\
& \sum_{\alpha \neq \mu, \nu}\left\langle\left[F_{\mu \alpha}^{a(0)} F_{\nu \alpha}^{a(0)}\right]\left(-S^{F, 2}\right)\right\rangle_{\mathrm{con}}=\left(N_{c}^{2}-1\right) N_{f} \mathcal{T}_{\mu \nu}^{G 6}, \\
& \sum_{\alpha \neq \mu, \nu}\left\langle\left[F_{\mu \alpha}^{a(0)} F_{\nu \alpha}^{a(0)}\right] \frac{\left(S^{F, 1}\right)^{2}}{2}\right\rangle_{\mathrm{con}}=\left(N_{c}^{2}-1\right) N_{f} \mathcal{T}_{\mu \nu}^{G 7} .
\end{aligned}
$$

The expressions of $\mathcal{T}_{\mu \nu}^{G 1}, \ldots, \mathcal{T}_{\mu \nu}^{G 7}$ in terms of the integrals defined in appendix $\mathrm{J}$ are

$$
\begin{aligned}
\mathcal{T}_{\mu \nu}^{G 1}= & \frac{a^{2}}{8} \sum_{\alpha \neq \mu, \nu}\left\{2\left[B_{\mu}^{(3)}+B_{\nu}^{(3)}-B_{\alpha}^{(3)}-\frac{22}{3} B^{(0)}\right] B_{\mu \nu \alpha}^{(2)}-a^{2} B_{\mu \alpha}^{(4)} B_{\nu \alpha}^{(4)}+B_{\mu \nu}^{(4)}\left[B^{(0)}+B_{\alpha}^{(3)}\right]\right. \\
& +\delta_{\mu \nu}\left[2\left[B^{(0)}+B_{\alpha}^{(3)}\right]\left[B^{(0)}+B_{\mu}^{(3)}\right]-3 B_{\mu \alpha}^{(4)} B_{\mu \alpha}^{(4)}-4\left[B_{\mu}^{(3)}+B_{\mu \alpha}^{(6)}+B_{\alpha \alpha}^{(4)}-2 B_{\alpha \alpha \mu}^{(2)}\right] B_{\mu \mu \alpha}^{(2)}\right.
\end{aligned}
$$




$$
\begin{aligned}
& \left.\left.+8 B_{\mu \alpha \alpha}^{(2)} B_{\alpha \mu \mu}^{(2)}+\left[B^{(0)}+B_{\mu}^{(3)}+2 B_{\mu \mu}^{(4)}\right] B_{\alpha \alpha}^{(4)}-4\left[\frac{1}{2} B_{\mu}^{(3)}+B_{\mu \alpha}^{(6)}+B_{\mu \mu}^{(4)}+\frac{11}{3} B^{(0)}\right] B_{\alpha \alpha \mu}^{(2)}\right]\right\} \\
& \mathcal{T}_{\mu \nu}^{G 2}=\frac{1}{2} \int_{p_{\boldsymbol{\xi}} ; q_{\boldsymbol{\xi}} ; k_{\xi}} \frac{\bar{\delta}(p+q+k)}{D_{G}(p) D_{G}(q) D_{G}(k)} \sum_{\alpha \neq \mu, \nu}\left\{a^{2} \bar{p}_{\mu} \bar{p}_{\nu} \bar{q}_{\alpha}\left[2 \bar{q}_{\alpha}-\bar{k}_{\alpha}-\bar{p}_{\alpha}\right]\right. \\
& -a^{2} \bar{p}_{\mu} \bar{q}_{\nu}\left(\bar{k}_{\alpha}-\bar{p}_{\alpha}\right)\left(\bar{k}_{\alpha}-\bar{q}_{\alpha}\right)-\frac{1}{2}\left(1+\mathrm{c}_{\alpha}(p)\right)\left(1+\mathrm{c}_{\alpha}(k)\right)\left[\bar{p}_{\mu}(\overline{p-q})_{\nu}+\bar{p}_{\nu}(\overline{p-q})_{\mu}\right] \\
& \left.+\delta_{\mu \nu}\left[a^{2} \bar{p}_{\mu} \bar{q}_{\mu} \bar{p}_{\alpha}\left(\bar{q}_{\alpha}-\bar{p}_{\alpha}\right)-\left(1+\mathrm{c}_{\mu}(p)\right)\left(1+\mathrm{c}_{\mu}(k)\right) \bar{p}_{\alpha}(\overline{p-q})_{\alpha}\right]\right\}, \\
& \mathcal{T}_{\mu \nu}^{G 3}=\sum_{\alpha \neq \mu, \nu}\left[\frac{5}{6} B^{(0)}\left(a^{2} B_{\mu \nu \alpha}^{(2)}-3 B_{\mu \nu \alpha}^{(1)}\right)+\frac{1}{4 a^{2}} B_{\mu \nu \alpha}^{(1)}\right. \\
& \left.+\frac{1}{2} B_{\alpha}^{(3)}\left(B_{\mu \nu \alpha \alpha}^{(5)}-6 B_{\mu \nu \alpha}^{(1)}\right)+\frac{1}{2} \sum_{\sigma} B_{\sigma}^{(3)} B_{\mu \nu \alpha \sigma}^{(5)}\right] \\
& +\frac{1}{8} \int_{p_{\xi} ; q_{\xi} ; k_{\xi}} \frac{\bar{\delta}(p+q+k)}{D_{G}(p)^{2} D_{G}(q) D_{G}(k)} \sum_{\alpha \neq \mu, \nu}\left\{[ 8 - a ^ { 2 } D _ { G } ( p ) ] \left[\bar{p}_{\mu} \bar{p}_{\nu}\left[\bar{q}_{\alpha}-\bar{k}_{\alpha}\right]^{2}\right.\right. \\
& \left.-\bar{p}_{\mu} \bar{p}_{\alpha}\left[\bar{q}_{\alpha}-\bar{k}_{\alpha}\right]\left[\bar{q}_{\nu}-\bar{k}_{\nu}\right]-\bar{p}_{\nu} \bar{p}_{\alpha}\left[\bar{q}_{\mu}-\bar{k}_{\mu}\right]\left[\bar{q}_{\alpha}-\bar{k}_{\alpha}\right]+\bar{p}_{\alpha}^{2}\left[\bar{q}_{\mu}-\bar{k}_{\mu}\right]\left[\bar{q}_{\nu}-\bar{k}_{\nu}\right]\right] \\
& -2 \bar{p}_{\mu} \bar{p}_{\nu}\left[a^{4} \bar{p}_{\alpha}^{2}\left(\hat{q}_{\mu}^{2}-\hat{q}_{\alpha}^{2}\right)\left(\hat{k}_{\nu}^{2}-\hat{k}_{\alpha}^{2}\right)-\left(1+\mathrm{c}_{\alpha}(p)\right)\left(1+\mathrm{c}_{\alpha}(q)\right) \sum_{\sigma}(\widehat{p-k})_{\sigma}^{2}\right] \\
& \left.+2 \delta_{\mu \nu} \bar{p}_{\alpha}^{2}\left(1+\mathrm{c}_{\mu}(p)\right)\left(1+\mathrm{c}_{\mu}(q)\right) \sum_{\sigma}(\widehat{p-k})_{\sigma}^{2}\right\}+\delta_{\mu \nu} \sum_{\alpha \neq \mu}\left[\frac{5}{6} B^{(0)}\left(a^{2} B_{\alpha \alpha \mu}^{(2)}-3 B_{\alpha \alpha \mu}^{(1)}\right)\right. \\
& \left.+\frac{1}{4 a^{2}} B_{\alpha \alpha \mu}^{(1)}+\frac{1}{2} B_{\mu}^{(3)}\left(B_{\alpha \alpha \mu \mu}^{(5)}-6 B_{\alpha \alpha \mu}^{(1)}\right)+\frac{1}{2} \sum_{\sigma} B_{\sigma}^{(3)} B_{\alpha \alpha \mu \sigma}^{(5)}\right], \\
& \mathcal{T}_{\mu \nu}^{G 4}=a^{2} \sum_{\alpha \neq \mu, \nu}\left\{2 B^{(0)}-\frac{1}{2}\left[B_{\mu}^{(3)}+B_{\nu}^{(3)}\right]-B_{\alpha}^{(3)}\right\}\left[B_{\mu \nu \alpha}^{(2)}+\delta_{\mu \nu} B_{\alpha \alpha \mu}^{(2)}\right], \\
& \mathcal{T}_{\mu \nu}^{G 5}=-\frac{1}{2} \sum_{\alpha \neq \mu, \nu}\left\{a^{2} B_{\mu \nu \alpha}^{(2)}\left(2 B^{(0)}-B_{\alpha}^{(3)}\right)+\left(\frac{1}{a^{2}}-8 B^{(0)}\right) B_{\mu \nu \alpha}^{(1)}+2 \sum_{\sigma} B_{\sigma}^{(3)} B_{\mu \nu \alpha \sigma}^{(5)}\right. \\
& \left.+\delta_{\mu \nu}\left[a^{2} B_{\alpha \alpha \mu}^{(2)}\left(2 B^{(0)}-B_{\mu}^{(3)}\right)+\left(\frac{1}{a^{2}}-8 B^{(0)}\right) B_{\alpha \alpha \mu}^{(1)}+2 \sum_{\sigma} B_{\sigma}^{(3)} B_{\alpha \alpha \mu \sigma}^{(5)}\right]\right\}, \\
& \mathcal{T}_{\mu \nu}^{G 6}=-2 a \sum_{\alpha \neq \mu, \nu}\left\{\left(a F_{\alpha}^{(2)}-F_{\alpha}^{(3)}\right) B_{\mu \nu \alpha}^{(1)}+\delta_{\mu \nu}\left(a F_{\mu}^{(2)}-F_{\mu}^{(3)}\right) B_{\alpha \alpha \mu}^{(1)}\right\}, \\
& \mathcal{T}_{\mu \nu}^{G 7}=-2 \sum_{\alpha \neq \mu, \nu}\left(h_{\mu \nu \alpha \alpha}^{(1)}-h_{\mu \alpha \alpha \nu}^{(1)}-h_{\alpha \nu \mu \alpha}^{(1)}+h_{\alpha \alpha \mu \nu}^{(1)}\right),
\end{aligned}
$$


where

$$
\begin{aligned}
h_{\rho \sigma \mu \nu}^{(1)}= & \frac{1}{4} \int_{q_{\boldsymbol{\xi}} ; p_{\boldsymbol{\xi}, \theta} ; k_{\boldsymbol{\xi}, \theta}} \frac{\bar{\delta}(p-q-k) \bar{q}_{\rho} \bar{q}_{\sigma}}{D_{G}(q)^{2} D_{F}(p) D_{F}(k)} \\
\times & \left\{a^{2}\left[\bar{p}_{\mu}+\bar{k}_{\mu}\right]\left[\bar{p}_{\nu}+\bar{k}_{\nu}\right]\left[m_{0}(p) m_{0}(k)-\sum_{\sigma} \bar{p}_{\sigma} \bar{k}_{\sigma}\right]\right. \\
& +\left[\mathrm{c}_{\mu}(p)+\mathrm{c}_{\mu}(k)\right]\left[\mathrm{c}_{\nu}(p)+\mathrm{c}_{\nu}(k)\right]\left[\bar{k}_{\mu} \bar{p}_{\nu}+\bar{p}_{\mu} \bar{k}_{\nu}\right] \\
& +a\left[\mathrm{c}_{\nu}(p)+\mathrm{c}_{\nu}(k)\right]\left[\bar{p}_{\mu}+\bar{k}_{\mu}\right]\left[m_{0}(p) \bar{k}_{\nu}+m_{0}(k) \bar{p}_{\nu}\right] \\
& +a\left[\mathrm{c}_{\mu}(p)+\mathrm{c}_{\mu}(k)\right]\left[\bar{p}_{\nu}+\bar{k}_{\nu}\right]\left[m_{0}(p) \bar{k}_{\mu}+m_{0}(k) \bar{p}_{\mu}\right] \\
& \left.-\delta_{\mu \nu}\left[\mathrm{c}_{\mu}(p)+\mathrm{c}_{\mu}(k)\right]^{2}\left[m_{0}(p) m_{0}(k)+\sum_{\sigma} \bar{p}_{\sigma} \bar{k}_{\sigma}\right]\right\} .
\end{aligned}
$$

By expanding the fermion part of the energy-momentum tensor in eq. (3.4) to order $g_{0}^{2}$

$$
T_{\mu \nu}^{F}(x)=T_{\mu \nu}^{F(0)}(x)+g_{0} T_{\mu \nu}^{F(1)}(x)+g_{0}^{2} T_{\mu \nu}^{F(2)}(x)+\mathcal{O}\left(g_{0}^{3}\right),
$$

the tree-level value of the expectation value of $T_{\mu \nu}^{F,\{6\}}$ is

$$
\mathcal{T}_{\mu \nu}^{F\{6\}(0)}=\frac{1}{N_{c} N_{f}}\left(1-\delta_{\mu \nu}\right)\left\langle T_{\mu \nu}^{F(0)}\right\rangle=-4\left(1-\delta_{\mu \nu}\right) F_{\mu \nu}^{(0)}
$$

and its derivative with respect to the bare mass is

$$
\frac{\partial \mathcal{T}_{\mu \nu}^{F\{6\}(0)}}{\partial m_{0}}=8\left(1-\delta_{\mu \nu}\right) F_{\mu \nu}^{(1)}
$$

The 1-loop fermion contribution is

$$
\mathcal{T}_{\mu \nu}^{F\{6\}\left(1, N_{f}\right)}=\left(1-\delta_{\mu \nu}\right)\left\{\mathcal{T}_{\mu \nu}^{F 1}+\mathcal{T}_{\mu \nu}^{F 2}+\mathcal{T}_{\mu \nu}^{F 3}\right\}
$$

where

$$
\begin{aligned}
\left\langle T_{\mu \nu}^{F(2)}\right\rangle_{\mathrm{con}} & =\left(N_{c}^{2}-1\right) N_{f} \mathcal{T}_{\mu \nu}^{F 1}, \\
\left\langle T_{\mu \nu}^{F(1)}\left(-S^{F, 1}\right)\right\rangle_{\mathrm{con}} & =\left(N_{c}^{2}-1\right) N_{f} \mathcal{T}_{\mu \nu}^{F 2}, \\
\left\langle T_{\mu \nu}^{F(0)}\left(\frac{\left(S^{F, 1}\right)^{2}}{2}-S^{F, 2}\right)\right\rangle_{\text {con }} & =\left(N_{c}^{2}-1\right) N_{f} \mathcal{T}_{\mu \nu}^{F 3} .
\end{aligned}
$$


The expressions of $\mathcal{T}_{\mu \nu}^{F 1}, \mathcal{T}_{\mu \nu}^{F 2}$, and $\mathcal{T}_{\mu \nu}^{F 3}$ in terms of integrals defined in appendix $\mathrm{J}$ are

$$
\begin{aligned}
\mathcal{T}_{\mu \nu}^{F 1}= & a^{2} B^{(0)} F_{\mu \nu}^{(0)}, \\
\mathcal{T}_{\mu \nu}^{F 2}= & \frac{1}{2} \int_{q_{\boldsymbol{\xi}} ; p_{\boldsymbol{\xi}, \theta} ; k_{\boldsymbol{\xi}, \theta}} \frac{\bar{\delta}(p-q-k)}{D_{G}(q) D_{F}(p) D_{F}(k)}\left\{a \bar{r}_{\nu}\left[m_{0}(p) \bar{k}_{\mu}+m_{0}(k) \bar{p}_{\mu}\right]\right. \\
+ & a \bar{r}_{\mu}\left[m_{0}(p) \bar{k}_{\nu}+m_{0}(k) \bar{p}_{\nu}\right]+\left[2+\mathrm{c}_{\mu}(r)+\mathrm{c}_{\nu}(r)\right]\left[\bar{k}_{\mu} \bar{p}_{\nu}+\bar{k}_{\nu} \bar{p}_{\mu}\right] \\
& \left.-2 \delta_{\mu \nu}\left[1+\mathrm{c}_{\mu}(r)\right]\left[m_{0}(k) m_{0}(p)+\sum_{\sigma} \bar{k}_{\sigma} \bar{p}_{\sigma}\right]\right\} \\
\mathcal{T}_{\mu \nu}^{F 3}= & \left\{\left[2 a\left(a m_{0}+4\right) B^{(0)} F_{\mu \nu}^{(1)}-a^{2} B^{(0)} F_{\mu \nu}^{(0)}\right]+\int_{q_{\boldsymbol{\xi}} ; p_{\boldsymbol{\xi}, \theta} ; k_{\boldsymbol{\xi}, \theta}} \frac{D_{G}(q) D_{F}^{2}(k) D_{F}(p)}{}\right. \\
& \times\left\{4 \overline { k } _ { \mu } \overline { k } _ { \nu } \left[m_{0}(p)\left[m_{0}(k) \sum_{\sigma} c_{\sigma}(r)-a \sum_{\sigma} \bar{k}_{\sigma} \bar{r}_{\sigma}\right]\right.\right. \\
& \left.\quad-a m_{0}(k) \sum_{\sigma} \bar{p}_{\sigma} \bar{r}_{\sigma}+\sum_{\sigma}\left[3-\mathrm{c}_{\sigma}(r)\right] \bar{p}_{\sigma} \bar{k}_{\sigma}\right] \\
& \left.\left.+D_{F}(k)\left[a m_{0}(p)\left(\bar{r}_{\mu} \bar{k}_{\nu}+\bar{k}_{\mu} \bar{r}_{\nu}\right)+\bar{p}_{\mu} \bar{k}_{\nu}\left(\mathrm{c}_{\mu}(r)-3\right)+\bar{p}_{\nu} \bar{k}_{\mu}\left(\mathrm{c}_{\nu}(r)-3\right)\right]\right\}\right\},
\end{aligned}
$$

where $r=p+k$.

\section{H.1 $\mathrm{O}(a)$-improved action}

The 1-loop gluonic contribution to the off-diagonal components of the energy-momentum due to the improvement term is

$$
\mathcal{T}_{\mu \nu}^{G\{6\}\left(1, N_{f}\right)} \longrightarrow \mathcal{T}_{\mu \nu}^{G\{6\}\left(1, N_{f}\right)}+\left(1-\delta_{\mu \nu}\right)\left\{\mathcal{T}_{\mu \nu}^{G 8}+\mathcal{T}_{\mu \nu}^{G 9}\right\}
$$

where

$$
\begin{aligned}
& \sum_{\alpha \neq \mu, \nu}\left\langle\left[F_{\mu \alpha}^{a(0)} F_{\nu \alpha}^{a(0)}\right]\left(S^{S W, 1} S^{F, 1}\right)\right\rangle_{\mathrm{con}}=\left(N_{c}^{2}-1\right) N_{f} \mathcal{T}_{\mu \nu}^{G 8}, \\
& \sum_{\alpha \neq \mu, \nu}\left\langle\left[F_{\mu \alpha}^{a(0)} F_{\nu \alpha}^{a(0)}\right] \frac{\left(S^{S W, 1}\right)^{2}}{2}\right\rangle_{\mathrm{con}}=\left(N_{c}^{2}-1\right) N_{f} \mathcal{T}_{\mu \nu}^{G 9} .
\end{aligned}
$$

The expressions of $\mathcal{T}_{\mu \nu}^{G 8}$ and $\mathcal{T}_{\mu \nu}^{G 9}$ in terms of the integrals defined in appendix $\mathrm{J}$ are

$$
\begin{aligned}
& \mathcal{T}_{\mu \nu}^{G 8}=-a c_{\mathrm{sw}} \sum_{\alpha \neq \mu, \nu}\left(h_{\mu \nu \alpha \alpha}^{(2)}-h_{\mu \alpha \alpha \nu}^{(2)}-h_{\alpha \nu \mu \alpha}^{(2)}+h_{\alpha \alpha \mu \nu}^{(2)}\right), \\
& \mathcal{T}_{\mu \nu}^{G 9}=-\frac{a^{2} c_{\mathrm{sw}}^{2}}{4} \sum_{\alpha \neq \mu, \nu}\left(h_{\mu \nu \alpha \alpha}^{(3)}-h_{\mu \alpha \alpha \nu}^{(3)}-h_{\alpha \nu \mu \alpha}^{(3)}+h_{\alpha \alpha \mu \nu}^{(3)}\right),
\end{aligned}
$$


where

$$
\begin{aligned}
& h_{\rho \sigma \mu \nu}^{(2)}=\frac{1}{4} \int_{q_{\boldsymbol{\xi}} ; p_{\xi, \theta} ; k_{\xi, \theta}} \frac{\bar{\delta}(p-q-k) \bar{q}_{\rho} \bar{q}_{\sigma}}{D_{G}(q)^{2} D_{F}(p) D_{F}(k)}\left\{\left\{\left(1+\mathrm{c}_{\nu}(q)\right)\right.\right. \\
& \times\left[a\left(\bar{p}_{\mu}+\bar{k}_{\mu}\right)\left(\bar{p}_{\nu} \sum_{\sigma} \bar{q}_{\sigma} \bar{k}_{\sigma}-\bar{k}_{\nu} \sum_{\sigma} \bar{q}_{\sigma} \bar{p}_{\sigma}\right)+\left(\mathrm{c}_{\mu}(p)+\mathrm{c}_{\mu}(k)\right) \bar{q}_{\mu}\left(m_{0}(p) \bar{k}_{\nu}-m_{0}(k) \bar{p}_{\nu}\right)\right] \\
& \left.\quad+(\mu \leftrightarrow \nu)\}+2 \delta_{\mu \nu}\left(1+\mathrm{c}_{\mu}(q)\right)\left(\mathrm{c}_{\mu}(p)+\mathrm{c}_{\mu}(k)\right)\left[m_{0}(k) \sum_{\sigma} \bar{q}_{\sigma} \bar{p}_{\sigma}-m_{0}(p) \sum_{\sigma} \bar{q}_{\sigma} \bar{k}_{\sigma}\right]\right\},
\end{aligned}
$$

and

$$
\begin{aligned}
& h_{\rho \sigma \mu \nu}^{(3)}=\frac{1}{2} \int_{q_{\boldsymbol{\xi}} ; p_{\boldsymbol{\xi}, \theta} ; k_{\boldsymbol{\xi}, \theta}} \frac{\bar{\delta}(p-q-k) \bar{q}_{\rho} \bar{q}_{\sigma}}{D_{G}(q)^{2} D_{F}(p) D_{F}(k)}\left(1+\mathrm{c}_{\mu}(q)\right)\left(1+\mathrm{c}_{\nu}(q)\right)\left\{\bar{q}_{\mu} \bar{q}_{\nu} \sum_{\sigma} \bar{p}_{\sigma} \bar{k}_{\sigma} \quad\right. \text { (H.31) } \\
& \quad+\left(\bar{k}_{\mu} \bar{p}_{\nu}+\bar{k}_{\nu} \bar{p}_{\mu}\right) \sum_{\sigma} \bar{q}_{\sigma}^{2}-m_{0}(k) m_{0}(p) \bar{q}_{\mu} \bar{q}_{\nu}-\left(\bar{k}_{\mu} \bar{q}_{\nu}+\bar{k}_{\nu} \bar{q}_{\mu}\right) \sum_{\sigma} \bar{q}_{\sigma} \bar{p}_{\sigma}-\left(\bar{p}_{\mu} \bar{q}_{\nu}+\bar{p}_{\nu} \bar{q}_{\mu}\right) \\
& \left.\quad \times \sum_{\sigma} \bar{q}_{\sigma} \bar{k}_{\sigma}+\delta_{\mu \nu}\left[\left(m_{0}(k) m_{0}(p)-\sum_{\rho} \bar{k}_{\rho} \bar{p}_{\rho}\right) \sum_{\sigma} \bar{q}_{\sigma}^{2}+2\left(\sum_{\sigma} \bar{q}_{\sigma} \bar{k}_{\sigma}\right)\left(\sum_{\rho} \bar{q}_{\rho} \bar{p}_{\rho}\right)\right]\right\} .
\end{aligned}
$$

The contribution due to the improvement term to the fermionic part of the energymomentum tensor is made up of 3 terms

$$
\mathcal{T}_{\mu \nu}^{F\{6\}\left(1, N_{f}\right)} \longrightarrow \mathcal{T}_{\mu \nu}^{F\{6\}\left(1, N_{f}\right)}+\left(1-\delta_{\mu \nu}\right)\left\{\mathcal{T}_{\mu \nu}^{F 4}+\mathcal{T}_{\mu \nu}^{F 5}+\mathcal{T}_{\mu \nu}^{F 6}\right\}
$$

where

$$
\begin{aligned}
\left\langle T_{\mu \nu}^{F(0)}\left(S^{S W, 1} S^{F, 1}\right)\right\rangle_{\mathrm{con}} & =\left(N_{c}^{2}-1\right) N_{f} \mathcal{T}_{\mu \nu}^{F 4}, \\
\left\langle T_{\mu \nu}^{F(0)} \frac{\left(S^{S W, 1}\right)^{2}}{2}\right\rangle_{\mathrm{con}} & =\left(N_{c}^{2}-1\right) N_{f} \mathcal{T}_{\mu \nu}^{F 5}, \\
\left\langle T_{\mu \nu}^{F(1)}\left(-S^{S W, 1}\right)\right\rangle & =\left(N_{c}^{2}-1\right) N_{f} \mathcal{T}_{\mu \nu}^{F 6} .
\end{aligned}
$$

The expressions of $\mathcal{T}_{\mu \nu}^{F 4}, \mathcal{T}_{\mu \nu}^{F 5}$, and $\mathcal{T}_{\mu \nu}^{F 6}$ in terms of integrals defined in appendix $\mathrm{J}$ are

$$
\begin{aligned}
\mathcal{T}_{\mu \nu}^{F 4}= & -2 a c_{\mathrm{sw}} \int_{q_{\boldsymbol{\xi}} ; p_{\boldsymbol{\xi}, \theta} ; k_{\boldsymbol{\xi}, \theta}} \frac{\bar{\delta}(p-q-k)}{D_{G}(q) D_{F}^{2}(k) D_{F}(p)}\left\{\overline { k } _ { \mu } \overline { k } _ { \nu } \left[a \sum_{\rho \sigma}\left(\left(\bar{p}_{\rho}+\bar{k}_{\rho}\right) \bar{q}_{\sigma}\left(\bar{p}_{\rho} \bar{k}_{\sigma}-\bar{p}_{\sigma} \bar{k}_{\rho}\right)\right)\right.\right. \\
& \left.+\sum_{\substack{\rho \sigma \\
\rho \neq \sigma}} \bar{q}_{\sigma}\left(m_{0}(k) \bar{p}_{\sigma}-m_{0}(p) \bar{k}_{\sigma}\right)\left(\mathrm{c}_{\rho}(p)+\mathrm{c}_{\rho}(k)\right)\right]+\frac{D_{F}(k)}{4}\left\{\overline { k } _ { \nu } \left[a\left(\bar{p}_{\mu}+\bar{k}_{\mu}\right) \sum_{\sigma} \bar{p}_{\sigma} \bar{q}_{\sigma}\right.\right. \\
& \left.\left.\left.-a \bar{q}_{\mu} \sum_{\sigma}\left(\bar{p}_{\sigma}+\bar{k}_{\sigma}\right) \bar{p}_{\sigma}+m_{0}(p) \bar{q}_{\mu} \sum_{\sigma \neq \mu}\left(\mathrm{c}_{\sigma}(p)+\mathrm{c}_{\sigma}(k)\right)\right]+(\mu \leftrightarrow \nu)\right\}\right\}, \\
\mathcal{T}_{\mu \nu}^{F 5}= & a^{2} c_{\mathrm{sw}}^{2} \int_{q_{\boldsymbol{\xi}} ; p_{\boldsymbol{\xi}, \theta} ; k_{\boldsymbol{\xi}, \theta}} \frac{\bar{\delta}(p-q-k)}{D_{G}(q) D_{F}^{2}(k) D_{F}(p)}\left\{\overline { k } _ { \mu } \overline { k } _ { \nu } \left\{\frac{1}{2}\left[\sum_{\sigma} \bar{p}_{\sigma} \bar{k}_{\sigma}-m_{0}(k) m_{0}(p)\right]\right.\right. \\
& \times\left[\sum_{\sigma} \bar{q}_{\sigma}^{2}\left(3+\sum_{\lambda \neq \sigma} \mathrm{c}_{\lambda}(q)\right)\right]-\sum_{\sigma} \bar{p}_{\sigma} \bar{q}_{\sigma} \sum_{\rho} \bar{k}_{\rho} \bar{q}_{\rho}\left(2-\mathrm{c}_{\sigma}(q)+\sum_{\lambda \neq \rho} \mathrm{c}_{\lambda}(q)\right)
\end{aligned}
$$




$$
\begin{aligned}
& \left.-\sum_{\sigma} \bar{q}_{\sigma}^{2} \sum_{\lambda} \bar{k}_{\lambda} \bar{p}_{\lambda}\left(1+\mathrm{c}_{\lambda}(q)\right)\right\}-\frac{D_{F}(k)}{4}\left\{\overline { k } _ { \nu } \left[\frac{1}{2} \bar{p}_{\mu} \sum_{\sigma}\left[\bar{q}_{\sigma}^{2}\left(1-2 \mathrm{c}_{\mu}(q)+\sum_{\lambda \neq \sigma} \mathrm{c}_{\lambda}(q)\right)\right]\right.\right. \\
& \left.\left.\left.-\bar{q}_{\mu}\left[\sum_{\sigma} \bar{p}_{\sigma} \bar{q}_{\sigma}\left(2-\mathrm{c}_{\mu}(q)+\sum_{\lambda \neq \sigma} \mathrm{c}_{\lambda}(q)\right)\right]\right]+(\mu \leftrightarrow \nu)\right\}\right\}
\end{aligned}
$$

and

$$
\begin{array}{r}
\mathcal{T}_{\mu \nu}^{F 6}=\frac{a c_{\mathrm{sw}}}{4} \int_{q_{\boldsymbol{\xi}} ; p_{\boldsymbol{\xi}, \theta} ; k_{\boldsymbol{\xi}, \theta}} \frac{\bar{\delta}(p-q-k)}{D_{G}(q) D_{F}(k) D_{F}(p)}\left\{\left\{\bar{q}_{\mu}\left(\mathrm{c}_{\nu}(p)+\mathrm{c}_{\nu}(k)\right)\left[m_{0}(p) \bar{k}_{\nu}-m_{0}(k) \bar{p}_{\nu}\right]\right.\right. \\
\left.+(\mu \leftrightarrow \nu)\}-2 \delta_{\mu \nu}\left(\mathrm{c}_{\mu}(p)+\mathrm{c}_{\mu}(k)\right) \sum_{\sigma}\left[\bar{q}_{\sigma}\left(m_{0}(p) \bar{k}_{\sigma}-m_{0}(k) \bar{p}_{\sigma}\right)\right]\right\} . \quad(\mathrm{H} .38)
\end{array}
$$

\section{Expectation values of triplet component of $T_{\mu \nu}$}

Here we report the coefficients of the 1-loop perturbative expansion of the expectation values of the triplet components of the energy-momentum tensor defined in eqs. (5.4)(5.6). The tree-level value of the gluonic part is given by (no summation over repeated indices in this appendix unless explicitly indicated)

$$
\mathcal{T}_{\mu \nu}^{G\{3\}(0)}=\sum_{\alpha \neq \mu, \nu}\left[B_{\alpha \alpha \mu}^{(2)}-B_{\alpha \alpha \nu}^{(2)}+B_{\mu \mu \alpha}^{(2)}-B_{\nu \nu \alpha}^{(2)}\right] .
$$

The 1-loop contributions to the gluonic component are

$$
\begin{aligned}
& \mathcal{T}_{\mu \nu}^{G\{3\}\left(1, N_{c}\right)}=\left\{\mathcal{T}_{\mu \mu}^{G 1}+\mathcal{T}_{\mu \mu}^{G 2}+\mathcal{T}_{\mu \mu}^{G 3}\right\}-(\mu \rightarrow \nu), \\
& \mathcal{T}_{\mu \nu}^{G\{3\}\left(1, \frac{1}{N_{c}}\right)}=\left\{\mathcal{T}_{\mu \mu}^{G 4}+\mathcal{T}_{\mu \mu}^{G 5}\right\}-(\mu \rightarrow \nu), \\
& \mathcal{T}_{\mu \nu}^{G\{3\}\left(1, N_{f}\right)}=\left\{\mathcal{T}_{\mu \mu}^{G 6}+\mathcal{T}_{\mu \mu}^{G 7}\right\}-(\mu \rightarrow \nu) .
\end{aligned}
$$

The tree-level expectation value of $T_{\mu \nu}^{F,\{3\}}$ is

$$
\mathcal{T}_{\mu \nu}^{F\{3\}(0)}=-4\left(F_{\mu \mu}^{(0)}-F_{\nu \nu}^{(0)}\right)
$$

and its derivative with respect to the bare mass is

$$
\frac{\partial \mathcal{T}_{\mu \nu}^{F\{3\}(0)}}{\partial m_{0}}=8\left(F_{\mu \mu}^{(1)}-F_{\nu \nu}^{(1)}\right)
$$

The 1-loop fermion contribution is

$$
\mathcal{T}_{\mu \nu}^{F\{3\}\left(1, N_{f}\right)}=\left\{\mathcal{T}_{\mu \mu}^{F 1}+\mathcal{T}_{\mu \mu}^{F 2}+\mathcal{T}_{\mu \mu}^{F 3}\right\}-(\mu \rightarrow \nu)
$$

\section{I.1 $\mathrm{O}(a)$ improvement}

The 1-loop contributions to the gluonic and fermionic parts due to the improvement are

$$
\mathcal{T}_{\mu \nu}^{G\{3\}\left(1, N_{f}\right)} \longrightarrow \mathcal{T}_{\mu \nu}^{G\{3\}\left(1, N_{f}\right)}+\left\{\mathcal{T}_{\mu \mu}^{G 8}+\mathcal{T}_{\mu \mu}^{G 9}-(\mu \rightarrow \nu)\right\}
$$

and

$$
\mathcal{T}_{\mu \nu}^{F\{3\}\left(1, N_{f}\right)} \longrightarrow \mathcal{T}_{\mu \nu}^{F\{3\}\left(1, N_{f}\right)}+\left\{\mathcal{T}_{\mu \mu}^{F 4}+\mathcal{T}_{\mu \mu}^{F 5}+\mathcal{T}_{\mu \mu}^{F 6}-(\mu \rightarrow \nu)\right\}
$$

respectively. 


\section{J Integrals}

In this appendix we report the definitions of the tree-level integrals which appear in the appendices F, G, H, and I. The functions $\mathrm{c}_{\mu}(p), \mathrm{s}_{\mu}(p), D_{G}(p), D_{F}(p)$ and $\int_{p_{\boldsymbol{\xi}, \theta}}$ are given in appendix E. Repeated indices are not summed over.

Fermionic integrals

$$
\begin{aligned}
F_{\mu \nu}^{(0)} & =\int_{p_{\boldsymbol{\xi}, \theta}} \frac{\bar{p}_{\mu} \bar{p}_{\nu}}{D_{F}(p)} \\
F_{\mu \nu}^{(1)} & =\int_{p_{\boldsymbol{\xi}, \theta}} \frac{m_{0}(p) \bar{p}_{\mu} \bar{p}_{\nu}}{D_{F}^{2}(p)} \\
F_{\mu}^{(2)} & =F_{\mu \mu}^{(0)} \\
F_{\mu}^{(3)} & =\int_{p_{\boldsymbol{\xi}, \theta}} \frac{m_{0}(p) \mathrm{c}_{\mu}(p)}{D_{F}(p)} \\
F_{\mu \nu}^{(4)} & =\int_{p_{\boldsymbol{\xi}, \theta}} \frac{\bar{p}_{\mu} \mathrm{c}_{\nu}(p)}{D_{F}(p)} \\
F_{\mu}^{(5)} & =\int_{p_{\boldsymbol{\xi}, \theta}} \frac{m_{0}(p) \bar{p}_{\mu}}{D_{F}(p)} \\
F_{\mu \nu}^{(6)} & =\int_{p_{\boldsymbol{\xi}, \theta}} \frac{m_{0}(p) \bar{p}_{\mu} \mathrm{c}_{\nu}(p)}{D_{F}^{2}(p)} \\
F_{\mu}^{(7)} & =\int_{p_{\boldsymbol{\xi}, \theta}} \frac{m_{0}^{2}(p) \bar{p}_{\mu}}{D_{F}^{2}(p)} \\
F^{(8)} & =\int_{p_{\boldsymbol{\xi}, \theta}} \frac{m_{0}(p)}{D_{F}(p)}
\end{aligned}
$$

Open Access. This article is distributed under the terms of the Creative Commons Attribution License (CC-BY 4.0), which permits any use, distribution and reproduction in any medium, provided the original author(s) and source are credited.

\section{References}

[1] S. Caracciolo, G. Curci, P. Menotti and A. Pelissetto, The energy momentum tensor for lattice gauge theories, Annals Phys. 197 (1990) 119 [INSPIRE].

[2] S. Caracciolo, G. Curci, P. Menotti and A. Pelissetto, Renormalization of the energy momentum tensor and the trace anomaly in lattice QED, Phys. Lett. B 228 (1989) 375 [INSPIRE].

[3] S. Caracciolo, P. Menotti and A. Pelissetto, Analytic determination at one loop of the energy momentum tensor for lattice QCD, Phys. Lett. B 260 (1991) 401 [InSPIRE].

[4] S. Caracciolo, P. Menotti and A. Pelissetto, One loop analytic computation of the energy momentum tensor for lattice gauge theories, Nucl. Phys. B 375 (1992) 195 [InSPIRE]. 
[5] G. Burgio, S. Caracciolo and A. Pelissetto, Algebraic algorithm for the computation of one loop Feynman diagrams in lattice QCD with Wilson fermions, Nucl. Phys. B 478 (1996) 687 [hep-lat/9607010] [INSPIRE].

[6] S. Capitani and G. Rossi, Deep inelastic scattering in improved lattice QCD. 1. The first moment of structure functions, Nucl. Phys. B 433 (1995) 351 [hep-lat/9401014] [INSPIRE].

[7] S. Capitani, Lattice perturbation theory, Phys. Rept. 382 (2003) 113 [hep-lat/0211036] [INSPIRE].

[8] Y.-B. Yang, M. Glatzmaier, K.-F. Liu and Y. Zhao, The 1-loop correction of the QCD energy momentum tensor with the overlap fermion and HYP smeared Iwasaki gluon, arXiv: 1612.02855 [INSPIRE].

[9] L. Giusti and H.B. Meyer, Thermal momentum distribution from path integrals with shifted boundary conditions, Phys. Rev. Lett. 106 (2011) 131601 [arXiv:1011.2727] [INSPIRE].

[10] L. Giusti and H.B. Meyer, Thermodynamic potentials from shifted boundary conditions: the scalar-field theory case, JHEP 11 (2011) 087 [arXiv:1110.3136] [INSPIRE].

[11] L. Giusti and H.B. Meyer, Implications of Poincaré symmetry for thermal field theories in finite-volume, JHEP 01 (2013) 140 [arXiv:1211.6669] [INSPIRE].

[12] M. Della Morte and L. Giusti, A novel approach for computing glueball masses and matrix elements in Yang-Mills theories on the lattice, JHEP 05 (2011) 056 [arXiv:1012.2562] [INSPIRE].

[13] L. Giusti and M. Pepe, Energy-momentum tensor on the lattice: nonperturbative renormalization in Yang-Mills theory, Phys. Rev. D 91 (2015) 114504 [arXiv:1503.07042] [INSPIRE].

[14] M. Dalla Brida, L. Giusti and M. Pepe, in progress.

[15] B. Sheikholeslami and R. Wohlert, Improved continuum limit lattice action for QCD with Wilson fermions, Nucl. Phys. B 259 (1985) 572 [INSPIRE].

[16] M. Lüscher, Properties and uses of the Wilson flow in lattice QCD, JHEP 08 (2010) 071 [Erratum ibid. 03 (2014) 092] [arXiv:1006.4518] [INSPIRE].

[17] H. Suzuki, Energy-momentum tensor from the Yang-Mills gradient flow, PTEP 2013 (2013) 083B03 [Erratum ibid. 2015 (2015) 079201] [arXiv: 1304.0533] [INSPIRE].

[18] L. Del Debbio, A. Patella and A. Rago, Space-time symmetries and the Yang-Mills gradient flow, JHEP 11 (2013) 212 [arXiv:1306.1173] [InSPIRE].

[19] H. Makino and H. Suzuki, Lattice energy-momentum tensor from the Yang-Mills gradient flow - inclusion of fermion fields, PTEP 2014 (2014) 063B02 [Erratum ibid. 2015 (2015) 079202] [arXiv: 1403.4772] [INSPIRE].

[20] F. Capponi, L. Del Debbio, A. Patella and A. Rago, Renormalization constants of the lattice energy momentum tensor using the gradient flow, PoS (LATTICE2015) 302 (2016) [arXiv: 1512.04374] [INSPIRE].

[21] Y. Taniguchi, S. Ejiri, R. Iwami, K. Kanaya, M. Kitazawa, H. Suzuki et al., Exploring $N_{f}=2+1$ QCD thermodynamics from the gradient flow, Phys. Rev. D 96 (2017) 014509 [Erratum ibid. D 99 (2019) 059904] [arXiv:1609.01417] [INSPIRE]. 
[22] R.V. Harlander, Y. Kluth and F. Lange, The two-loop energy-momentum tensor within the gradient-flow formalism, Eur. Phys. J. C 78 (2018) 944 [Erratum ibid. C 79 (2019) 858] [arXiv: 1808.09837] [INSPIRE].

[23] P. Hasenfratz and F. Karsch, Chemical potential on the lattice, Phys. Lett. B 125 (1983) 308 [INSPIRE].

[24] W.A. Bardeen, A.J. Buras, D.W. Duke and T. Muta, Deep inelastic scattering beyond the leading order in asymptotically free gauge theories, Phys. Rev. D 18 (1978) 3998 [INSPIRE].

[25] S. Capitani, M. Lüscher, R. Sommer and H. Wittig, Non-perturbative quark mass renormalization in quenched lattice QCD, Nucl. Phys. B 544 (1999) 669 [Erratum ibid. B 582 (2000) 762] [hep-lat/9810063] [INSPIRE].

[26] L. Giusti, P. Hernández, M. Laine, P. Weisz and H. Wittig, A strategy to study the role of the charm quark in explaining the $\Delta I=1 / 2$ rule, JHEP 11 (2004) 016 [hep-lat/0407007] [INSPIRE].

[27] L. Giusti and M. Lüscher, Mixing of the $\Delta S=1$ weak interaction hamiltonian, unpublished notes, April 2002.

[28] S.L. Adler, J.C. Collins and A. Duncan, Energy-momentum-tensor trace anomaly in spin 1/2 quantum electrodynamics, Phys. Rev. D 15 (1977) 1712 [INSPIRE].

[29] J.C. Collins, A. Duncan and S.D. Joglekar, Trace and dilatation anomalies in gauge theories, Phys. Rev. D 16 (1977) 438 [inSPIRE].

[30] R. Tarrach, The renormalization of FF, Nucl. Phys. B 196 (1982) 45 [inSPIRE].

[31] M. Lüscher, S. Sint, R. Sommer and P. Weisz, Chiral symmetry and $O(a)$ improvement in lattice QCD, Nucl. Phys. B 478 (1996) 365 [hep-lat/9605038] [INSPIRE].

[32] K. Symanzik, Continuum limit and improved action in lattice theories. 1. Principles and $\phi^{4}$ theory, Nucl. Phys. B 226 (1983) 187 [InSPIRE].

[33] K. Symanzik, Continuum limit and improved action in lattice theories. 2. $O(N)$ Nonlinear $\sigma$-model in perturbation theory, Nucl. Phys. B 226 (1983) 205 [INSPIRE].

[34] M. Lüscher and P. Weisz, On-shell improved lattice gauge theories, Commun. Math. Phys. 97 (1985) 59 [Erratum ibid. 98 (1985) 433] [INSPIRE].

[35] S. Sint and R. Sommer, The running coupling from the QCD Schrödinger functional: a one loop analysis, Nucl. Phys. B 465 (1996) 71 [hep-lat/9508012] [INSPIRE].

[36] T. Bhattacharya, R. Gupta, W. Lee, S.R. Sharpe and J.M.S. Wu, Improved bilinears in lattice QCD with non-degenerate quarks, Phys. Rev. D 73 (2006) 034504 [hep-lat/0511014] [INSPIRE].

[37] J.F. Donoghue and H. Leutwyler, Energy and momentum in chiral theories, Z. Phys. C 52 (1991) 343 [INSPIRE].

[38] H. Panagopoulos and Y. Proestos, The critical hopping parameter in $O(a)$ improved lattice QCD, Phys. Rev. D 65 (2002) 014511 [hep-lat/0108021] [InSPIRE].

[39] M. Lüscher and P. Weisz, Coordinate space methods for the evaluation of Feynman diagrams in lattice field theories, Nucl. Phys. B 445 (1995) 429 [hep-lat/9502017] [INSPIRE].

[40] S. Sint and P. Weisz, Further results on $O(a)$ improved lattice QCD to one loop order of perturbation theory, Nucl. Phys. B 502 (1997) 251 [hep-lat/9704001] [INSPIRE]. 
[41] P. Weisz, Renormalization and lattice artifacts, in Modern perspectives in lattice QCD: quantum field theory and high performance computing. Proceedings, International School, $93^{\text {rd }}$ Session, Les Houches, France, 3-28 August 2009, pg. 93 [arXiv: 1004.3462] [INSPIRE].

[42] R. Frezzotti and G.C. Rossi, Chirally improving Wilson fermions. 1. O(a) improvement, JHEP 08 (2004) 007 [hep-lat/0306014] [INSPIRE].

[43] S. Sint, The chirally rotated Schrödinger functional with Wilson fermions and automatic O(a) improvement, Nucl. Phys. B 847 (2011) 491 [arXiv:1008.4857] [INSPIRE].

[44] M. Dalla Brida, L. Giusti and M. Pepe, QCD in a moving frame: an exploratory study, EPJ Web Conf. 175 (2018) 14012 [arXiv: 1710.09219] [INSPIRE]. 